\title{
1 Ultrafast Structural Changes Decomposed 2 from Serial Crystallographic Data
}

4 Zhong Ren

Department of Chemistry, University of Illinois at Chicago, Chicago, IL 60607, 7 USA

8 Renz Research, Inc., Westmont, IL 60559, USA

10 zren@uic.edu

\section{Summary}

13 Direct imaging of ultrafast and subtle structural events during a biochemical

14 reaction, such as a single electronic transition from one atomic or molecular

15 orbital to another, is highly desirable but has been beyond the reach of protein

16 crystallography. It entails the capability of observing changes in electronic

17 distributions at both an ultrafast time scale and an ultrahigh spatial resolution

18 (Itatani et al., Nature 432, 867, 2004). The recent developments in femtosecond

19 serial crystallography at X-ray free electron lasers (XFELs) have brought the

20 achievable temporal resolution within a striking distance. This paper presents

21 the electron density map decomposed from the XFEL data that shows the

22 remanence of several $3 d$ atomic orbitals of the heme iron at an available spatial

23 resolution although the map component is not an accurate image of the atomic

24 orbitals. A key strategy that has enabled the findings here is a numerical

25 deconvolution to resolve concurrent variations in a series of time-resolved

26 electron density maps so that the electron densities influenced by an electron

27 transfer event can be isolated as a partial change from the overwhelming

28 presence of the bulk electrons that are not directly involved in bonding. Even at

29 the limited spatial resolution, the subtle changes in electron distribution due to a

30 spin crossover can be decoupled from far greater changes due to atomic

31 displacements. Direct observations of electronic orbitals could offer

32 unprecedented mechanistic insights into a myriad of chemical and biochemical 
Ren: Decomposition of ultrafast changes

33 reactions such as electron transfer in redox reactions, and formation, rupture,

34 and isomerization of chemical bonds.

Ligand photodissociation in carbonmonoxy myoglobin $(\mathrm{MbCO})$ has been a benchmark for studying ultrafast protein dynamics in a biological system. A number of studies in time-resolved crystallography have progressively improved the time resolution (from Šrajer et al., Science 274, 1726, 1996 to Barends et al., Science 350, 445, 2015). This paper presents an in-depth analysis of the serial crystallographic datasets of $\mathrm{MbCO}$ that Barends \& Schlichting et al. (2015) contributed to the Protein Data Bank. First, a component of electron density distributions clearly shows the characteristic shape of the high-spin $3 d$ orbitals reappeared at the heme iron upon the photodissociation of the $\mathrm{CO}$ ligand despite the limited accuracy of the orbital image due to the available spatial resolution. Second, the dynamic behaviors of these newly regained $3 d$ orbitals within picoseconds after the photolysis provide long-awaited structural validation for previous spectroscopic observations and computational simulations. Specifically, the newly formed densities are oscillating with the heme iron at a high frequency of a thousand wavenumbers and developing a positional modulation during the first few picoseconds (Champion, Science 310, 980, 2005). The iron pops out of the heme plane at a few picoseconds and recoils back and pops out again afterwards. The dominant oscillation at a low frequency of several tens wavenumbers previously detected by coherence spectroscopy can be clearly resolved from the time series of electron density maps. The associated changes in electron density during the first cycle of the oscillation are largely located in the solvent rather than on the protein or heme, which suggests that the low frequency oscillations in a number of heme proteins, including $\mathrm{MbCO}$, likely originate from a photolysis triggered pressure wave propagating in the solvated protein. Finally, these findings of chemical signals are isolated from coexisting thermal artifacts also by the numerical deconvolution. It is modeled in this study that the ultrashort XFEL pulses cause a transient spike of the local temperature at

63 the heme site of hundreds of $\mathrm{K}$. 
Ren: Decomposition of ultrafast changes

\section{Keywords}

66 ultrafast dynamics, heme protein, X-ray free electron laser, serial crystallography,

67 singular value decomposition

\section{Abstract}

70 Direct visualization of electronic and molecular events during chemical and

71 biochemical reactions will offer unprecedented mechanistic insights. Ultrashort

72 pulses produced by X-ray free electron lasers (XFELs) offer an unprecedented

73 opportunity for direct observations of transient events as short-lived as tens of

74 femtoseconds. This paper presents an in-depth analysis of the serial

75 crystallographic datasets collected by Barends \& Schlichting et al. (Science 350,

$76445,2015)$ that probe the ligand photodissociation in carbonmonoxy myoglobin

77 (MbCO), a long-serving hallmark for observing ultrafast dynamics in a biological

78 system. This analysis reveals electron density changes that are caused directly

79 by the formation of high-spin $3 d$ atomic orbitals of the heme iron upon the $\mathrm{CO}$

80 departure and the dynamic behaviors of these newly formed orbitals in a time

81 series within the first few picoseconds. The heme iron is found vibrating at a

82 high frequency and developing a positional modulation that causes the iron to

83 pop out of and recoil back into the heme plane in succession. These findings

84 here provide long-awaited visual validations for previous works using ultrafast

85 spectroscopy and molecular dynamics simulations. This analysis also extracts

86 electron density variations largely in the solvent during the first period of a low

87 frequency oscillation previously detected by coherence spectroscopy. This work

88 demonstrates the power and importance of the analytical methods in detecting

89 and isolating transient, often very weak signals of electronic changes arising

90 from chemical reactions in proteins.

\section{Introduction}

93 One of the major applications of X-ray free electron lasers (XFELs) is to directly

94 observe ultrafast protein dynamics at atomic resolution during photochemical

95 reactions by serial femtosecond (fs) crystallography (SFX). Time-resolved

96 experiments (Barends et al., 2015; Coquelle et al., 2017; Nango et al., 2016; Nogly

97 et al., 2018; Pande et al., 2016; Tenboer et al., 2014) have been successfully

98 conducted by several groups at XFELs to follow the photodissociation of 
99 carbonmonoxy myoglobin (MbCO; Fig. S1), photoisomerization of the $p$ -

100 coumaric acid chromophore in photoactive yellow protein (PYP), and the

101 hydroxybenzylidene imidazolinone chromophore in a photoswitchable

102 fluorescent protein (Levantino et al., 2015a). While the time resolution, that is,

103 the ability to differentiate transient changes, is limited to tens of fs determined by

104 the duration of the XFEL pulses, the reported time resolution for MbCO and PYP

105 was around hundreds of fs due to some timing jitter between a short laser pump

106 and an XFEL probe.

It is well established that a ligand bound to the heme iron helps to split its

110 and the doubly degenerate $e_{\mathrm{g}}$ at a higher energy, so that the six $3 d$ electrons of

$111 \mathrm{Fe}(\mathrm{II})$ form three pairs occupying the lower energy orbitals, thus the net spin is

112 zero. Upon photodissociation, the CO ligand parks at a nearby docking site,

113 while the heme iron moves out of the heme plane towards the proximal side.

114 The absence of a bound ligand returns the five degenerate $3 d$ orbitals of the iron

115 back to approximately identical energy levels so that they are all occupied

116 resulting in a high-spin, deoxy iron. As the porphyrin structure becomes

117 buckled, several side chains and helices of the globin move accordingly (Fig. 1).

118 These light-induced structural changes are highly reproducible and have been

119 observed by both static and time-resolved crystallography, repeatedly (Bourgeois

120 et al., 2003, 2006; Chu et al., 2000; Kachalova et al., 1999; Schotte et al., 2003; Šrajer

121 et al., 1996, 2001). Recently, they have been captured once again at a sub-

122 picosecond (sub-ps) time scale in MbCO crystals using XFEL pulses (Barends et

123 al., 2015), a major achievement in SFX. However, no significantly new structural

124 finding is beyond those already obtained by time-resolved Laue diffraction at

125 synchrotrons (Bourgeois et al., 2007; Clifton et al., 1997; Ren et al., 1999), which is

126 one thousand times slower at a time resolution of 100 ps limited by the bunch

127 length of the synchrotrons (Aranda et al., 2006; Bourgeois et al., 2006; Jung et al.,

128 2013; Ren et al., 2012; Schotte et al., 2012).

130 The signals of ultrafast structural events could be too weak to be observed 131 clearly by the standard crystallographic analyses. In this work, I reexamine the 132 time-resolved XFEL datasets of MbCO deposited in the Protein Data Bank (PDB; 
$1335 \mathrm{cmv}, 5 \mathrm{cn} 4-9$, and 5cnb-g) by Barends \& Schlichting et al. (2015) with a

134 previously developed analytical method based on singular value decomposition

135 (SVD; Ren, 2013a; Ren et al., 2012, 2013; Schmidt et al., 2003). This SVD analysis

136 unravels several ultrafast events that have been identified by spectroscopy and

137 computational studies but have not been directly observed as images of electron

138 density distribution. The new findings along with several previously known

139 structural events upon photolysis of $\mathrm{MbCO}$ are summarized in a Gantt chart

140 (Fig. 1).

\section{Heterogeneous signals in dynamic crystallography}

143 Compared to the well-established structural events of MbCO photolysis such as

144 ligand docking and heme doming, the ultrafast signals are small in amplitude

145 and low in population. As a result, they do not stand out at a given time point,

146 thus went unnoticed in the conventional analyses. As demonstrated below, these

147 weak yet consistent signals can be manifested as major components resulting

148 from SVD when multiple datasets collected at different time points are jointly

149 examined. This necessity for decomposition presents both a challenge and a

150 unique opportunity for dynamic crystallography, broadly defined as

151 experimental and analytical approaches that tightly join crystallographic datasets

152 and their metadata (Ren et al., 2013). We have developed a numerical process of

153 deconvolution, which is able to identify and extract the dynamic signals from a

154 large collection of datasets acquired at varying experimental conditions such as

155 time points and temperatures (Bandara et al., 2017; Kim et al., 2017; Ren, 2016;

156 Ren et al., 2012; Yang et al., 2011; Zeng et al., 2015). In essence, this strategy takes

157 advantage of the structural heterogeneity, rather than preemptively avoiding

158 them as in the common practice of static protein crystallography. In a nutshell,

159 dynamic crystallography consists of two major steps: first, to experimentally

160 introduce a functionally-relevant structural heterogeneity; and second, to

161 numerically resolve the heterogeneous conformational species as long as their

162 compositions vary as a function of an experimental parameter (Ren et al., 2013).

163 Here, time-resolved serial crystallography, with the variable being the ultrashort

164 time delay, is simply a special case in the broader scope of dynamic

165 crystallography. Therefore, the previously established methodology is largely

166 applicable (Methods). 


\section{Ultrafast dynamics during photodissociation of $\mathrm{MbCO}$}

169 The departure of the CO ligand from the iron porphyrin system has been said 170 within the first 15-20 fs upon photolysis (Dunietz et al., 2003; Falahati et al., 2017, 171 2018). This signal is clear in the earliest time point of $\sim 30$ fs (nominally -0.1 ps;

172 Fig. S2). However, the photolyzed CO ligand is already observed in its docking

173 site 3.5-4.5 $\AA$ away from the heme iron at tens to hundreds of fs (Figs. 2c and

174 3agj) unlike recently suggested by quantum wavepacket dynamics that the 175 ligand departs only $0.4 \pm 0.1 \AA$ (Falahati et al., 2018). A transient charge transfer 176 from the iron to porphyrin subsequently taking place during 50-300 fs has been 177 identified as a key event by resonance Raman spectra (Franzen et al., 2001). A 178 high-spin iron is resulting in the charge transfer from the porphyrin back to the 179 iron. The transient charge transfer state is not visible in the maps calculated from 180 the datasets of Barends \& Schlichting et al. perhaps due to a broad distribution of 181 the transferred electron over the porphyrin. However, the larger, high-spin iron 182 is clearly manifested in the earliest maps (Figs. 2, 3, and S2). Vibrational signals

183 have been observed by ultrafast spectroscopy, which is attributed to a

184 discontinuity or "seam" at the intersection of two energy landscapes of the

185 photoexcited $\mathrm{MbCO}$ and the ligand dissociated product (Champion, 2005).

186 Dissipation of such vibrational energy in the protein matrix and its solvent

187 environment consists of at least two distinct components: localized, high

188 frequency vibration modes of the heme at approximately one thousand

189 wavenumbers; and global, low frequency oscillations of the protein at several

190 tens wavenumbers (Fujisaki and Straub, 2005). This analysis provides direct

191 visual evidences to validate both components and to delineate the low frequency

192 oscillations in greater details (Fig. 7).

194 The most observed high frequency modes range from hundreds to more than 195 a thousand wavenumbers (Armstrong et al., 2003; Kitagawa et al., 2002;

196 Mizutani, 1997). The corresponding vibrational periods of these high frequency

197 modes of 24-50 fs are comparable to the XFEL pulse duration. Hence an

198 individual cycle of such vibrations is beyond what can be traced out by the 199 current XFEL datasets. Nevertheless, the averaged effect of these high frequency 200 vibrations is detectable (Figs. 2 and 3) in the XFEL datasets of Barends \& 
201 Schlichting et al. Amplitude modulation and damping of the high frequency

202 mode $v$ was simulated with a period of $350 \mathrm{fs}$ and a time constant of $1.3 \mathrm{ps}$

203 (Barends et al., 2015). There are not sufficient time points in the existing datasets

204 to determine these amplitude modulation and damping. However, a slower

205 modulation of the high frequency modes is clearly present in these datasets (Figs.

2062 and S4; Movie S1). These in-plane modes are thought to be coupled to out-of-

207 plane motions of the heme and its iron (Nagy et al., 2005), which take place

208 within a fraction of ps and continue to develop non-exponentially for another

209 hundreds of ps according to molecular dynamics simulations (Henry et al., 1985;

210 Kuczera et al., 1993). Recent simulations (Barends et al., 2015; Falahati et al.,

211 2017) suggested that many transient species, including those with iron in or out

212 of the plane, are mixed together before 1 ps. It was also suspected that the iron

213 may recoil back into the heme plane after its initial drop out of the plane (Gruia

214 et al., 2008). This analysis of the XFEL datasets finds that the out-of-plane

215 displacement of the iron takes place simultaneously with an in-plane shift of the

216 porphyrin (Movie S3). There is clear visual evidence to show that the first out-of-

217 plane move peaks at a few ps and that the recoil and the second drop take place

218 afterwards (Figs. 2cf and S4c; Movie S1). The signal associated with the iron

219 displacement lingers on for many $\mu$ s before the ligand rebinding as captured by

220 the time-resolved Laue diffraction (Šrajer et al., 2001).

At a low frequency region, the dominant oscillations range between 25 and 45

$223 \mathrm{~cm}^{-1}$, corresponding to a period around 1 ps (Gruia et al., 2008; Rosca et al., 2002).

224 Such a low frequency oscillation is within the reach of the sub-ps time resolution

225 achieved with XFEL pulses. Therefore, each individual cycle of the low

226 frequency oscillation should be, and indeed is, visible in the datasets of Barends

227 \& Schlichting et al. Here I demonstrate that such an oscillation occurs more in

228 the solvent channels than in the protein or the heme at a low frequency

229 comparable to those detected by spectroscopy (Fig. 7). This analysis supplies the

230 long missing visual evidence to support such a low-frequency oscillation in a

231 solvated protein.

232

233 Imaging the geometric shapes of atomic orbitals

234 The shape of a $d$ orbital of copper was first experimentally imaged by Zuo et al. 
235 in their difference electron density map (Humphreys, 1999; Zuo et al., 1999),

236 which is not, however, without controversy (Mulder, 2011; Scerri, 2000).

237 Nevertheless, experimenters continued to draw parallels between captured

238 images and atomic or molecular orbitals, sometimes with the analytical power of

239 digital reconstructions. Molecular orbital tomography constructs images of

240 highest occupied molecular orbital from many projections of aligned molecules

241 (Itatani et al., 2004; Ogilvie, 2011; Schwarz, 2006; Stapelfeldt, 2004; Vozzi et al.,

242 2011). Both the highest occupied and lowest unoccupied molecular orbitals of

243 flat molecules, such as pentacene, were scanned using scanning tunneling

244 microscopy (Chaika, 2014; Patera et al., 2019; Repp et al., 2005). Field-emission

245 electron microscopy and angle-resolved photoemission spectroscopy can directly

246 capture or indirectly reconstruct orbital images or transformation of orbital

247 configurations (Mikhailovskij et al., 2009; Puschnig et al., 2009). More recently, a

248 photoionization experiment showed the nodal structures of the atomic orbital of

249 excited hydrogen atoms (Stodolna et al., 2013). A charge-density analysis at an

250 ultrahigh resolution of $0.48 \AA$ showed iron $3 d$ and sulfur $3 p$ densities (Hirano et

251 al., 2016). In all these achievements, the experimentally captured electron

252 densities with their unique shapes are directly influenced by electrons occupying

253 specific orbitals. Therefore, in this paper, the images and motion pictures of

254 difference electron densities with their characteristic nonspherical shape are

255 attributed to a superposition of several atomic orbitals and their dynamics.

256 However, this is not to say that the accurate image of an atomic orbital has been

257 captured, which is not a proper task for protein crystallography, given the limit

258 in spatial and temporal resolutions.

260 Results

261 Decomposition of difference maps and simulated annealing omit maps

262 To analyze the time-resolved serial crystallographic data of horse heart (hh)

$263 \mathrm{MbCO}$, one dark and 12 light datasets are obtained from PDB (Fig. 1; Barends et

264 al., 2015), among which three light datasets collected at the shortest time delays

265 of 0 and \pm 0.1 ps span both sides of time 0 that is centered on the excitation laser

266 flash of $150 \mathrm{fs}$. These datasets are expected to capture ultrafast signals at delay

267 times as short as a few fs to tens of fs. However, these ultrafast signals are also

268 inevitably mixed with signals at longer delays as far as the X-ray pulses stretch 
269 (Haldrup et al., 2011). These sub-ps time points were taken at the same

270 controlled time delay of 0.5 ps. But the diffraction images were binned according

271 to the timestamp records from an experimental timing tool (Bionta et al., 2011,

272 2014), which produced a number of short delays before 1 ps. Several longer time

273 points ranging from 3 to 150 ps are also included in this analysis (Fig. 1) along

274 with a deoxy $\mathrm{hhMb}$ dataset collected from a synchrotron beamline at $7^{\circ} \mathrm{C}$, which

275 represents the end product of the $\mathrm{MbCO}$ photolysis, and two hhMb structures

276 (1dws/t) in the photolyzed state captured by cryo-trapping (Chu et al., 2000).

Two types of electron density maps, namely difference Fourier map and simulated annealing omit map (SAOM), are produced and subjected to the SVD analyses separately. Each type carries its own advantage (Methods). While difference Fourier maps are sensitive for detecting weak signals, they are also highly susceptible to systematic errors that often overwhelm the signal. It is critically important to choose a proper reference dataset, which is preferably obtained under the identical experimental condition, if not from the very same crystal as the light dataset. An alternative is to use SAOMs, where a small portion of a protein structure is set aside from structure refinement using the simulated annealing algorithm. In such omit maps, the electron densities within the omitted region shall not be biased by the protein model, thus are considered as the most authentic representation of the experimentally observed electron densities (Hodel et al., 1992). A collection of SAOMs within a common omitted region, although less sensitive to subtle changes compared to difference maps, permits a joint analysis of maps derived under very different experimental conditions, which is usually difficult with difference maps.

Difference Fourier maps are synthesized using a Fourier coefficient set of

297 the structure factor amplitudes from a dark dataset $(5 \mathrm{cmv})$, it is important that a

298 light dataset at a given time point is also used as a reference (Table S1) to reveal

299 changes with respect to that specific time point (Methods). Therefore, a

300 collection of difference maps produced with the same reference dataset

301 constitutes a time series that show continuous changes from the reference time

302 point and on. For example, $-F_{0}$ series refers to the difference maps with the 
303 reference dataset at the nominal 0 ps. For omit maps, the heme and several side

304 chains within its immediate vicinity are omitted to produce SAOMs of the ligand

305 binding site (Methods). A few static structures determined at room temperature

306 of sperm whale (sw) Mb in the aquomet (1bz6), deoxy (1bzp), and CO-bound

307 (1bzr) forms are also included (Kachalova et al., 1999), along with three hhMb

308 structures (1dwr/s/t) (Chu et al., 2000) as control data points in this joint analysis.

309 The resolutions of these static datasets are truncated to $1.75 \AA$ to match the XFEL

310 datasets.

Altogether, a total of 93 difference maps (Table S1 and Fig. S2) and 20 omit maps are obtained for two independent SVD analyses. The electron densities are masked around the heme site unless stated otherwise (Methods). In this work, the significant signals are identified by the top nine components from SVD (Fig. 2e). A numerical procedure called rotation in the SVD space plays an important role to extract chemically sensible components from these (difference) electron density maps (Henry and Hofrichter, 1992; Ren, 2013a, 2013b, 2016). Proper SVD rotations reveal the correlation between the core data, i.e., electron densities, and their metadata, i.e., delay time, sample temperature, and other experimental parameters (Methods). Given their highly dynamic nature, the ultrafast signals due to multiple causes are often entangled before suitable rotations are found in the multi-dimensional SVD space (Methods). Using this decomposition and

324 rotation strategy, I identify signals that reveal the electronic redistributions

325 between high and low spin of the iron orbitals at the available spatial resolution.

\section{Molecular image of low-spin to high-spin crossover}

328 The ultrafast signals within several fs to a few tens of fs are manifested in the

329 fourth component, denoted as $\boldsymbol{U}_{4}$, of 93 difference maps. The component $\boldsymbol{U}_{4}$

330 exists only in the map series with the dark dataset as reference (red, $-F_{\text {dark }}$ series

331 in Fig. 2). That is to say, all signals in this component have occurred by the time

332 of the very first time point at $\sim 30$ fs (nominally $-0.1 \mathrm{ps}$ ), therefore the map

333 series $-F_{-0.1}$ shows the cancellation of this component (orange series in Fig. 2).

334 Some of these signals start to decay slightly before 1 ps and diminish

335 significantly after 3 ps (Fig. 2cd). The corresponding map of $\boldsymbol{U}_{4}$ reveals the

336 previously well-established events arising from the CO docking, out-of-plane 
337 motion of the iron, and an uneven heme doming upon photolysis of MbCO (Fig.

338 3). The negative densities associated with the pyrrole rings and their propionic

339 acid side chains increase in intensity from ring $A$ to $B$ to $C$ to D (Fig. 3c). Positive

340 densities are found at a cross section $1.1 \AA$ above the heme plane towards the

341 distal side (Fig. 3b).

More remarkably, $\boldsymbol{U}_{4}$ captures some hitherto unseen signals characterized by a cubic-shaped network of positive densities that cage the iron atom. To facilitate a more concise description, a cross section of $Z=0$ is defined as the heme plane and the cross section of $X=0$ is the dividing plane between two propionic acid side chains, from which the third orthogonal cross section of $Y=0$ can be derived (Fig. S1). The eight corners of the positive cube are oriented along the $X$ and $Y$ cross sections. Strong positive densities appear in the middle of four six-membered rings encircled by adjacent pyrroles, the connecting methane bridge, and the heme iron (marked 1-4 in Fig. 3c). These positive features reach their four minima when they cross the heme plane. These positive densities become more prominent above and below the heme plane (Fig. 3). The eight corners of this cube are located at $1.7 \pm 0.2 \AA$ away from the iron. This cubicshaped network is asymmetric with a notable skew towards CD rings (Fig. 3fh). Meanwhile, the iron skews in the opposite direction towards the AB rings with an out-of-plane displacement towards the proximal side (Fig. 3e). But the major features of this cube seem more symmetric in the $Y$ cross section.

Similar features are also evident in the seventh component $\boldsymbol{U}_{7}$ from an

362 after a proper rotation (Methods) represents the reaction coordinate of the ligand 363 dissociation. $\mathrm{Mb}$ structures along this reaction coordinate form a lineup on $\mathrm{c}_{7}$

364 dimension regardless of sample temperatures: from the ligated structures of

$365 \mathrm{MbCO}$, to the time-resolved structures, to several photoexcited species including 366 the static structures $\mathrm{Mb}^{*} \mathrm{CO}$ and ${ }^{* *} \mathrm{CO}$, eventually to deoxy $\mathrm{Mb}$ (Fig. 4e). $\boldsymbol{U}_{7}$ of 367 the omit maps contains the usual signals of ligand dissociation, such as strong 368 negative densities on the ligand indicating dissociation and a positive layer on 369 the distal side of the heme indicating its doming (Fig. 5), except that the quality 370 of these signals are not as good as those from difference maps because the 
371 SAOMs are derived from datasets in very different experiments. Despite the

372 systematic errors, the seventh component $\boldsymbol{U}_{7}$ of the omit maps again clearly

373 shows the cubic network of positive densities around the iron. This cubic

374 network also skews towards the CD rings of the heme. This is a reoccurrence of

375 the same signals shown in $\boldsymbol{U}_{4}$ of the difference maps (compare Figs. 3 and 5). The

376 eight corners of the positive cube are $1.9 \pm 0.2 \AA$ away from the iron.

It is clear that the positive densities arranged in the cubic network around the iron, either in the difference maps or in the omit maps, cannot be easily attributed to accidental noise or systematic errors such as thermal effect or Fourier truncation error (see Discussion). They originate from the intrinsic

382 signals induced by light and coexist with the previously seen signals of ligand

383 dissociation. While multiple interpretations of these distinct cube-shaped signals

384 may apply, this positive network clearly indicates a larger, high-spin iron as an

385 immediate overall effect upon photolysis. Here I further attribute the distinct

386 shape of the positive feature to the instantaneous regaining of the high-spin $3 d$

387 orbitals of the heme iron. However, the observed cubic densities here are not an

388 accurate image of a probability distribution of a $3 d$ electron. They are resulted

389 from a net gain of electron densities as a fully ligated iron transitions to the high-

390 spin deoxy state when several $3 d$ orbitals are reoccupied by unpaired electrons.

391 Calculations of the combined probability distributions of the textbook $3 d_{x z}$ and

$3923 d_{y z}$ with either $3 d_{x y}$ or $3 d_{x^{2}-y^{2}}$ orbitals show features highly similar to the

393 observed cubic-shaped map (Fig. 31m). The strongest electron densities are

394 located at eight corners of the cube where the lobes of $3 d$ orbitals intercept. It is

395 entirely possible that the observed cubic densities result from not only a

396 superposition of several $3 d$ orbitals but also an average of multiple electronic

397 configurations. See Discussion below. Such orbital changes have not been

398 captured previously in protein crystals by X-ray crystallography.

However, the loss of the paired low-spin electrons in the ligated state is not 401 clear in the negative densities. Presumably, the loss of the CO ligand and the 402 drop of the iron out of the heme plane cause the displacement of many more 403 electrons than the low-spin electrons. Negative densities in the component $\boldsymbol{U}_{4}$ 
Ren: Decomposition of ultrafast changes

404 are overwhelming. At the available spatial resolution, the loss of the low-spin

405 electrons is not separated from the main events upon photolysis.

\section{Development, modulation, and damping after 1 ps}

If the cubic network of positive densities is indeed influenced by the newly formed $3 d$ orbitals, the dynamic behaviors of these high-spin electrons should be visible in the time points shortly after the initial formation. The observed evolution of the cubic network provides the necessary validation. Along the dimension $c_{1}$ in the SVD space of 93 difference maps, all time points after 1 ps, including the deoxy $\mathrm{Mb}(5 \mathrm{~d} 5 \mathrm{r}$, representing a time point of $\infty)$, are well separated from the sub-ps time points around $c_{1}=0$ (Fig. 2ac). Therefore, the first component $\boldsymbol{U}_{1}$ of the difference maps indicates a major development after 1 ps and represents more permanent changes afterwards. The cubic positive densities in $\boldsymbol{U}_{4}$ have no sign in $\boldsymbol{U}_{1}$. Among the major features captured by $\boldsymbol{U}_{1}$ (Fig. S3), the most noticeable is a rather skewed displacement of the iron towards the $A B$ rings of the heme. In the meanwhile, the motion of His93 corroborates the skewed displacement of the iron (Fig. S3ae). It is also shown below that the entire heme 
438 moves in its plane towards the $\mathrm{AB}$ rings as well. Such long-lived signals in $\boldsymbol{U}_{1}$

439 from 1 ps and on are in good agreement with a wide distribution of individual

440 trajectories and interquartile range of iron displacement predicted by molecular

441 dynamics simulations (Barends et al., 2015; Henry et al., 1985; Kuczera et al.,

442 1993). However, the cryo-trapped structures, $\mathrm{Mb}{ }^{*} \mathrm{CO}$ and ${ }^{* *} \mathrm{CO}$, are two

443 exceptions. They exhibit very little characteristics in $\boldsymbol{U}_{1}$ (Fig. 2ac), even though

444 they presumably represent long time delays. It can be reasoned that the skewed

445 displacement of iron requires concerted motions in the proximal His93 and helix

446 F, which are evidently forbidden at cryogenic temperatures.

Both $\boldsymbol{U}_{1}$ and $\boldsymbol{U}_{4}$ feature the signal of the iron out-of-plane displacement (Figs. $3 e$ and S3c). Therefore, the large positive coefficients $c_{1}$ and $c_{4}$ occurring simultaneously around several ps indicate that the iron pops out of the heme plane most at this time but only momentarily. The later decay of the fourth component $\boldsymbol{U}_{4}$ records a recoiling of the iron back into the heme plane around $100 \mathrm{ps}$ (Movie S1). Another major signal revealed after $1 \mathrm{ps}$ is a modulation of the new formation of $3 d$ orbitals indicated by the cubic-shaped positive densities around the iron. This is a positional modulation manifested in the sixth and eighth components of the difference maps. While the corresponding coefficients $c_{6}$ and $c_{8}$ are nearly constant in the sub-ps range, they start to ramp up and vary at a few ps. This variation can be most easily modeled as a two-dimensional oscillation at a period of $220 \pm 20$ ps given the limited time points (Fig. 2f).

Additional observations in the future may update this positional oscillation. The $\boldsymbol{U}_{6}$ component features a distorted network of positive densities around the heme iron (Fig. S4) similar to $\boldsymbol{U}_{4}$ but with an inclination towards ring B. In $\boldsymbol{U}_{6}$, the strong negative density on iron is skewed towards the proximal side in contrast to $\boldsymbol{U}_{4}$ (compare Figs. 3e and S4c). The positive peak on the proximal side of the iron is also extremely skewed (Fig. S4c). In a linear combination sense, $\boldsymbol{U}_{6}$ and $\boldsymbol{U}_{8}$ can be interpreted as variations that are combined into $\boldsymbol{U}_{4}$ resulting in an oscillation of a period at a few hundreds of ps (Fig. 2f). Specifically, the cubicshaped densities lean towards ring D from 0.5 to 3 ps, return to the mean position after 50 ps, then sway towards ring $B$ in the opposite direction (Fig. $2 \mathrm{f}$ and Movie S1). Near the end of the first oscillation, the iron again pops out of the heme plane for the second time and severely skews towards $A B$ rings (Movie 
472 S1). These observations of the iron motions support the previous model of iron 473 recoiling (Gruia et al., 2008). Such oscillating behavior suggests modulation and

474 damping of the vibrational modes. In other words, the cubic network of positive

475 densities is not only observed in the earliest time points. The motions of this

476 network are captured in later time points as well. This 220-ps oscillation is

477 largely along BD cross section (Fig. S1), while damping of the vibrational

478 amplitude was only recorded up to 150 ps thus insufficient to depict a complete

479 picture. Two other more significant components $\boldsymbol{U}_{2}$ and $\boldsymbol{U}_{3}$ also show an

480 oscillation of a distinct type as discussed below (Fig. 2b).

\section{Vibration induced by XFEL pulses and instantaneous local temperature jump}

483 An independent SVD analysis of 20 simulated annealing omit maps (SAOMs)

484 masked around the heme site (Methods) offers a direct comparison of the XFEL

485 data with previous synchrotron data. Among the top eight components of the

486 SAOMs, the first component reveals a well-defined molecular image of the heme

487 and its surrounding (Fig. S5); and the second and fourth components apparently

488 account for differences between $\mathrm{hhMb}$ and $\mathrm{swMb}$. It is the third component $\boldsymbol{U}_{3}$

489 that clearly distinguishes the XFEL datasets from the synchrotron datasets in a

490 temperature-dependent manner (Fig. 4ace). In other words, the cryo structures

491 (1dwr-t), ambient temperature structures $(1 \mathrm{bz} 6 / \mathrm{p} / \mathrm{r})$ including the deoxy hhMb at

$4927^{\circ} \mathrm{C}(5 \mathrm{~d} 5 \mathrm{r})$, and the room temperature XFEL structures line up in an ascending

493 order along the dimension $c_{3}$. Not incidentally, the photolyzed structures

$494 \mathrm{Mb}^{*} \mathrm{CO}$ and ${ }^{* *} \mathrm{CO}$ have the most negative coefficient $c_{3}$ because they were

495 obtained at temperatures even lower than $100 \mathrm{~K}$ (Chu et al., 2000). The

496 corresponding decomposed map $\boldsymbol{U}_{3}$ reveals the thermal effects in electron

497 densities of the heme as a nearly symmetric shell of spherical positive densities

498 around the iron with very little positive or negative density on the iron itself (Fig.

499 6), which transitions into a square shape with four corners centered at the six-

500 membered rings in the heme plane (marked 1-4 in Fig. 6e). This symmetric $\boldsymbol{U}_{3}$ of

501 the omit maps shows no usual signals for ligand dissociation and heme doming

502 therefore contrasts with the asymmetric $\boldsymbol{U}_{4}$ of the difference maps characterized

503 by the distinct cube-shaped positive density maxima at the eight corners. Two

504 sheets of positive densities are found at the cross sections parallel to, and $1.3 \AA$

505 away from, the heme plane on both sides (Fig. 6df). They are connected via 
506 pillar-shaped positive densities that run through the six-membered rings

507 (marked 1-4 in Fig. 6e) and the pyrrole rings (marked 5-8 in Fig. 6e) along with

508 some other positive densities outside the heme (marked 9-20 in Fig. 6e). It is

509 noteworthy that such thermal effects do not stand out as a major component in

510 the SVD analysis of difference maps because they are largely self-canceled

511 during the subtraction of the reference.

The temperature dependency and the symmetry of the signals in $\boldsymbol{U}_{3}$ strongly suggest that these features are related to the local temperature at which the $\mathrm{MbCO}$ molecules were probed, rather than artifacts arising from structural refinement or difference in spatial resolutions among datasets. All datasets used for the analysis are unified to a similar spatial resolution (Methods). The positional parameters of the models within the omitted region and the thermal parameters of the entire globin are intentionally "forgotten" in the calculation of the omit maps. The large $c_{3}$ coefficients of the XFEL datasets are due to instantaneous temperature elevation at the heme site upon exposure to each ultrashort XFEL pulse, which introduces isotropic vibrations at high frequencies to the entire tetrapyrrole cofactor as captured by $\boldsymbol{U}_{3}$. This molecular image of $\boldsymbol{U}_{3}$ shows an increasing intensity from cryo to ambient temperatures and to a very high local temperature induced by XFEL pulses (Movie S2). Solely based on an estimate by linear extrapolation without other validations, the instantaneous temperature of the heme may exceed $500 \mathrm{~K}$ during an 80-fs XFEL pulse (Fig. 6i). However, it remains to be seen whether this analysis of the omit maps restricted within the heme site can be extended to show that the temperature of the globin or the crystals has a significant elevation during an XFEL pulse.

Identification of such symmetric and temperature-dependent signals is important for interpretation of the highly dynamic ultrafast signals. The fact that the symmetric $\boldsymbol{U}_{3}$ and the asymmetric $\boldsymbol{U}_{7}$ of the omit maps are orthogonal to each other mathematically guarantees that these coexisting components are not interchangeable and not cross contaminated between them (Methods). The cubic shaped $\boldsymbol{U}_{7}$ of the omit maps and its counterpart $\boldsymbol{U}_{4}$ of the difference maps are the chemical consequence of ligand dissociation; the symmetrical and spherical shaped $U_{3}$ originates from the thermal consequence. One cannot compensate for 
540 the other and both coexist in all maps. The equivalents can be stated: The cubic 541 positive densities influenced by the $3 d$ orbitals are not due to thermal vibrations;

542 the symmetrically enlarged iron and the thickened heme due to an instantaneous

543 temperature elevation have been isolated from the chemically relevant signals. It

544 is worth noting that the ultrafast onset of $\boldsymbol{U}_{4}$ of the difference maps (Fig. 2cd) is

545 not in contradiction with $\boldsymbol{U}_{7}$ of the omit maps contained not only in time-

546 resolved XFEL datasets but also in the static structures of deoxy $\mathrm{Mb}$ and

547 photolyzed $\mathrm{Mb}^{*} \mathrm{CO}$ and ${ }^{* *} \mathrm{CO}$ at different temperatures (Fig. 4e). Instead,

548 regaining of the high-spin $3 d$ orbitals of the iron occurs before the first time point

549 at tens of fs; this permanent change lasts throughout the dissociation reaction

550 into the deoxy product.

551

552

553

The asymmetric $\boldsymbol{U}_{7}$ of the omit maps also describes an important but unfamiliar structural event upon the ligand dissociation - the heme in-plane movement. This type of motion is harder to capture in difference maps due to little net gain or loss of electron density that would be caused by an in-plane movement of the heme. With respect to the protein framework, the heme moves towards its $\mathrm{AB}$ rings in addition to the other changes (Movie S3). Heme in-plane sliding is consistent with the strong signals of the skewed iron displacement and the proximal His93 (Fig. S3) that are closely related to structural responses in the globin. Similar in-plane sliding is even more significant in tetrameric hemoglobins (Baldwin and Chothia, 1979), which is the origin of the cooperative oxygen affinity (Ren, 2013b). However, the functional role of the heme in-plane

564 here on ultrafast structural changes.

\section{Global oscillation at low frequency}

567 A recent solution scattering experiment using XFEL pulses showed that the

568 radius of gyration $R_{\mathrm{g}}$ first increases within 1 ps upon photodissociation of $\mathrm{MbCO}$ 569 and then oscillates with a period of $3.6 \mathrm{ps}$, equivalent to $9 \mathrm{~cm}^{-1}$, a frequency lower 570 than those observed by coherence spectroscopy (Levantino et al., 2015b). The 571 molecular volume increases afterward with a phase shift. These observations 572 indicate a wavelike motion propagating outward in the protein. Recent 573 molecular dynamics simulations have confirmed that a pressure wave generated 
Ren: Decomposition of ultrafast changes

574 by photolysis reaches the molecular surface at 300 fs along the direction

575 perpendicular to the heme plane (Brinkmann and Hub, 2016), which corresponds

576 to a frequency of $28 \mathrm{~cm}^{-1}$ if the wave is reflected by the surface and oscillates. It

577 was found by the simulations that the oscillation in solvent would be

578 overdamped thus dissipates quickly.

Some SVD components derived here display strict correlation suggesting

582 is evidently equivalent to the $\boldsymbol{U}_{5}-\boldsymbol{U}_{8}$ pair of the omit maps (Fig. $4 \mathrm{~b}$ ). When the

583 difference maps of the entire globin are analyzed, the same signals emerge as the

584 top-ranked $\boldsymbol{U}_{1}-\boldsymbol{U}_{3}$ pair suggesting that the local signals at the heme site concur

585 with global signals over the entire globin (Fig. 7). Interestingly, this pair of

586 components displays a near-perfect circular correlation at sub-ps delays but is

587 almost absent at longer delays $>1$ ps (Fig. 7afgh). Such an oscillation at a low

588 frequency requires at least two orthogonal components to depict. The oscillating

589 signals have spread over the entire globin at sub-ps delays while their

590 amplitudes decay to nearly zero after a few ps. Based on least-squares fittings of

591 the corresponding coefficients (Methods), the frequency of the oscillation is

592 determined to be $36 \pm 1 \mathrm{~cm}^{-1}$, corresponding to a period of $0.93 \mathrm{ps}$. The equivalent

593 oscillation frequencies determined from the difference maps and omit maps at

594 the heme site are $42 \pm 2 \mathrm{~cm}^{-1}$ (a period of $0.8 \mathrm{ps}$ ) and $37 \pm 1 \mathrm{~cm}^{-1}$ (a period of $0.89 \mathrm{ps}$ ),

595 respectively.

The low frequency oscillation determined from the XFEL datasets of Barends

599 coherence spectroscopy (Gruia et al., 2008; Rosca et al., 2002). I postulate that this

600 low frequency reflects the molecular size of the solvated $\mathrm{Mb}$ rather than the

601 property of the heme. First, the corresponding period of 0.93 ps is about the time

602 that a mechanical wave takes to traverse $14 \AA$, that is, an average distance from

603 the heme to the molecular surface of $\mathrm{Mb}$, given a speed of sound at $1.5 \mathrm{~km} / \mathrm{s}$ or

$604 \mathrm{~nm} / \mathrm{ps}$ (Fig. S1). Second, the molecular sizes of several heme proteins, such as

605 cytochrome c (12 kDa), Mb (17 kDa), and cystathionine $\beta$-synthase (63 kDa),

606 show good anticorrelation with their dominant low frequencies of 44, 40, and 25

$607 \mathrm{~cm}^{-1}$, respectively (Gruia et al., 2008; Karunakaran et al., 2010, 2014). Third, no 
608 association between the oscillating signals and specific structural features can be

609 found, such as the heme, ligand, surrounding side chains, or helices (Fig. 7ik).

610 These oscillating signals sharply contrast with strong, non-oscillating signals

611 associated with the heme and various helices evidenced by the second and fourth

612 components of the difference maps (Fig. 7jl), where helices E, G, and H move

613 away from the heme in response to ligand dissociation (Barends et al., 2015).

614 These motion signals of various structural elements are much enhanced by SVD.

The oscillating signals at low frequency seem to propagate via solvent within the protein matrix rather than the globin. Strong positive and negative peaks in $\boldsymbol{U}_{5}, \boldsymbol{U}_{6}$, and $\boldsymbol{U}_{8}$ of the omit maps are consistently found in the solvent region away from the heme and protein. However, a specific description on how the solvent responses to ligand dissociation is difficult, since the solvent structure is far less

$622 \boldsymbol{U}_{8}$ can be judged by a sum of the absolute values $\left|\boldsymbol{U}_{5}\right|+\left|\boldsymbol{U}_{6}\right|+\left|\boldsymbol{U}_{8}\right|$ that enhances

623 the distribution of signals. The heme and globin are located in low intensities of

624 these oscillating components (Fig. 7b-e). These global oscillations occur only a

625 few times before dissipation (Gruia et al., 2008; Rosca et al., 2002). However,

626 there are not enough time points to determine the damping time constant, which

627 is roughly estimated to be 1-4 ps. Contrary to the common belief that low

628 frequency oscillations are due to the properties of the heme, here I attribute the

629 low frequency oscillation to the global response of protein and solvent as a whole

630 to a sudden molecular event - photodissociation of the ligand, where the

631 vibrational energy released from the photodissociation is quickly dissipated into

632 the environment.

\section{Discussion}

635 Are atomic orbitals observed?

636 It requires ultrahigh spatial resolution in a Fourier synthesis to accurately depict

637 the probability distribution of a single electron occupying its orbital. For

638 example, iron $3 d$ and sulfur $3 p$ densities in an $\mathrm{Fe}_{4} \mathrm{~S}_{4}$ cluster are described with

639 crystallographic data at a resolution of $0.48 \AA$ with an analytical technique called

640 charge-density analysis (Hirano et al., 2016), in which atoms are considered as

641 aspherical and modeled by spherical harmonic functions in contrast to spherical 
642 Gaussian functions used in everyday protein crystallography (Coppens, 1998).

643 Since the non-bonding inner electrons remain largely constant in a spherical

644 distribution as a whole, the inner electrons in a majority can easily overwhelm

645 the presence of the bonding electrons in a minority, let alone subtle changes in

646 electron transfer. Therefore, it is very difficult, if not impossible, to detect small

647 orbital changes upon bond rupture using regular electron density maps such as a

648 2Fo-Fc map. Difference Fourier technique is required as it is far more sensitive to

649 small changes (Henderson and Moffat, 1971), in which the constant, spherical

650 distribution of the inner electrons is self-canceled and the remaining densities

651 would reveal electron transfer. However, the reality is usually far less ideal

652 because of conformational changes. Atomic displacements involved in a

653 conformational change carry all electrons associated with the moving atoms and

654 result in significant positive and negative densities in a difference Fourier map,

655 that is, a spatial distribution of any gain and loss of electrons. Atomic

656 displacements such as a side chain rotation or the heme iron movement by a few

657 tenths of an $\AA$ again conceal the subtle signal of an electron transfer from one

658 orbital to another.

The difference maps derived here as SVD components represent partial,

661 variable signals in addition to the constant electron density map regardless of the

662 bonding change. An ultrahigh spatial resolution is required to depict slightly

663 aspherical features when an overwhelming spherical background is present.

664 Here, a combined use of difference Fourier technique and decomposition eases

665 the demand for spatial resolution to reveal subtle changes. Furthermore, the

666 notion that an ultrahigh spatial resolution is required for observations of orbital

667 changes contradicts the fact that there is no discontinuity in the probability

668 distribution of any electronic orbital. A reasonably high spatial resolution shall

669 be sufficient to analytically describe the smooth function of a gain or loss of an

670 electron. Therefore, the small amplitude of desired changes over a much greater

671 constant background hinders a reliable observation more than the lack of high

672 spatial frequencies does. This work presents the effectiveness of the strategy that

673 enables numerical deconvolution to resolve concurrent events so that the image

674 of a partial change, such as a single electron transfer, can be isolated from other

675 changes. At an available spatial resolution, the partial change due to a specific 
676 electronic event deconvoluted from much greater conformational changes could

677 be the remanece of orbital densities instead of an accurate image of these orbitals.

A limited experimental spatial resolution also raises a doubt whether some unusual features are the artifacts due to a Fourier truncation error. A Fourier truncation error may occur to several static datasets included in this analysis, in which the spatial resolutions are truncated to $1.75 \AA$ to match that of XFEL datasets. Such errors may appear as minor components after SVD (Figs. 2e and $4 \mathrm{f})$. When the average intensity of an experimental dataset naturally decays as function of the spatial resolution, Fourier truncation errors are damped to a minimum. Difference Fourier technique again self-cancels the remaining errors because both datasets involved in the subtraction would carry similar Fourier truncation errors if any. In addition, the applied weighting scheme (Ren et al., 2001, 2013; Šrajer et al., 2001; Ursby and Bourgeois, 1997) severely reduces any large differences in Fourier synthesis (Methods), which also prevents Fourier truncation errors. After the decomposition, proper rotations in the SVD space validate that the signals under discussion, such as the positive cubic network, is not due to Fourier truncation errors because of its correlation with the metadata. An artifact due to Fourier truncation errors, if so conclude, needs to be explained why it is correlated with a physical or chemical parameter.

The theoretical radii of the $3 d$ orbitals for an isolated Fe(II) cation are about

699 densities spread out many folds beyond the maxima (Waber and Cromer, 1965).

700 The majority of the iron $3 d$ orbital densities in an $\mathrm{Fe}_{4} \mathrm{~S}_{4}$ cluster span about $1 \AA$

701 from the nucleus as measured in a protein (Hirano et al., 2016). The observed

702 distances between the iron and the corners of the observed cubic densities are

703 significantly larger. Therefore, the observed cubic densities here are not an

704 accurate image of a probability distribution of the $3 d$ electrons. However, this

705 density distribution, given the available spatial resolution, is clearly influenced

706 by the unpaired $3 d$ electrons. The net gain of electron densities could be further

707 away from the nucleus compared to the maximum probability of these orbital.

708 For a practical purpose in protein crystallography, an accurate depiction of a $3 d$ 
Ren: Decomposition of ultrafast changes

709 orbital is not the task. Rather, direct observations of a specific electronic event

710 would provide unprecedented insight into the essence of biochemistry.

\section{Temporal resolution vs. resolution of heterogeneity}

713 Ultrashort XFEL pulses improve the time resolution to sub-ps or even tens of fs

714 compared to 100 ps at synchrotrons. This ultrafast time resolution, when

715 combined with the spatial resolution of crystallography, offers an unprecedented

716 opportunity for direct observation of transient structural changes in a

717 photochemical reaction as detail as individual electrons. However, due to their

718 highly dynamic and heterogeneous nature, it is difficult to experimentally isolate

719 short-lived structural species along the temporal dimension regardless of the

720 ultrafast time resolution. In other words, resolving the population difference

721 between two chemical species at one fs from the next does not mean that one can

722 resolve two distinct structures. As demonstrated by this work, analytical

723 methods such as SVD are needed to deconvolute them from mixtures of

724 intermediate species. The strategy we proposed for resolution of structural

725 heterogeneity (Ren et al., 2013) is highly effective for separating independent

726 electronic and atomic events that often coexist during a chemical reaction or a

727 biological process.

This work shows that it is possible to observe structural changes as small as a

731 signals can be correlated with their corresponding metadata by proper rotations,

732 that is, deconvolution after decomposition, thus allowing the origin of these

733 signals be revealed. I posit that all observations so far are the consequence,

734 rather than the cause, of the ligand dissociation. The proposed transient event of

735 metal-to-ligand charge transfer is not captured here, presumably because the

736 transferred electron is delocalized all over the porphyrin (Franzen et al., 2001).

737 Therefore, the fundamental questions remain for photolysis of $\mathrm{MbCO}$ : Upon the

738 absorption of a quantum of energy from a green photon, what initial electronic

739 events lead to a partitioning of the bonding electrons in two separate atoms, that

740 is, the bond rupture? What electronic events cause spin crossover? To image

741 these events directly, even higher time resolution is required to capture electron

742 density changes prior to and during bond rupture. 
Ren: Decomposition of ultrafast changes

\section{Future outlook of ultrafast dynamic crystallography}

745 Despite the recent developments in SFX and its applications to time-resolved

746 studies of a few model systems, it is yet premature to declare that the current

747 SFX platform applies to other systems of more significant biological importance

748 such as visual and microbial rhodopsins, plant and bacterial phytochromes,

749 photosynthetic reaction centers, and light-dependent DNA repair enzymes.

750 There are at least three major challenges. First, it is not practical to produce and

751 inject an astronomical number of nano or microcrystals of these proteins into an

752 XFEL beam to support data collections of a time series. The current serial

753 protocol used to obtain the $\mathrm{MbCO}$ and other data has a diminishingly small yield

754 of useful diffraction data, based on the ratio between the number of diffracting

755 crystals and the total number of crystals produced (Ren et al., 2018). That is to

756 say, the vast majority of the crystals have no chance to diffract X-ray even when

757 they are injected into the X-ray beam. Such a yield, defined differently from the

758 hit rate, is not sustainable for other photosensitive protein crystals. Several

759 "fixed target" implementations of crystal delivery systems have been reported,

760 including one that we have recently proposed (Ren et al., 2018). However, it

761 remains to be seen how effective they are in achieving a higher yield while

762 maintaining the diffraction quality.

Second, since the XFEL pulses usually carry insufficient bandwidths to fully integrate the Bragg reflections and in the meanwhile angular rotation of a crystal during an ultrashort XFEL pulse is not attainable, tens to hundreds of thousands of diffraction images are required to obtain integrated intensities for all Bragg reflections in a complete sampling of the reciprocal space. A possibility to solve this partiality problem is to use an X-ray beam with a significant convergence of about a degree (Ho et al., 2002; Spence et al., 2014), which will produce completely integrated intensities with a far fewer number of diffraction images, thereby alleviating the demand for a large quantity of purified proteins. 
also proposed a protocol to obtain difference signals that bypasses the integrated intensities of Bragg reflections. We reason that integrated intensities are not necessary if the dark and light diffraction images can be obtained within a single exposure of an XFEL pulse because the percentage change in integrated intensities of a given reflection, that is, the time-resolved signal, is already captured even if this reflection is partially measured in both dark and light conditions. A plan has been laid to implement this protocol, based on a dichromator instrument that achieves angular split and temporal delay at the same time (Ren and Yang, 2016). When each of these technical hurdles is overcome, direct observations of atomic and electronic movements during ultrafast processes will be closer to broad applications for many light sensitive crystals of great biological and medical significance.

\section{Code availability}

Computer software dynami ${ }^{\mathrm{TM}}$ is available from Renz Research, Inc.

\section{Acknowledgements}

The following database and software are used in this work: CCP4 (ccp4.ac.uk), Coot (www2.mrc-lmb.cam.ac.uk/Personal/pemsley/coot), dynamiX ${ }^{\mathrm{TM}}$ (Renz Research, Inc.), gnuplot (gnuplot.info), Mathematica (wolfram.com), PDB (rcsb.org), PHENIX (phenix-online.org), PyMOL (pymol.org), Python (python.org), and SciPy (scipy.org).

\section{Competing interests}

$\mathrm{ZR}$ is the founder of Renz Research, Inc. that currently holds the copyright of the computer software dynami $X^{\mathrm{TM}}$.

\section{References}

Abe, M., Kitagawa, T., and Kyogoku, Y. (1978). Resonance Raman spectra of octaethylporphyrinato-Ni(II) and meso-deuterated and $15 \mathrm{~N}$ substituted derivatives. II. A normal coordinate analysis. J. Chem. Phys. 69, 4526-4534.

Aranda, R., Levin, E.J., Schotte, F., Anfinrud, P.A., and Phillips, G.N. (2006). Time-dependent atomic coordinates for the dissociation of carbon monoxide from myoglobin. Acta Crystallogr. D Biol. Crystallogr. 62, 776-783. 4990-4994. 


\section{Ren: Decomposition of ultrafast changes}

814 Baldwin, J., and Chothia, C. (1979). Haemoglobin: the structural changes related to ligand

815 binding and its allosteric mechanism. J. Mol. Biol. 129, 175-200.

816 Bandara, S., Ren, Z., Lu, L., Xiaoli, Z., Shin, H., Zhao, K.-H., and Yang, X. (2017). Photoactivation 817 mechanism of a carotenoid-based photoreceptor. Proc. Natl. Acad. Sci. U. S. A. 114, 6286-6291.

818 Barends, T.R.M., Foucar, L., Ardevol, A., Nass, K., Aquila, A., Botha, S., Doak, R.B., Falahati, K., 819 Hartmann, E., Hilpert, M., et al. (2015). Direct observation of ultrafast collective motions in CO 820 myoglobin upon ligand dissociation. Science 350, 445-450.

821 Bionta, M.R., Lemke, H.T., Cryan, J.P., Glownia, J.M., Bostedt, C., Cammarata, M., Castagna, J.-C., 822 Ding, Y., Fritz, D.M., Fry, A.R., et al. (2011). Spectral encoding of x-ray / optical relative delay.

823 Opt. Express 19, 21855.

824 Bionta, M.R., Hartmann, N., Weaver, M., French, D., Nicholson, D.J., Cryan, J.P., Glownia, J.M., 825 Baker, K., Bostedt, C., Chollet, M., et al. (2014). Spectral encoding method for measuring the 826 relative arrival time between x-ray/optical pulses. Rev. Sci. Instrum. 85, 083116.

827 Bourgeois, D., Vallone, B., Schotte, F., Arcovito, A., Miele, A.E., Sciara, G., Wulff, M., Anfinrud, 828 P., and Brunori, M. (2003). Complex landscape of protein structural dynamics unveiled by 829 nanosecond Laue crystallography. Proc. Natl. Acad. Sci. 100, 8704-8709.

830 Bourgeois, D., Vallone, B., Arcovito, A., Sciara, G., Schotte, F., Anfinrud, P.A., and Brunori, M. 831 (2006). Extended subnanosecond structural dynamics of myoglobin revealed by Laue 832 crystallography. Proc. Natl. Acad. Sci. 103, 4924-4929.

833 Bourgeois, D., Schotte, F., Brunori, M., and Vallone, B. (2007). Time-resolved methods in 834 biophysics. 6. Time-resolved Laue crystallography as a tool to investigate photo-activated protein 835 dynamics. Photochem. Photobiol. Sci. 6, 1047.

836 Brinkmann, L.U.L., and Hub, J.S. (2016). Ultrafast anisotropic protein quake propagation after CO 837 photodissociation in myoglobin. Proc. Natl. Acad. Sci. 113, 10565-10570.

838 Chaika, A.N. (2014). Visualization of electron orbitals in scanning tunneling microscopy. JETP 839 Lett. 99, 731-741.

840 Champion, P.M. (2005). Chemistry: Following the flow of energy in biomolecules. Science 310, $841980-982$.

842 Chu, K., Vojtchovský, J., McMahon, B.H., Sweet, R.M., Berendzen, J., and Schlichting, I. (2000). 843 Structure of a ligand-binding intermediate in wild-type carbonmonoxy myoglobin. Nature 403, 844 921-923.

845 Clifton, I.J., Duke, E.M.H., Wakatsuki, S., and Ren, Z. (1997). Evaluation of Laue diffraction 846 patterns. Meth Enzym. 277, 448-467.

847 Coppens, P. (1998). Charge-Density Analysis at the Turn of the Century. Acta Crystallogr. A 54, $848 \quad 779-788$.

849 Coquelle, N., Sliwa, M., Woodhouse, J., Schirò, G., Adam, V., Aquila, A., Barends, T.R.M., Boutet, 850 S., Byrdin, M., Carbajo, S., et al. (2017). Chromophore twisting in the excited state of a

851 photoswitchable fluorescent protein captured by time-resolved serial femtosecond

852 crystallography. Nat. Chem. 10, 31-37.

853 Dunietz, B.D., Dreuw, A., and Head-Gordon, M. (2003). Initial steps of the photodissociation of 854 the CO ligated heme group. J. Phys. Chem. B 107, 5623-5629. 
Ren: Decomposition of ultrafast changes

855 Falahati, K., Tamura, H., Burghardt, I., and Huix-Rotllant, M. (2017). Quantum dynamics of 856 ultrafast heme-carbon monoxide photolysis and spin-crossover. ArXiv170903402 Cond-Mat 857 Physicsphysics.

858 Falahati, K., Tamura, H., Burghardt, I., and Huix-Rotllant, M. (2018). Ultrafast carbon monoxide 859 photolysis and heme spin-crossover in myoglobin via nonadiabatic quantum dynamics. Nat.

860 Commun. 9, 4502.

861 Franzen, S., Bohn, B., Poyart, C., DePillis, G., Boxer, S.G., and Martin, J.-L. (1995). Functional 862 aspects of ultra-rapid heme doming in hemoglobin, myoglobin, and the myoglobin mutant 863 H93G. J. Biol. Chem. 270, 1718-1720.

864 Franzen, S., Kiger, L., Poyart, C., and Martin, J.-L. (2001). Heme photolysis occurs by ultrafast 865 excited state metal-to-ring charge transfer. Biophys. J. 80, 2372-2385.

866 Fujisaki, H., and Straub, J.E. (2005). Vibrational energy relaxation in proteins. Proc. Natl. Acad.

867 Sci. 102, 6726-6731.

868 Gruia, F., Kubo, M., Ye, X., and Champion, P.M. (2008). Investigations of vibrational coherence in 869 the low-frequency region of ferric heme proteins. Biophys. J. 94, 2252-2268.

870 Haldrup, K., Harlang, T., Christensen, M., Dohn, A., van Driel, T.B., Kjær, K.S., Harrit, N.,

871 Vibenholt, J., Guerin, L., Wulff, M., et al. (2011). Bond shortening (1.4 A) in the singlet and triplet 872 excited states of [Ir2(dimen)4]2+ in solution determined by time-resolved X-ray scattering. Inorg 873 Chem 50, 9329-9336.

874 Henderson, R., and Moffat, J.K. (1971). The difference Fourier technique in protein 875 crystallography: errors and their treatment. Acta Cryst B27, 1414-1420.

876 Henry, E.R., and Hofrichter, J. (1992). Singular value decomposition: Application to analysis of 877 experimental data. In Numerical Computer Methods, (Academic Press), pp. 129-192.

878 Henry, E.R., Levitt, M., and Eaton, W.A. (1985). Molecular dynamics simulation of 879 photodissociation of carbon monoxide from hemoglobin. Proc. Natl. Acad. Sci. U. S. A. 82, 20348802038.

881 Hirano, Y., Takeda, K., and Miki, K. (2016). Charge-density analysis of an iron-sulfur protein at 882 an ultra-high resolution of $0.48 \AA$ A. Nature $534,281-284$.

883 Ho, J.X., Ruble, J.R., McInnis, T.R., Carter, D.C., Huang, H., and Gibson, W.M. (2002).

884 Convergent-beam method in macromolecular crystallography. Acta Crystallogr. D Biol.

885 Crystallogr. 58, 2087-2095.

886 Hodel, A., Kim, S.H., and Brünger, A.T. (1992). Model bias in macromolecular crystal structures. 887 Acta Crystallogr. A 48, 851-858.

888 Humphreys, C.J. (1999). Electrons seen in orbit. Nature 401, 21-22.

889 Itatani, J., Levesque, J., Zeidler, D., Niikura, H., Pépin, H., Kieffer, J.C., Corkum, P.B., and 890 Villeneuve, D.M. (2004). Tomographic imaging of molecular orbitals. Nature 432, 867-871.

891 Jung, Y.O., Lee, J.H., Kim, J., Schmidt, M., Moffat, K., Šrajer, V., and Ihee, H. (2013). Volume892 conserving trans-cis isomerization pathways in photoactive yellow protein visualized by 893 picosecond X-ray crystallography. Nat. Chem. 5, 212-220.

894 Kachalova, G.S., Popov, A.N., and Bartunik, H.D. (1999). A steric mechanism for inhibition of CO 895 binding to heme proteins. Science $284,473-476$. 
Ren: Decomposition of ultrafast changes

896 Karunakaran, V., Benabbas, A., Sun, Y., Zhang, Z., Singh, S., Banerjee, R., and Champion, P.M.

897 (2010). Investigations of low-frequency vibrational dynamics and ligand binding kinetics of

898 cystathionine $\beta$-synthase. J. Phys. Chem. B 114, 3294-3306.

899 Karunakaran, V., Sun, Y., Benabbas, A., and Champion, P.M. (2014). Investigations of the low

900 frequency modes of ferric cytochrome $\mathrm{c}$ using vibrational coherence spectroscopy. J. Phys. Chem.

901 B 118, 6062-6070.

902 Kim, T.H., Mehrabi, P., Ren, Z., Sljoka, A., Ing, C., Bezginov, A., Ye, L., Pomès, R., Prosser, R.S., 903 and Pai, E.F. (2017). The role of dimer asymmetry and protomer dynamics in enzyme catalysis.

904 Science 355, eaag2355.

905 Kitagawa, T., Haruta, N., and Mizutani, Y. (2002). Time-resolved resonance Raman study on 906 ultrafast structural relaxation and vibrational cooling of photodissociated carbonmonoxy 907 myoglobin. Biopolymers 67, 207-213.

908 Kuczera, K., Lambry, J.-C., Martin, J.-L., and Karplus, M. (1993). Nonexponential relaxation after 909 ligand dissociation from myoglobin: A molecular dynamics simulation. Proc. Natl. Acad. Sci. U.

910 S. A. $90,5805-5807$.

911 Levantino, M., Yorke, B.A., Monteiro, D.C., Cammarata, M., and Pearson, A.R. (2015a). Using

912 synchrotrons and XFELs for time-resolved X-ray crystallography and solution scattering

913 experiments on biomolecules. Curr. Opin. Struct. Biol. 35, 41-48.

914 Levantino, M., Schirò, G., Lemke, H.T., Cottone, G., Glownia, J.M., Zhu, D., Chollet, M., Ihee, H.,

915 Cupane, A., and Cammarata, M. (2015b). Ultrafast myoglobin structural dynamics observed with

916 an X-ray free-electron laser. Nat. Commun. 6, 6772.

917 Mikhailovskij, I.M., Sadanov, E.V., Mazilova, T.I., Ksenofontov, V.A., and Velicodnaja, O.A.

918 (2009). Imaging the atomic orbitals of carbon atomic chains with field-emission electron

919 microscopy. Phys. Rev. B 80.

920 Mizutani, Y. (1997). Direct observation of cooling of heme upon photodissociation of

921 carbonmonoxy myoglobin. Science $278,443-446$.

922 Mulder, P. (2011). Are orbitals observable? Int. J. Philos. Chem. 17, 24-35.

923 Nagy, A.M., Raicu, V., and Miller, R.J.D. (2005). Nonlinear optical studies of heme protein 924 dynamics: Implications for proteins as hybrid states of matter. Biochim. Biophys. Acta BBA 925 Proteins Proteomics 1749, 148-172.

926 Nango, E., Royant, A., Kubo, M., Nakane, T., Wickstrand, C., Kimura, T., Tanaka, T., Tono, K.,

927 Song, C., Tanaka, R., et al. (2016). A three-dimensional movie of structural changes in

928 bacteriorhodopsin. Science 354, 1552-1557.

929 Nogly, P., Weinert, T., James, D., Carbajo, S., Ozerov, D., Furrer, A., Gashi, D., Borin, V.,

930 Skopintsev, P., Jaeger, K., et al. (2018). Retinal isomerization in bacteriorhodopsin captured by a 931 femtosecond $\mathrm{x}$-ray laser. Science eaat0094.

932 Ogilvie, J.F. (2011). Is a molecular orbital measurable by means of tomographic imaging? Found.

933 Chem. 13, 87-91.

934 Pande, K., Hutchison, C.D.M., Groenhof, G., Aquila, A., Robinson, J.S., Tenboer, J., Basu, S., 935 Boutet, S., DePonte, D.P., Liang, M., et al. (2016). Femtosecond structural dynamics drives the 936 trans/cis isomerization in photoactive yellow protein. Science 352, 725-729. 
Ren: Decomposition of ultrafast changes

937 Patera, L.L., Queck, F., Scheuerer, P., and Repp, J. (2019). Mapping orbital changes upon electron 938 transfer with tunnelling microscopy on insulators. Nature 566, 245-248.

939 Puschnig, P., Berkebile, S., Fleming, A.J., Koller, G., Emtsev, K., Seyller, T., Riley, J.D., Ambrosch940 Draxl, C., Netzer, F.P., and Ramsey, M.G. (2009). Reconstruction of molecular orbital densities 941 from photoemission data. Science 326, 702-706.

942 Ren, Z. (2013a). Reaction trajectory revealed by a joint analysis of Protein Data Bank. PLoS ONE $9438, \mathrm{e} 77141$.

944 Ren, Z. (2013b). Reverse engineering the cooperative machinery of human hemoglobin. PLoS 945 ONE 8, e77363.

946 Ren, Z. (2016). Molecular events during translocation and proofreading extracted from 200 static 947 structures of DNA polymerase. Nucleic Acids Res. 6, 1-13.

948 Ren, Z., and Yang, X. (2016). Angular-split/temporal-delay approach to ultrafast protein 949 dynamics at XFELs. Acta Crystallogr. Sect. Struct. Biol. 72, 871-882.

950 Ren, Z., Bourgeois, D., Helliwell, J.R., Moffat, K., Srajer, V., and Stoddard, B.L. (1999). Laue 951 crystallography: coming of age. J Synchrotron Rad 6, 891-917.

952 Ren, Z., Perman, B., Srajer, V., Teng, T.-Y., Pradervand, C., Bourgeois, D., Schotte, F., Ursby, T., 953 Kort, R., Wulff, M., et al. (2001). A molecular movie at 1.8 Å resolution displays the photocycle of 954 photoactive yellow protein, a eubacterial blue-light receptor, from nanoseconds to seconds. 955 Biochemistry 40, 13788-13801.

956 Ren, Z., Šrajer, V., Knapp, J.E., and Royer, W.E. (2012). Cooperative macromolecular device 957 revealed by meta-analysis of static and time-resolved structures. Proc. Natl. Acad. Sci. U. S. A. $958 \quad 109,107-112$.

959 Ren, Z., Chan, P.W.Y., Moffat, K., Pai, E.F., Royer, W.E., Šrajer, V., and Yang, X. (2013). Resolution 960 of structural heterogeneity in dynamic crystallography. Acta Cryst D69, 946-959.

961 Ren, Z., Ayhan, M., Bandara, S., Bowatte, K., Kumarapperuma, I., Gunawardana, S., Shin, H., 962 Wang, C., Zeng, X., and Yang, X. (2018). Crystal-on-crystal chips for in situ serial diffraction at 963 room temperature. Lab. Chip 2246-2256.

964 Repp, J., Meyer, G., Stojković, S.M., Gourdon, A., and Joachim, C. (2005). Molecules on insulating 965 films: Scanning-tunneling microscopy imaging of individual molecular orbitals. Phys. Rev. Lett. 96694.

967 Rosca, F., Kumar, A.T.N., Ionascu, D., Ye, X., Demidov, A.A., Sjodin, T., Wharton, D., Barrick, D., 968 Sligar, S.G., Yonetani, T., et al. (2002). Investigations of anharmonic low-frequency oscillations in 969 heme proteins. J. Phys. Chem. A 106, 3540-3552.

970 Scerri, E.R. (2000). Have orbitals really been observed? J. Chem. Educ. 77, 1492.

971 Schmidt, M., Rajagopal, S., Ren, Z., and Moffat, K. (2003). Application of singular value 972 decomposition to the analysis of time-resolved macromolecular X-ray data. Biophys. J. 84, 21129732129.

974 Schotte, F., Lim, M., Jackson, T.A., Smirnov, A.V., Soman, J., Olson, J.S., Phillips, G.N., Wulff, M., 975 and Anfinrud, P.A. (2003). Watching a protein as it functions with 150-ps time-resolved X-ray 976 crystallography. Science 300, 1944-1947. 
Ren: Decomposition of ultrafast changes

977 Schotte, F., Cho, H.S., Kaila, V.R.I., Kamikubo, H., Dashdorj, N., Henry, E.R., Graber, T.J.,

978 Henning, R., Wulff, M., Hummer, G., et al. (2012). Watching a signaling protein function in real

979 time via 100-ps time-resolved Laue crystallography. Proc. Natl. Acad. Sci. 109, 19256-19261.

980 Schwarz, W.H.E. (2006). Measuring Orbitals: Provocation or Reality? Angew. Chem. Int. Ed. 45, $981 \quad 1508-1517$.

982 Spence, J.C.H., Zatsepin, N.A., and Li, C. (2014). Coherent convergent-beam time-resolved X-ray 983 diffraction. Philos. Trans. R. Soc. B Biol. Sci. 369, $20130325-20130325$.

984 Šrajer, V., Teng, T. -y., Ursby, T., Pradervand, C., Ren, Z., Adachi, S. -i., Schildkamp, W.,

985 Bourgeois, D., Wulff, M., and Moffat, K. (1996). Photolysis of the carbon monoxide complex of

986 myoglobin: nanosecond time-resolved crystallography. Science 274, 1726-1729.

987 Šrajer, V., Ren, Z., Teng, T.-Y., Schmidt, M., Ursby, T., Bourgeois, D., Pradervand, C., Schildkamp, 988 W., Wulff, M., and Moffat, K. (2001). Protein conformational relaxation and ligand migration in 989 myoglobin: A nanosecond to millisecond molecular movie from time-resolved Laue X-ray 990 diffraction. Biochemistry 40, 13802-13815.

991 Stapelfeldt, H. (2004). Electrons frozen in motion. Nature 432, 809-810.

992 Stodolna, A.S., Rouzée, A., Lépine, F., Cohen, S., Robicheaux, F., Gijsbertsen, A., Jungmann, J.H., 993 Bordas, C., and Vrakking, M.J.J. (2013). Hydrogen atoms under magnification: Direct observation 994 of the nodal structure of Stark states. Phys. Rev. Lett. 110.

995 Tenboer, J., Basu, S., Zatsepin, N., Pande, K., Milathianaki, D., Frank, M., Hunter, M., Boutet, S., 996 Williams, G.J., Koglin, J.E., et al. (2014). Time-resolved serial crystallography captures high997 resolution intermediates of photoactive yellow protein. Science 346, 1242-1246.

998 Ursby, T., and Bourgeois, D. (1997). Improved estimation of structure-factor difference

999 amplitudes from poorly accurate data. Acta Crystallogr. A 53, 564-575.

1000 Vozzi, C., Negro, M., Calegari, F., Sansone, G., Nisoli, M., De Silvestri, S., and Stagira, S. (2011). 1001 Generalized molecular orbital tomography. Nat. Phys. 7, 822-826.

1002 Waber, J.T., and Cromer, D.T. (1965). Orbital radii of atoms and ions. J. Chem. Phys. 42, 411610034123.

1004 Yang, X., Ren, Z., Kuk, J., and Moffat, K. (2011). Temperature-scan cryocrystallography reveals 1005 reaction intermediates in bacteriophytochrome. Nature 479, 428-432.

1006 Zeng, X., Ren, Z., Wu, Q., Fan, J., Peng, P.-P., Tang, K., Zhang, R., Zhao, K.-H., and Yang, X. 1007 (2015). Dynamic crystallography reveals early signaling events in ultraviolet photoreceptor 1008 UVR8. Nat Plants 1, 14006.

1009 Zuo, J.M., Kim, M., O'Keeffe, M., and Spence, J.C.H. (1999). Direct observation of d-orbital holes 1010 and $\mathrm{Cu}-\mathrm{Cu}$ bonding in $\mathrm{Cu} 2 \mathrm{O}$. Nature 401, 49-52.

1011 
bioRxiv preprint doi: https://doi.org/10.1101/634071; this version posted July 12,2019 . The copyright holder for this preprint (which was not certified by peer review) is the author/funder. All rights reserved. No reuse allowed without permission.

Ren: Decomposition of ultrafast changes

\section{Figures and Legends}

1013

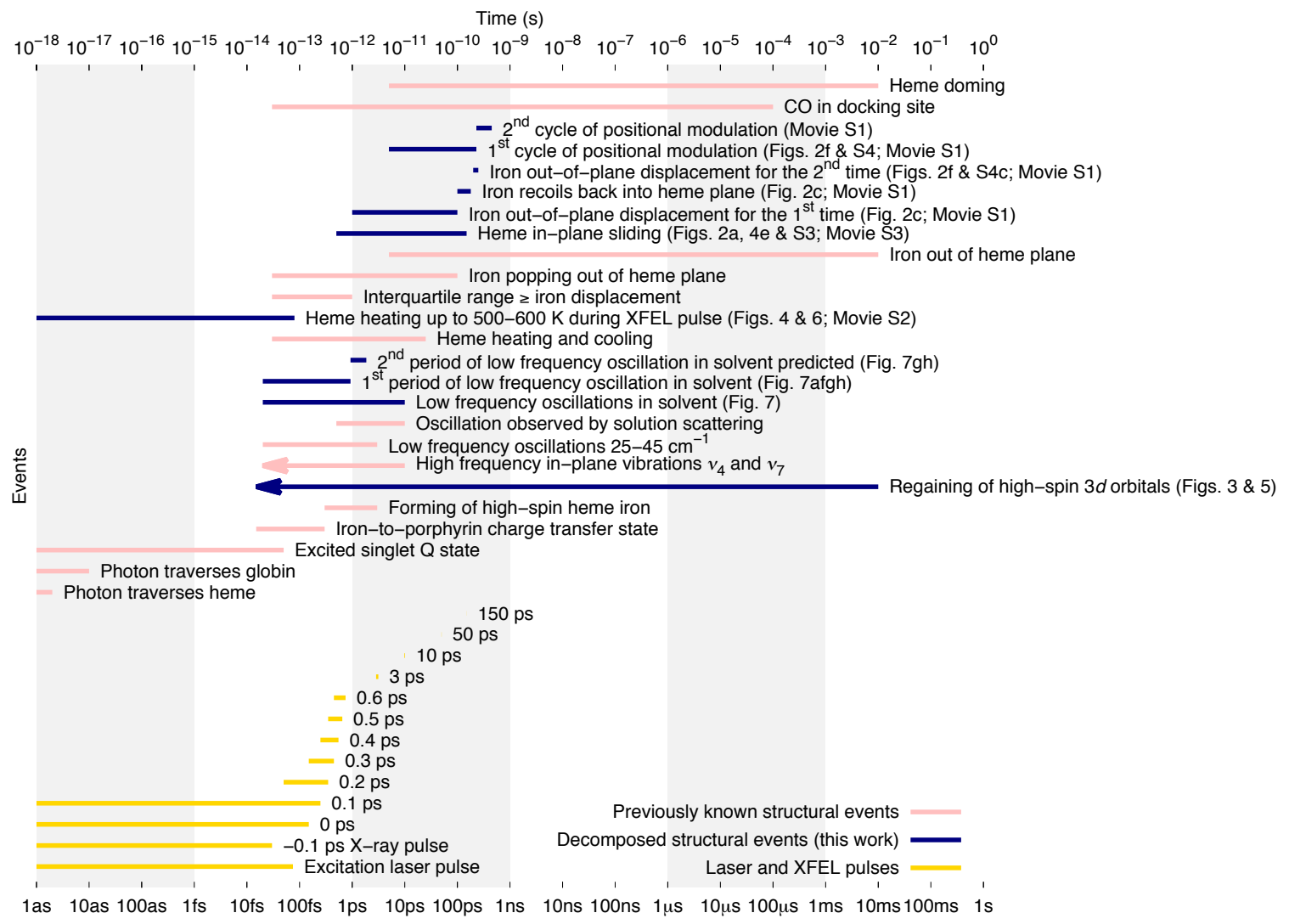

1016 Figure 1. A Gantt chart summarizing structural events during photodissociation 1017 of MbCO. Previously known structural events are in pink. The excitation laser 1018 pulse and XFEL pulses are in yellow. The newly observed structural events from 1019 decomposition of the serial crystallographic datasets are in dark blue. 
Ren: Decomposition of ultrafast changes
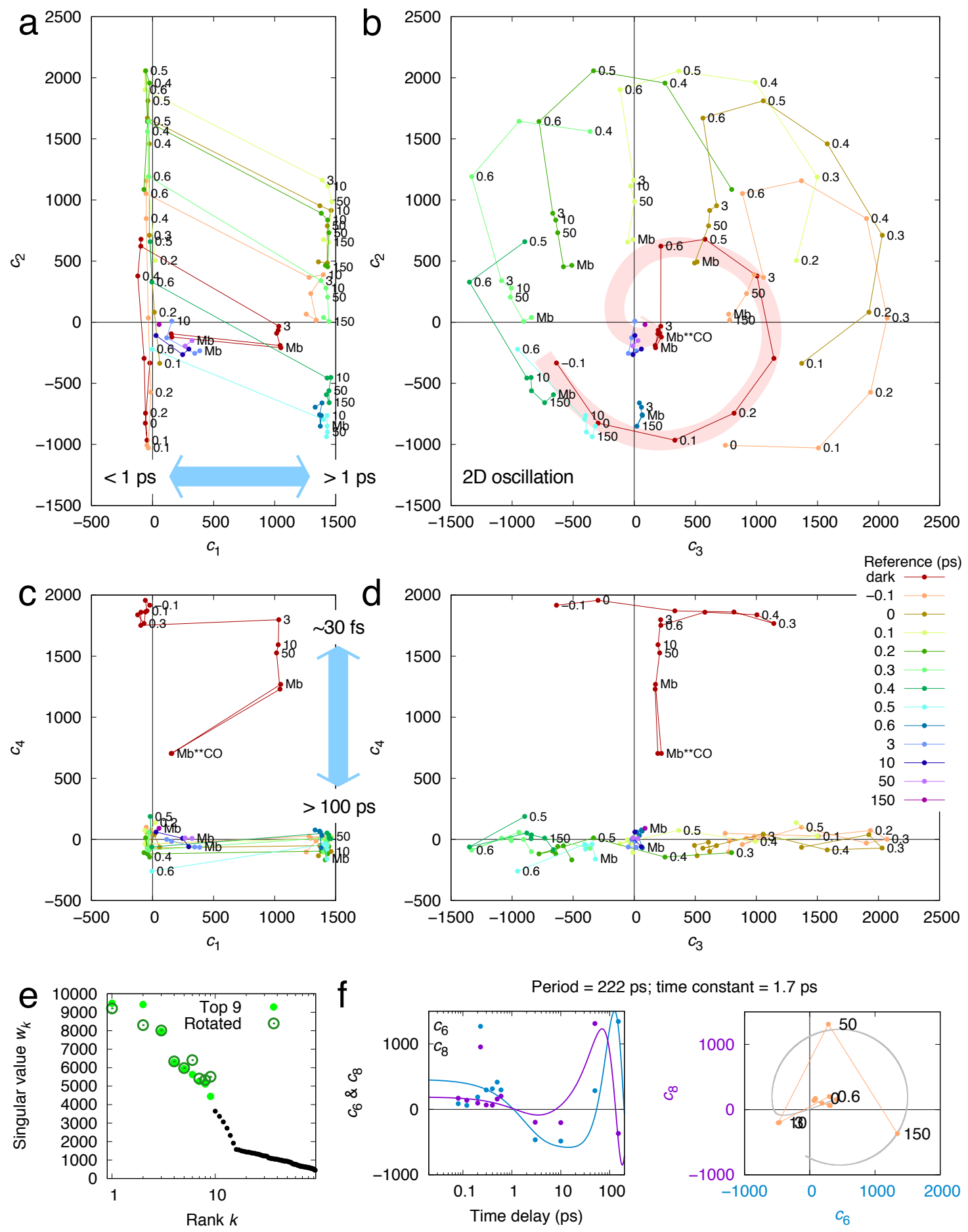

1021

1022

1023 Figure 2. SVD of 93 difference maps around the heme site. These difference

1024 maps are divided into several series. Each series consists of the difference maps

1025 produced from subtracting the same reference (Methods and Table S1). Each of 
1026 the 93 difference maps can be expressed as a linear combination of many

1027 components $c_{1} \boldsymbol{U}_{1}+c_{2} \boldsymbol{U}_{2}+\ldots$ after the decomposition. Among a common set of

1028 basis components $\boldsymbol{U}_{k}(k=1,2, \ldots)$ shared by all difference maps, also known as

1029 left singular vectors in linear algebra, the first few components are the most

1030 significant. Each of the difference maps requires a coefficient set $c_{k}$ for the linear

1031 combination. This coefficient set varies from one map to the other, that is, a

1032 function of the delay time or other metadata associate with the datasets. Each

1033 coefficient $c_{k}$ indicates how much of the corresponding component $\boldsymbol{U}_{k}$ exists in

1034 the linear combination. Each coefficient set is derived from a right singular

1035 vector as traditionally known in linear algebra (Methods). These coefficients

1036 produced by SVD should not be confused with the Fourier coefficients used to

1037 synthesize each map. (a-d) Orthographic projections of coefficients in some of

1038 the significant dimensions. Each difference map is represented by a dot at the

1039 location that marks the coefficients associated with the major components $\boldsymbol{U}_{1}$

1040 through $\boldsymbol{U}_{4}$. Difference maps in a series are connected by lines in a

1041 corresponding color. The multi-dimensional space is projected to various planes

1042 and tiled together so that any two adjacent panels from (a) to (d), horizontal or

1043 vertical, can be imagined erecting a three-dimensional subspace. (e) Singular

1044 values indicating the significance of the components are sorted in a descending

1045 order of their values before a rotation. A multi-dimensional rotation alters their

1046 values. (f) Coefficients $c_{6}$ and $c_{8}$ of the $-F_{-0.1}$ series in yellow are least-squares

1047 fitted with a two-dimensional oscillation (Methods). 


\section{$\boldsymbol{U}_{4}$ of difference maps at heme site}
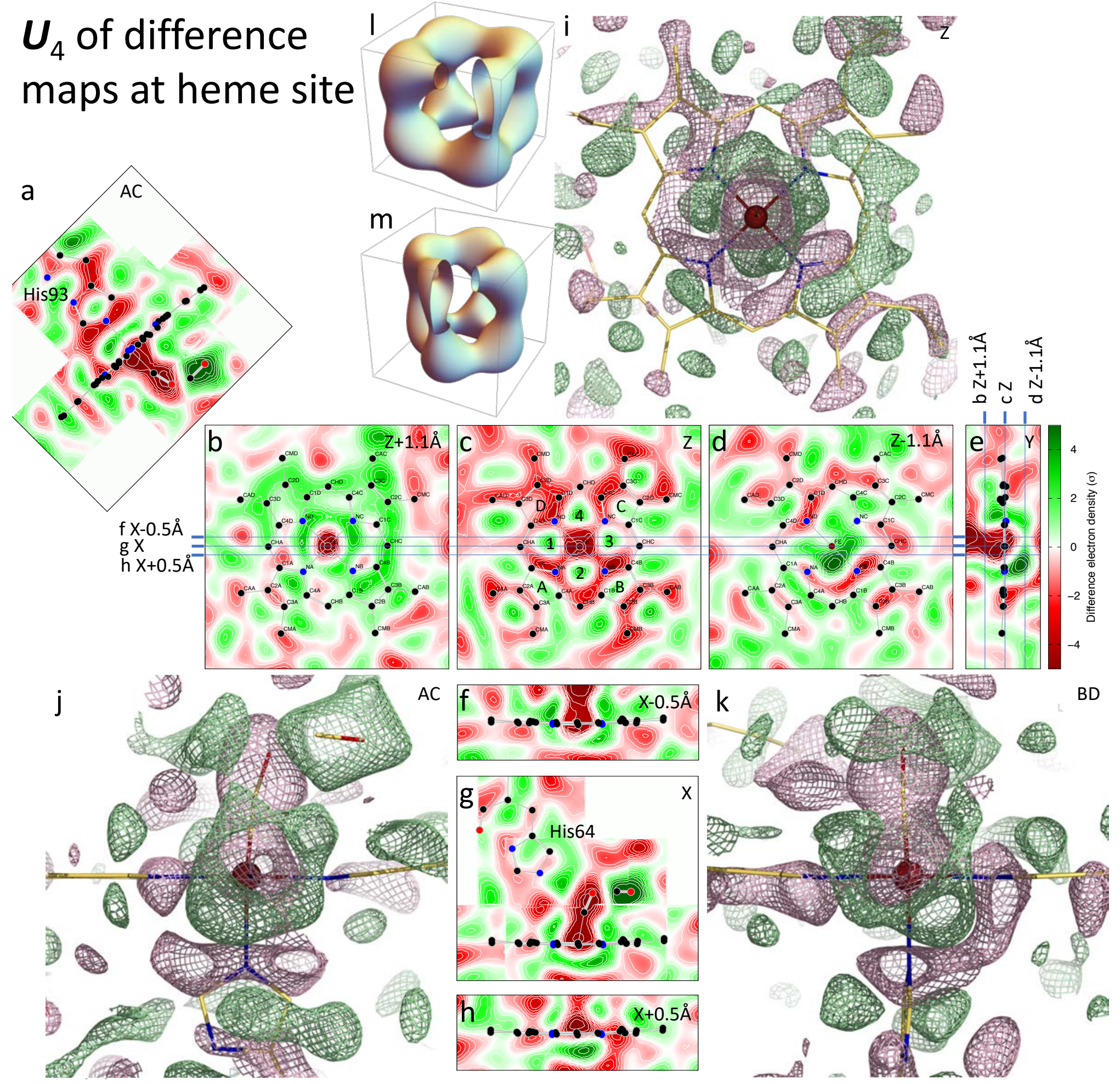

1050

1051 Figure 3. Ultrafast formation of high-spin $3 d$ orbitals of the heme iron. Each

1052 component resulting from SVD, that is, a left singular vector, can also be

1053 presented as a difference map. The fourth component $\boldsymbol{U}_{4}$ of the difference maps 
Ren: Decomposition of ultrafast changes

1054 captures a cubic shaped positive density network. It is rendered as a difference

1055 map in two styles, two-dimensional cross sections and three-dimensional

1056 contours. The corresponding coefficient $c_{4}$ is plotted in Fig. 2cd. (a-h) Images of

1057 two-dimensional cross sections through the component difference map $\boldsymbol{U}_{4}$. Cross

1058 sections defined in Fig. S1 are labeled in the top right corner of each panel. Non-

1059 centric cross sections are marked to indicate small offsets from the centric cross

1060 sections. The structure of the heme, CO ligand in both bound and docked

1061 positions, and some side chains are also projected onto the images. C, N, O, and

1062 Fe atoms are in black, blue, red, and rusty dots, respectively. The imidazole

1063 rings of the distal His64 and proximal His93 are approximately coplanar with $X$

1064 and AC cross sections, respectively. Two propionic acid side chains of AD rings

1065 extend to the left in (bcd). But they are not coplanar with these cross sections. (i-

1066 k) Three-dimensional mesh contours superimposed over a structure are

1067 traditionally rendered to display electron density maps. Green and red meshes

1068 are positive and negative contours at $\pm 7 \sigma$, respectively. ( 1 and $\mathrm{m}$ ) Theoretical

1069 contours of probability distributions of electrons in $3 d$ orbitals of iron. The

1070 combined probability distributions of $3 d_{x z}$ and $3 d_{y z}$ with $3 d_{x y}$ (l) or with $3 d_{x^{2}-y^{2}}$

1071 (m) are both in the shape of a cube with the largest probability at the eight

1072 corners. A 1/16 cutaway in each contour shows the cross sections. This

1073 characteristic shape of electron distribution is observed in $\boldsymbol{U}_{4}$ of the difference

1074 maps.

1075 
Ren: Decomposition of ultrafast changes
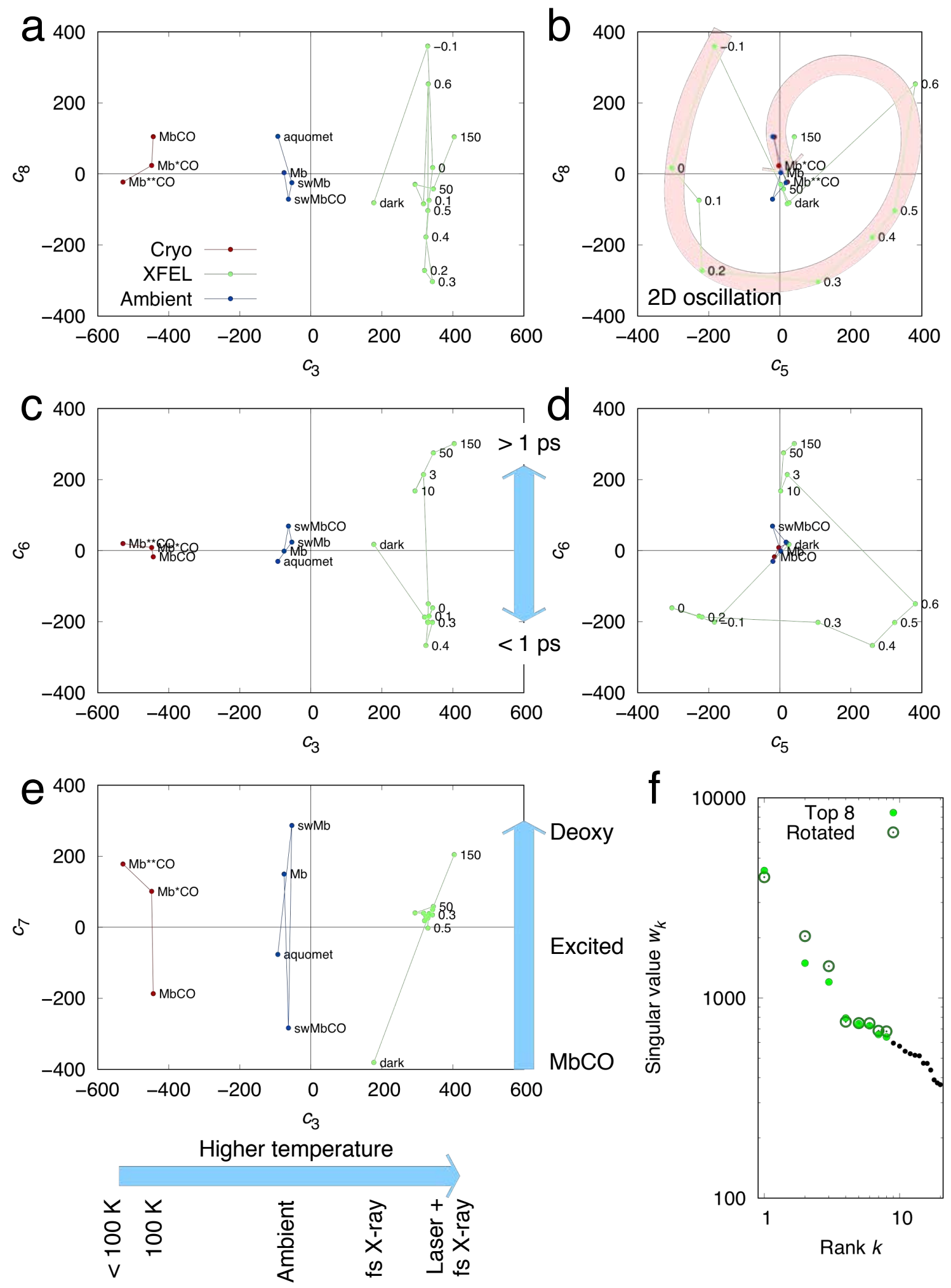

1077

1078 Figure 4. SVD of 20 simulated annealing omit maps (SAOMs) around the heme 1079 site. The first component is the average of all omit maps (Fig. S5). (a-e) Each 
Ren: Decomposition of ultrafast changes

1080 omit map is represented by a dot at the location that marks the coefficients

1081 associated with the major components. SAOMs derived from the structures at

1082 cryo and ambient temperatures are in red and blue, respectively. SAOMs from

1083 the XFEL datasets are in green. The multi-dimensional space is projected to

1084 various planes and tiled together so that any two adjacent panels from (a) to (e),

1085 horizontal or vertical, can be imagined erecting a three-dimensional subspace.

1086 (b) Coefficients $c_{5}$ and $c_{8}$ show a damped two-dimensional oscillation as marked

1087 by the curved arrow. (f) Singular values indicating the significance of the

1088 components are sorted in a descending order of their values before a multi-

1089 dimensional rotation.

1090 
Ren: Decomposition of ultrafast changes

\section{$\boldsymbol{U}_{7}$ of SAOMs at heme site}
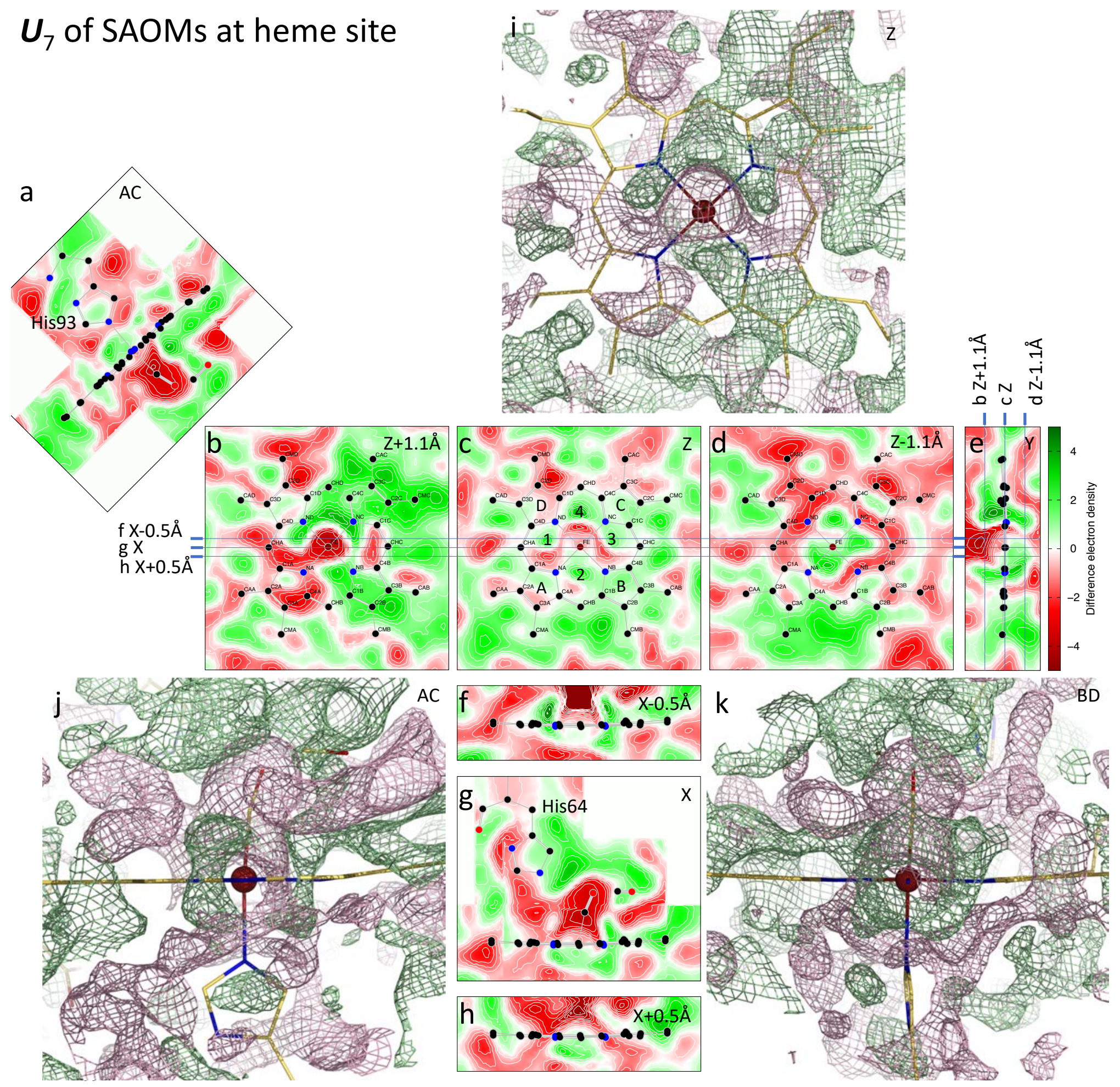

1092

1093 Figure 5. The seventh component of SAOMs correlated with photolysis at all

1094 temperatures. The coefficient corresponding to $\boldsymbol{U}_{7}$ of SAOMs increases as the

1095 reaction of ligand dissociation proceeds from $\mathrm{MbCO}$ to deoxy $\mathrm{Mb}$ in static, cryo- 
Ren: Decomposition of ultrafast changes

1096 trapping, and time-resolved XFEL experiments (Fig. 4e). The hypothetical

1097 transition from $\mathrm{MbCO}$ to deoxy $\mathrm{Mb}$ at $0 \mathrm{~K}$ is shown in Movie S3. (a-h) Cross

1098 sections through the seventh component $\boldsymbol{U}_{7}$. A cubic network of positive

1099 densities is marked 1-4 in (c). (i-k) Three-dimensional meshes of this component

1100 in green and red are contoured at $\pm 15 \sigma$, respectively. See Fig. 3 legend for more

1101 detail.

1102 
Ren: Decomposition of ultrafast changes

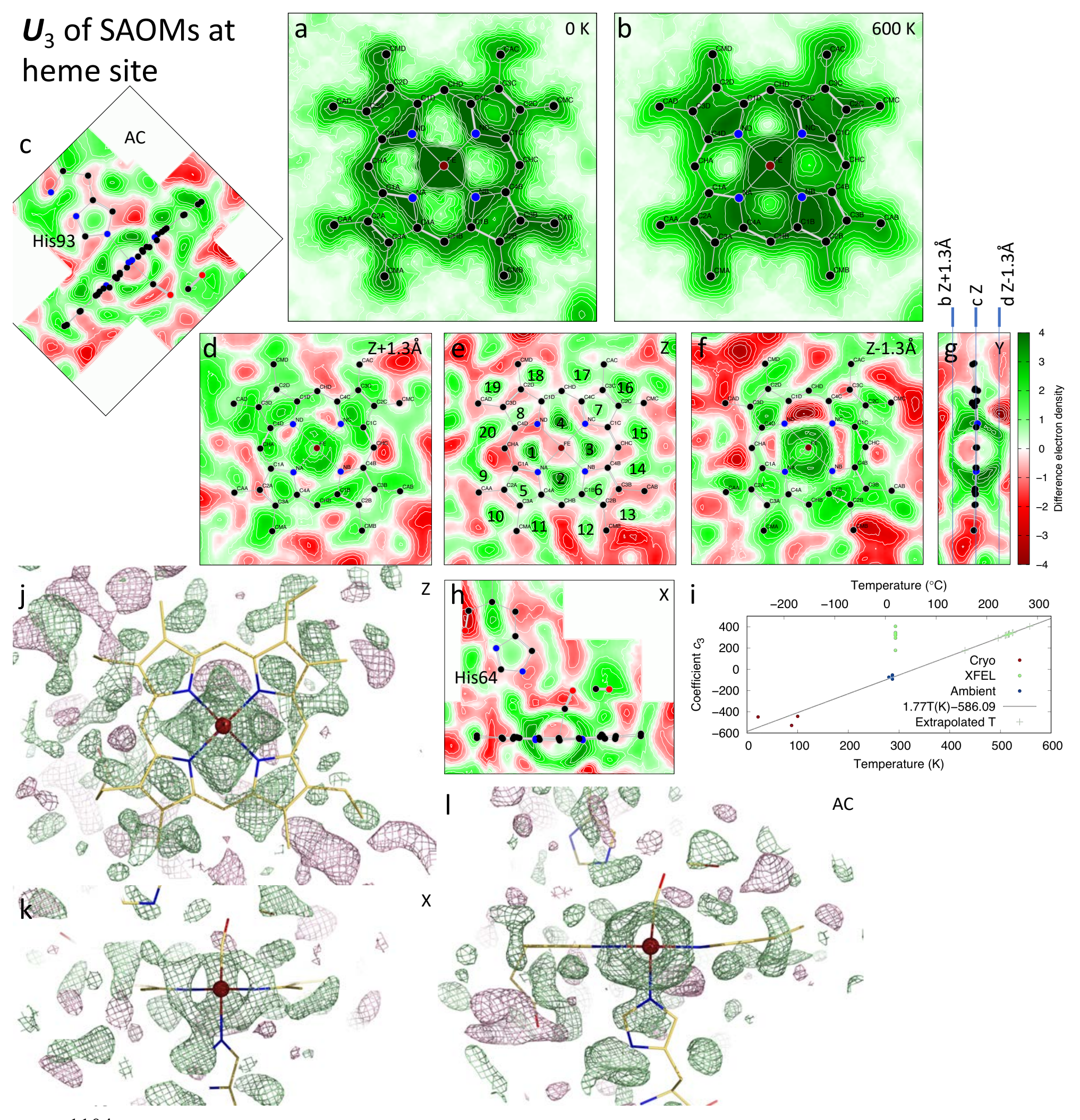

1104 
Ren: Decomposition of ultrafast changes

1105 Figure 6. Thermal vibrations isolated from the chemical signals of photolysis.

1106 The third component of the SAOMs $\boldsymbol{U}_{3}$ is highly correlated with the sample

1107 temperature (Fig. 4ace). A linear extrapolation based on the datasets available at

1108 cryo and ambient temperatures predicts that the instantaneous local temperature

1109 jump of the heme may have exceeded $500 \mathrm{~K}$ in the XFEL datasets (i). ( $a$ and $b$ )

1110 Linear combinations of $900 U_{1}-586 U_{3}$ and $900 U_{1}+476 U_{3}$ reconstitute the omit

1111 maps of the heme at 0 and $600 \mathrm{~K}$, respectively, excluding all chemical signals

1112 related to photolysis, where the coefficient $c_{1}=900$ of $\boldsymbol{U}_{1}$ is nearly constant for all

1113 omit maps. The transition from 0 to $600 \mathrm{~K}$ free of the chemical signals from

1114 photolysis is shown in Movie S2. (c-h) Cross sections through the third

1115 component $\boldsymbol{U}_{3}$. Positive densities in 20 pillars connecting two sheets of positive

1116 densities above and below the heme in the cross sections of $Z \pm 1.3 \AA$ are marked

1117 in (e). ( $\mathrm{j}-1)$ Three-dimensional meshes of this component in green and red are

1118 contoured at $\pm 15 \sigma$, respectively. See Fig. 3 legend for more detail. 
Ren: Decomposition of ultrafast changes

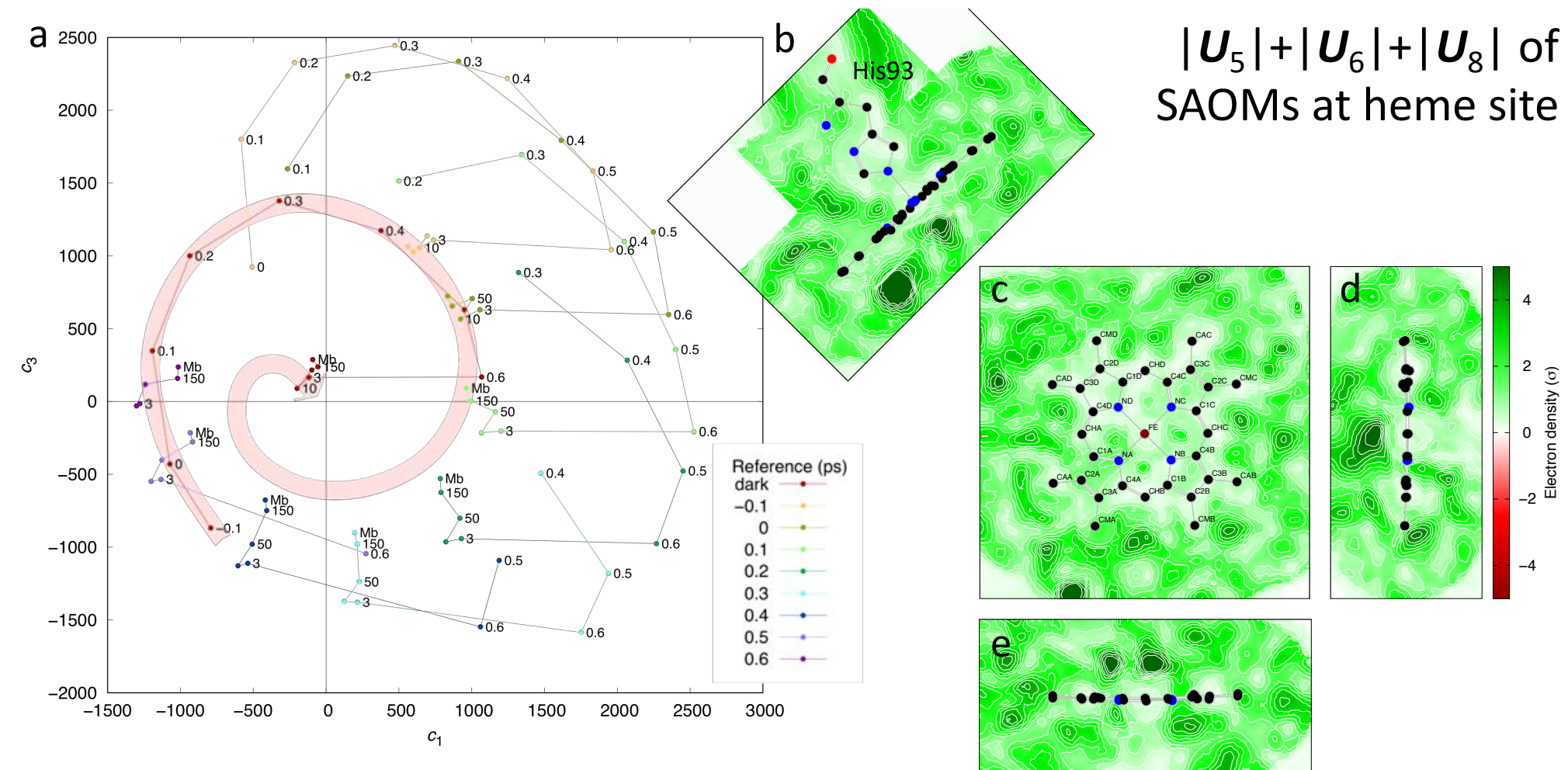

f

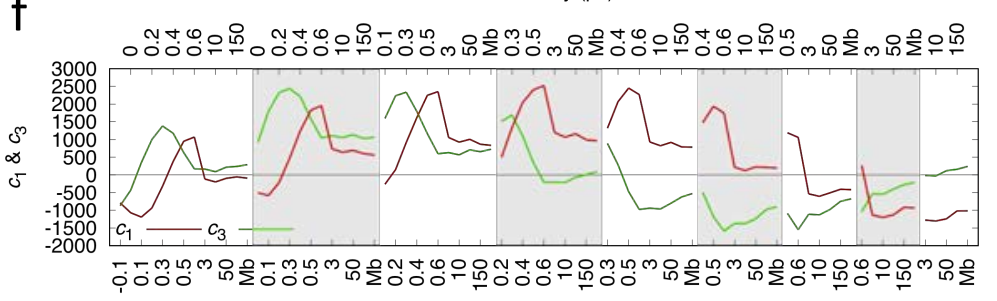

Time delay (ps)

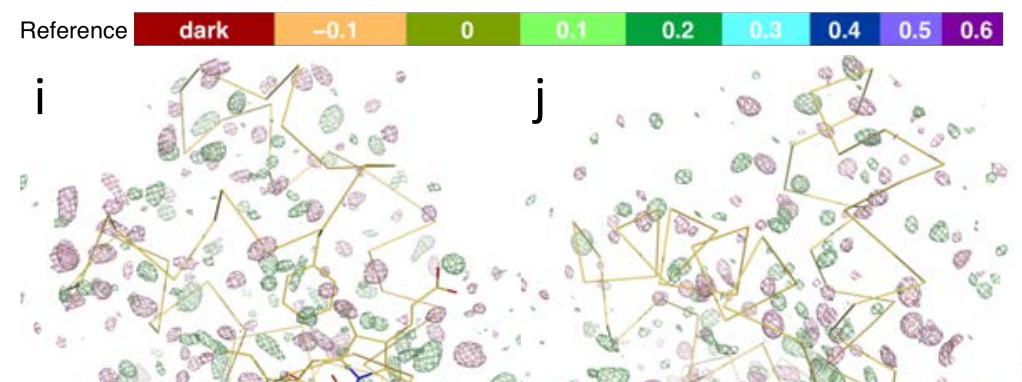

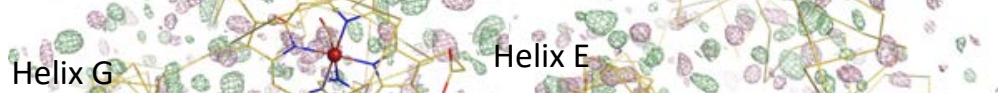
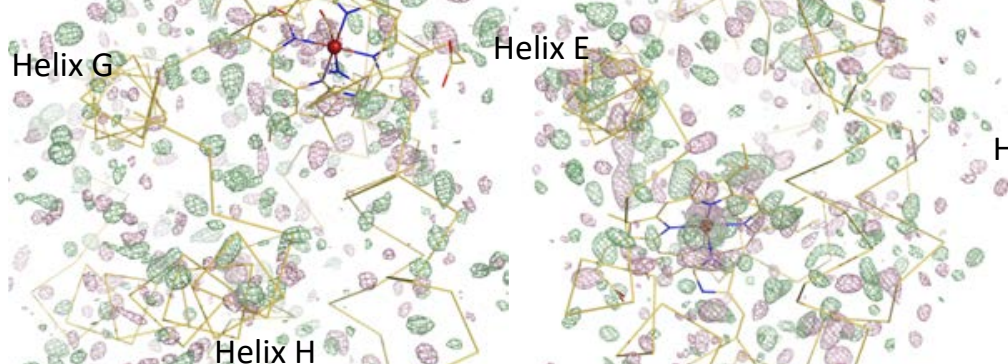

$\boldsymbol{U}_{1}$
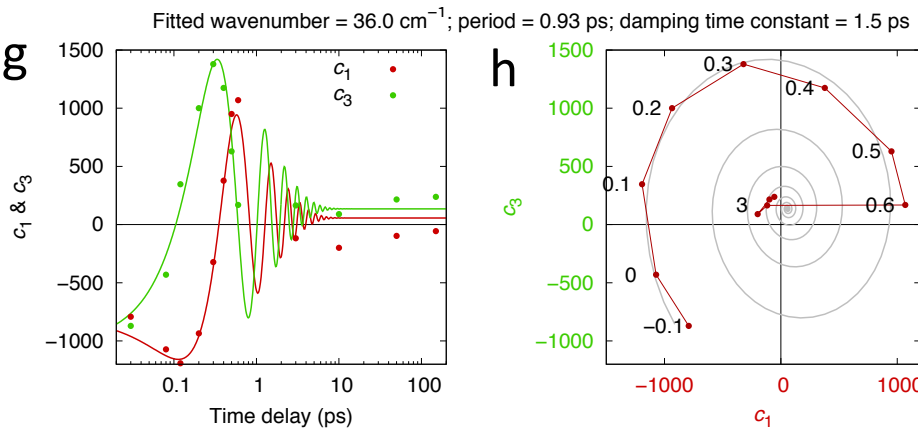

k

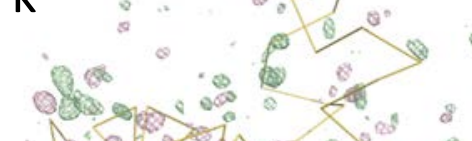

Helix E
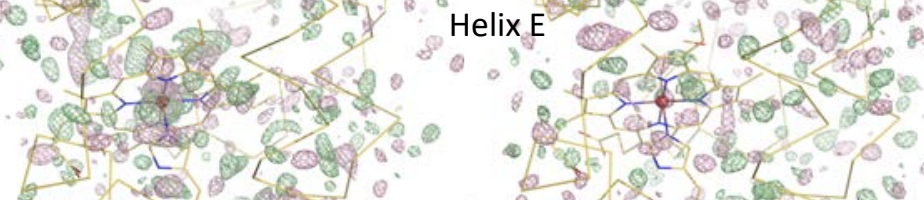

$U_{3}$

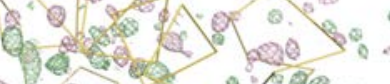

- $10.020 \%$

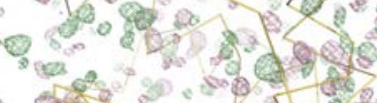

(18)

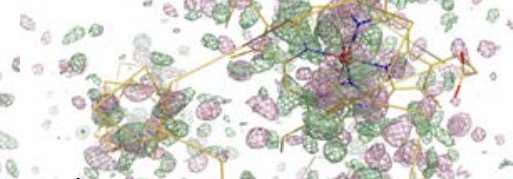

Helix G

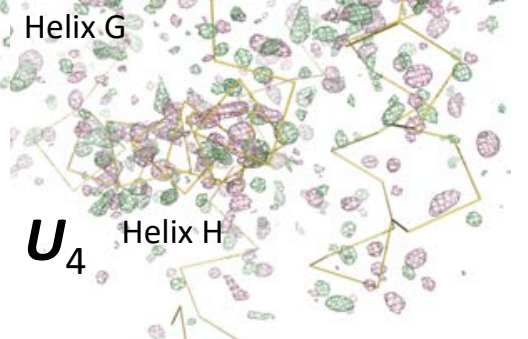

$\boldsymbol{U}_{1}$ through $\boldsymbol{U}_{4}$ of difference maps in the entire globin 
1121 Figure 7. Low frequency oscillations in the solvent. (a) SVD analysis of the

1122 difference maps in the entire globin produces the first and third components

1123 circularly oscillating before $1 \mathrm{ps}$ and quickly diminishing during the first few ps.

1124 Similar oscillations are also observed only around the heme site in both the

1125 difference maps (Fig. 2b) and the omit maps (Fig. 4b). (b-e) Cross sections

1126 through an averaged map of both the positive and negative halves of $\boldsymbol{U}_{5}, \boldsymbol{U}_{6}$, and

$1127 U_{8}$ of the SAOMs. Low densities in white and light green are located consistently

1128 on the heme and protein, which suggests that the oscillating signals are more

1129 associated with the solvent rather than the globin. (f) The circularly oscillating

1130 coefficients $c_{1}$ and $c_{3}$ are plotted as function of delay time in many series. The

1131 offset sinusoidal curves indicate a circular oscillation. Each map series is derived

1132 from subtracting a different reference dataset as indicated by the color bar below.

1133 ( $\mathrm{g}$ and $\mathrm{h}) c_{1}$ and $c_{3}$ of the $-F_{\text {dark }}$ series are least-squares fitted with a two-

1134 dimensional damped oscillation (Methods). (i-l) Comparison of several

1135 components of difference maps in the entire globin. $\boldsymbol{U}_{1}$ and $\boldsymbol{U}_{3}$ contain

1136 widespread signals, as they are ranked the most significant components. But no

1137 signal is concentrated on the heme or the protein. Instead, $\boldsymbol{U}_{2}$ and $\boldsymbol{U}_{4}$ clearly

1138 contain strong signals associated with the heme and secondary structures of the

1139 protein.

1140

1141 
Ultrafast Structural Changes Decomposed

1143 from Serial Crystallographic Data

1145 Zhong Ren

1146

1147 Department of Chemistry, University of Illinois at Chicago, Chicago, IL 60607, 1148 USA

1149 Renz Research, Inc., Westmont, IL 60559, USA

1151 zren@uic.edu

1153 Methods

1154 Difference Fourier maps

1155 Difference Fourier maps are commonly used in protein crystallography. A

1156 difference Fourier map is synthesized from a Fourier coefficient set of $F_{\text {light }}-F_{\text {reference }}$

1157 with the best available phase set. Before Fourier synthesis, $F_{\text {light }}$ and $F_{\text {reference }}$ must

1158 be properly scaled to the same level so that the distribution of difference values

1159 is centered at zero and not skewed either way. All available datasets from

1160 Barends \& Schlichting et al. (2015) are carefully scaled and difference Fourier

1161 maps are calculated using a software package dynami $X^{\mathrm{TM}}$ according to

1162 previously published protocols including a weighting scheme under the

1163 assumption that a greater amplitude of a difference Fourier coefficient $F_{\text {light- }}$

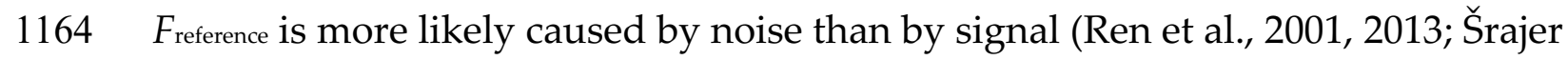

1165 et al., 2001; Ursby and Bourgeois, 1997).

Both the dark and light datasets can serve as a reference in difference maps.

1168 If a light dataset at a certain delay is chosen as a reference, the difference maps

1169 show the changes since that delay time but not the changes prior to that delay.

1170 These 91 maps in a full matrix provide independent observations for all changes

1171 (Table S1). This strategy is important to the subsequent singular value

1172 decomposition (SVD) analysis. In addition, two more difference maps of the

1173 photolyzed $\mathrm{Mb}^{*} \mathrm{CO}$ and ${ }^{* *} \mathrm{CO}(1 \mathrm{dws} / \mathrm{t})$ are also included in the $-F_{\text {dark }}$ series for

1174 SVD analysis. 
Each difference map is masked around the heme site. All densities at those grid points are set to zero, if they are greater than $5 \AA$ away from the heme, the bound or docked $\mathrm{CO}$, the side chain of the proximal His93, and that of the distal His64. Difference maps around the heme site include the most significant signals and exclude noises in the protein and solvent. However, the unmasked difference maps in the entire globin are also used a separate SVD analysis to

1182 include the protein and solvent regions for observation of global changes (Fig. 7i1183 1).

\section{Simulated annealing omit maps}

1186 The heme, the ligand, the side chains of proximal His93 and distal His64 are

1187 removed from all structures listed in Table S2. The remaining structures are

1188 refined using simulated annealing method with phenix.refine starting from a

1189 simulated temperature of 5,000 K. Fourier coefficients are obtained from the

1190 differences between the structure factor amplitudes $F_{\text {observed }}$ observed in the time-

1191 resolved experiments and the structure factor amplitudes $F_{\text {calculated calculated from }}$

1192 the omitted structures. The resulting $F_{\text {observed }}-F_{\text {calculated }}$ maps, known as simulated

1193 annealing omit maps (SAOMs), reveal the unbiased electron densities in the

1194 omitted region. Since the sperm whale (sw) Mb structures are in a different unit

1195 cell compared to the horse heart (hh) Mb structures, the SAOMs are aligned

1196 together according to a least-squares fitting of the main chain atoms in residues

1197 2-151. The heme and ligand are not used in the alignment procedure. Therefore,

1198 the motion of heme found in these omit maps is with respect to the globin

1199 framework (Fig. 5 and Movie S3). 20 aligned omit maps are subjected to the

1200 subsequent SVD analysis.

1201

1202 Singular value decomposition of (difference) electron density maps

1203 The principle of SVD can be found in texts on linear algebra. SVD analysis of

1204 (difference) electron density maps was previously described (Ren et al., 2013;

1205 Schmidt et al., 2003). Here I briefly recap this numerical procedure with an

1206 emphasis on the physical meaning of the outcome.

1207 
An electron density map, sometimes a difference map, consists of density values on a collection of grid points within a mask (see above), also known as voxels that are equivalent to pixels in a digitized image. All $M$ voxels in a threedimensional map can be serialized into a one-dimensional sequence of density values according to a specific protocol. It is not important what the protocol is as long as a consistent protocol is used to serialize all maps of the same grid setting and size, and a reverse protocol is available to erect a three-dimensional map from a sequence of $M$ densities. Therefore, a set of $N$ serialized maps, also known as vectors in linear algebra, can fill the columns of a data matrix $\mathbf{A}$ with no specific order so that the width of $\mathbf{A}$ is $N$ columns and the length is $M$ rows.

1218 Often, $M \gg>N$, thus $\mathbf{A}$ is an elongated matrix. In this work, $N=93$ for the difference maps and $N=20$ for the omit maps. If a consistent protocol of serialization is used, the corresponding voxel in all $N$ maps occupies a single row of matrix A. For example, the voxels at the heme iron in all maps could occupy the $j$ th row of matrix $\mathbf{A}$. This strict correspondence in a row of matrix $\mathbf{A}$ is important. Changes of the density values in a row are due to either signals, systematic errors, or noises.

SVD of the data matrix $\mathbf{A}$ results in $\mathbf{A}=\mathbf{U} \mathbf{W V}^{\mathrm{T}}$, also known as matrix factorization. Matrix $\mathbf{U}$ has the same shape as $\mathbf{A}$, that is, $N$ columns and $M$ rows.

1228 The $N$ columns contain decomposed basis components $\boldsymbol{U}_{k}$, known as left singular 1229 vectors of length $M$, where $k=1,2, \ldots, N$. Therefore, each component $\boldsymbol{U}_{k}$ can be 1230 erected using the reverse protocol to form a three-dimensional map. This

1231 decomposed elemental map can be presented in the same way as the original 1232 maps, for example, rendered in molecular graphics software such as Coot and

1233 PyMol. The second matrix $\mathbf{W}$ is a square matrix that contains all zeros except for

$1234 N$ positive values on its major diagonal, known as singular values $w$. The

1235 magnitude of $w_{k}$ is considered as a weight or significance of its corresponding

1236 component $\boldsymbol{U}_{k}$. The third matrix $\mathbf{V}$ is also a square matrix of $N \times N$. Each column

1237 of $\mathbf{V}$ or row of its transpose $\mathbf{V}^{\mathrm{T}}$, known as a right singular vector, contains the 1238 relative compositions of $\boldsymbol{U}_{k}$. That is to say, the $N$ values in a column $\boldsymbol{V}_{k}$ describe 1239 the relative compositions of the component $\boldsymbol{U}_{k}$ in the $N$ original maps. A singular 1240 triplet denotes 1 ) a decomposed component $\boldsymbol{U}_{k}, 2$ ) its singular value $w_{k}$, and 3) the 1241 composition function $V_{k}$. Singular triplets are often sorted in a descending order 
1242 of their singular values $w_{k}$. Only a small number of $n$ significant singular triplets

1243 identified by the greatest singular values $w_{1}$ through $w_{n}$ can be used in a linear

1244 combination to reconstitute a set of composite maps that closely resemble the

1245 original ones in matrix $\mathbf{A}$, where $n<N$. For example, the original map in the $i$ th

1246 column of matrix $\mathbf{A}$ can be closely represented by the $i$ th composite map $w_{1} v_{1 i} \boldsymbol{U}_{1}+$

$1247 w_{2} v_{2 i} \boldsymbol{U}_{2}+\ldots+w_{n} v_{n i} \boldsymbol{U}_{n}$, where $\left(v_{1 i}, v_{2 i}, \ldots\right)$ is the $i$ th row of matrix $\mathbf{V} . c_{k}=w_{k} v_{k i}$ in

1248 front of $\boldsymbol{U}_{k}$ is the coefficients for the linear combination. Excluding the

1249 components after $\boldsymbol{U}_{n}$ in this linear combination is based on an assumption that

1250 the singular values after $w_{n}$ are very small relative to those from $w_{1}$ through $w_{n}$.

1251 As a result, the structural information evenly distributed in all $N$ original maps is

1252 effectively concentrated into a far fewer number of $n$ significant components,

1253 known as information concentration or dimension reduction. In this study, the

1254 difference maps seem to contain 15 major components; the top nine are the most

1255 significant (Fig. 2e). The components 10 through 15 may very likely harbor less

1256 and less signals or systematic differences. For example, Fourier truncation

1257 errors, if significant enough, could occupy one or more of these components.

1258 However, the components 10 through 15 have not been examined in detail.

On the other hand, the trailing components in matrix $\mathbf{U}$ contain inconsistent

1261 fluctuations and random noises. Excluding these components effectively rejects

1262 noises. However, no clear boundary is guaranteed between signals, systematic

1263 errors, and noises. Systematic errors could be more significant than the desired

1264 signals, for example, the second and fourth components $\boldsymbol{U}_{2}$ and $\boldsymbol{U}_{4}$ of the omit

1265 maps describe significant differences between $\mathrm{hhMb}$ and $\mathrm{swMb}$, a valid scientific

1266 point to investigate but outside the scope of this paper.

1268 The orthonormal property of SVD

1269 The solution set of SVD must guarantee that the columns in $\mathbf{U}$ and $\mathbf{V}$, the left and

1270 right singular vectors $\boldsymbol{U}_{k}$ and $\boldsymbol{V}_{k}$, are orthonormal, that is, $\boldsymbol{U}_{h} \bullet \boldsymbol{U}_{k}=\boldsymbol{V}_{h} \bullet \boldsymbol{V}_{k}=0$

1271 (ortho) and $\boldsymbol{U}_{k} \bullet \boldsymbol{U}_{k}=\boldsymbol{V}_{k} \bullet \boldsymbol{V}_{k}=1$ (normal), where $h \neq k$ but both are from 1 to $N$. The

1272 orthonormal property also holds for the row vectors. As a result, each

1273 component $\boldsymbol{U}_{k}$ is independent of the other components. In other words, a

1274 component cannot be represented by a linear combination of any other

1275 components. For example, the symmetrical, spherical, and thermal component 
$1276 \boldsymbol{U}_{3}$ (Fig. 6) and the asymmetrical, cubic component of $\boldsymbol{U}_{7}$ (Fig. 5) of the omit maps

1277 are orthonormal. Both components show positive densities around the iron. But

1278 the cubic positive densities in $\boldsymbol{U}_{7}$ are associated with the strong negative densities

1279 on the ligand depicting dissociation, which is a direct link from ligand

1280 dissociation to regaining of the high-spin $3 d$ orbitals of the iron. On the other

1281 hand, no sign of ligand dissociation is found in the component $\boldsymbol{U}_{3}$ that features

1282 the spherical densities, which strongly suggests that this component describes

1283 the thermal effect. These two components are not interchangeable despite some

1284 similarity in the arrangement of positive densities, that is, one of the physical

1285 meanings of the orthonormal property of SVD. However, two physical or

1286 chemical parameters, such as temperature and $\mathrm{pH}$, may cause different changes

1287 to a structure, but not necessarily orthogonal changes (see below).

Due to the orthonormal property of SVD, an $\mathrm{N}$-dimensional Euclidean space is established, and the first $n$ dimensions define its most significant subspace.

1291 Each coefficient set $c_{k}=w_{k} v_{k i}$ of the $i$ th composite map is located in this subspace.

1292 All coefficient sets for $i=1,2, \ldots, N$ in different linear combinations to

1293 approximate the $N$ original maps in a least-squares sense can be represented by

$1294 N$ points in the $n$-dimensional Euclidean subspace. In this work, many two-

1295 dimensional orthographical projections of this $n$-dimensional subspace are

1296 presented as scatter plots with each map represented as a dot located at a

1297 position determined by the coefficient set of this map. These scatter plots are

1298 highly informative to reveal the relationship between the (difference) electron

1299 density maps and their metadata. For example, the difference maps at short time

1300 delays $<1$ ps are consistently different from those at long time delays $>1$ ps in a

1301 certain aspect (Fig. 2ac) and that two major components are circularly correlated

1302 but only before 1 ps, that is, a damped oscillation (Fig. 2b).

1304 Rotation in SVD space

1305 Dimension reduction is indeed effective in data analysis of dynamic

1306 crystallography when many datasets are evaluated at the same time. However,

1307 the interpretation of a basis component $\boldsymbol{U}_{k}$, that is, "what-does-it-mean", requires

1308 a clear demonstration of the relationship between the core data, here (difference)

1309 electron density maps, and their metadata, such as time, temperature, and other 
1310 experimental conditions. The outcome of SVD does not guarantee any physical 1311 meaning in a basis component. Therefore, SVD provides no direct answer to 1312 "what-does-it-mean". The factorized set of matrices $\mathbf{U}, \mathbf{W}$, and V from SVD is 1313 not a unique solution. That is to say, they are not the only solution to factorize 1314 matrix A. The default solution set from SVD may not carry an obvious physical 1315 meaning to depict "what-does-it-mean", thus its usefulness is very limited to 1316 merely a mathematical construction. A more straightforward analogy is that one 1317 can walk along two perpendicular streets to reach the opposite corner of a street 1318 block as if walking a diagonal block despite the fact that a diagonal street does 1319 not physically exist. This is also the origin of the debate on whether atomic 1320 orbitals as a basis set of mathematical constructions are physical and observable 1321 (Scerri, 2000). Therefore, it is very important to find one or more alternative 1322 solution sets that are physically meaningful to elucidate a structural 1323 interpretation. The concept of rotation after SVD was introduced by Henry \& 1324 Hofrichter (1992). But they suggested a protocol that fails to preserve the 1325 orthonormal and least-squares properties of SVD. The rotation process, also 1326 known as Givens rotation, is a connection between the analytical tool SVD and 1327 scientific findings. This rotation shall not be confused with a rotation in the 1328 three-dimensional real space, in which a molecular structure resides.

A rotation in the $n$-dimensional Euclidean subspace is necessary to change

1331 the perspective before a clear relationship emerges to elucidate scientific

1332 findings. It can be shown that two linear combinations are identical before and 1333 after a rotation of an angle $\theta$ in a two-dimensional subspace of $h$ and $k$. That is,

$$
{ }_{c h} \boldsymbol{U}_{h}+{ }_{c_{k}} \boldsymbol{U}_{k}=f_{h} \boldsymbol{R}_{h}+f_{k} \boldsymbol{R}_{k}
$$

1336 where $c_{h}$ and $c_{k}$ are the coefficients of the components $\boldsymbol{U}_{h}$ and $\boldsymbol{U}_{k}$ before the

1337 rotation as defined above; and $f_{h}$ and $f_{k}$ are the coefficients of the rotated basis

1338 components $\boldsymbol{R}_{h}$ and $\boldsymbol{R}_{k}$, respectively. The same Givens rotation is applied to both 1339 the bases and their coefficients:

1340

$$
\left\{\begin{array}{l}
\boldsymbol{R}_{h}=\boldsymbol{U}_{h} \cos \theta-\boldsymbol{U}_{k} \sin \theta \\
\boldsymbol{R}_{k}=\boldsymbol{U}_{h} \sin \theta+\boldsymbol{U}_{k} \cos \theta
\end{array}\right.
$$


Ren: Decomposition of ultrafast changes

1342 Obviously, the rotated components $\boldsymbol{R}_{h}$ and $\boldsymbol{R}_{k}$ remain mutually orthonormal and orthonormal to other components. And

$$
\left\{\begin{array}{l}
f_{h}=s_{h} t_{h}=c_{h} \cos \theta-c_{k} \sin \theta=w_{h} v_{h} \cos \theta-w_{k} v_{k} \sin \theta \\
f_{k}=s_{k} t_{k}=c_{h} \sin \theta+c_{k} \cos \theta=w_{h} v_{h} \sin \theta+w_{k} v_{k} \cos \theta
\end{array}\right.
$$

1346 Here $s_{h \mid k}=\sqrt{\sum f_{h \mid k}^{2}}$ are the singular values that replace $w_{h}$ and $w_{k}$, respectively,

1347 after the rotation. They may increase or decrease compared to the original

1348 singular values so that the descending order of the singular values no longer

1349 holds. $\boldsymbol{T}_{h \mid k}=\left(t_{h \mid k 1}, t_{h \mid k 2}, \ldots, t_{h \mid k N}\right)=\left(f_{h \mid k 1}, f_{h \mid k 2}, \ldots, f_{h \mid k N}\right) / s_{h \mid k}$ are the right singular

1350 vectors that replace $\boldsymbol{V}_{h}$ and $\boldsymbol{V}_{k}$, respectively. $\boldsymbol{T}_{h}$ and $\boldsymbol{T}_{k}$ remain mutually

1351 orthonormal after the rotation and orthonormal to other right singular vectors

1352 that are not involved in the rotation.

A rotation in two-dimensional subspace of $h$ and $k$ has no effect in other

1356 rotations can be carried out in many two-dimensional subspaces consecutively to

1357 achieve a multi-dimensional rotation. Rotation in the SVD space converts one

1358 solution set $\mathbf{A}=\mathbf{U W V}^{\mathrm{T}}$ to other alternative solutions $\mathbf{A}=\mathbf{R S T}^{\mathrm{T}}$ so that an

1359 appropriate perspective can be found to elucidate the relationship between the

1360 core data and metadata clearly and concisely. A solution derived from a rotation

1361 retains the orthonormal property of SVD. SVD analyses presented in this paper

1362 employ rotations extensively except that no distinction is made in the symbols of

1363 components and coefficients before and after a rotation.

1365 For example, a large gap between all difference maps $<1$ ps and those $>1$ ps 1366 indicates major differences between these two distinct time scales (Fig. 2a). A 1367 rotation does not change this fact except that this large gap used to orient along 1368 some inclined direction before the rotation. Therefore, the original component

$1369 U_{1}$ cannot describe the structural differences between the short delays $<1$ ps and 1370 the longer delays $>1$ ps, because the original $\boldsymbol{U}_{1}$ used to be a linear combination 1371 of several factors. The best rotation is to separate these two groups of difference 1372 maps along a major dimension, that is, a deterministic solution. The large gap 1373 exists as soon as an SVD analysis is performed and can be easily revealed in an 
1374 interactive three-dimensional plotting on the computer screen, although it is not

1375 obvious in any of the orthographical projections before a proper rotation is

1376 found. One cannot create nor eliminate this large gap by any rotation. A

1377 different rotation may reorient this large gap along another major dimension.

1378 But the structural conclusion would be equivalent.

\section{Least-squares fittings of oscillating components}

1395 maps. Usually, a pair of components are oscillating with the same frequency,

1396 which does not exclude the possibility that a third weaker component is also

1397 oscillating at the same frequency. A pair of coefficients in a two-dimensional

1398 damped oscillation of a period $p$ is least-squares fitted as $x$ and $y$ :

$$
\left\{\begin{array}{l}
x=x_{0}+a e^{-t / \tau} \sin (2 \pi t / p+\varphi) \\
y=y_{0}+b e^{-t / \tau} \sin (2 \pi t / p+\psi)
\end{array}\right.
$$

$1401 x_{0}$ and $y_{0}$ are the center of the oscillation. $a$ and $b$ are the amplitudes of the

1402 elliptical oscillation in two dimensions. They are damped according to an

1403 exponential decay with a time constant $\tau$. $\varphi$ and $\psi$ are the phase angles of the

1404 oscillation in two dimensions. They are not strictly $\pi / 2$ apart. 
Ren: Decomposition of ultrafast changes

1406 The sixth and eighth components of the difference maps appear to gain a

1407 two-dimensional oscillation after 1 ps (Fig. 2f). Their coefficients are least-

1408 squares fitted as $x$ and $y$ with an exponential onset:

1409

$$
\left\{\begin{array}{l}
x=x_{0}+a\left(1-e^{-t / \tau}\right) \cos (2 \pi t / p+\varphi) \\
y=y_{0}+a\left(1-e^{-t / \tau}\right) \sin (2 \pi t / p+\varphi)
\end{array}\right.
$$

1410

1411 Due to the lack of sufficient time points, the oscillation is forced to take a circular 1412 form.

1413

\section{Supplementary Table}

Table S1. The full matrix of difference Fourier maps

\begin{tabular}{|c|c|c|c|c|c|c|c|c|c|c|c|c|c|c|}
\hline Reference (ps) & Dark & \multicolumn{13}{|c|}{ Time points (ps) } \\
\hline${ }^{1}$ Dark $(5 \mathrm{cmv})$ & 2. & -0.1 & 0 & 0.1 & 0.2 & 0.3 & 0.4 & 0.5 & 0.6 & 3 & 10 & 50 & 150 & deoxy \\
\hline$-0.1(5 \mathrm{cn} 4)$ & $3-$ & . & 0 & 0.1 & 0.2 & 0.3 & 0.4 & 0.5 & 0.6 & 3 & 10 & 50 & 150 & deoxy \\
\hline $0(5 \mathrm{cn} 5)$ & - & - & . & 0.1 & 0.2 & 0.3 & 0.4 & 0.5 & 0.6 & 3 & 10 & 50 & 150 & deoxy \\
\hline $0.1(5 \mathrm{cn} 6)$ & - & - & - & . & 0.2 & 0.3 & 0.4 & 0.5 & 0.6 & 3 & 10 & 50 & 150 & deoxy \\
\hline $0.2(5 \mathrm{cn} 7)$ & - & - & - & - & . & 0.3 & 0.4 & 0.5 & 0.6 & 3 & 10 & 50 & 150 & deoxy \\
\hline $0.3(5 \mathrm{cn} 8)$ & - & - & - & - & - & . & 0.4 & 0.5 & 0.6 & 3 & 10 & 50 & 150 & deoxy \\
\hline $0.4(5 \mathrm{cn} 9)$ & - & - & - & - & - & - & . & 0.5 & 0.6 & 3 & 10 & 50 & 150 & deoxy \\
\hline $0.5(5 \mathrm{cnb})$ & - & - & - & - & - & - & - & $\cdot$ & 0.6 & 3 & 10 & 50 & 150 & deoxy \\
\hline 0.6 (5cnc) & - & - & - & - & - & - & - & - & . & 3 & 10 & 50 & 150 & deoxy \\
\hline 3 (5cnd) & - & - & - & - & - & - & - & - & - & . & 10 & 50 & 150 & deoxy \\
\hline 10 (5cne) & - & - & - & - & - & - & - & - & - & - & . & 50 & 150 & deoxy \\
\hline 50 (5cnf) & - & - & - & - & - & - & - & - & - & - & - & . & 150 & deoxy \\
\hline 150 (5cng) & - & - & - & - & - & - & - & - & - & - & - & - & . & deoxy \\
\hline Deoxy (5d5r) & - & - & - & - & - & - & - & - & - & - & - & - & - & \\
\hline PDB entry & $5 \mathrm{cmv}$ & $5 \mathrm{cn} 4$ & $5 \mathrm{cn} 5$ & $5 \mathrm{cn} 6$ & $5 \mathrm{cn} 7$ & $5 \mathrm{cn} 8$ & $5 \mathrm{cn} 9$ & $5 \mathrm{cnb}$ & $5 \mathrm{cnc}$ & $5 \mathrm{cnd}$ & 5 cne & $5 \mathrm{cnf}$ & $5 \mathrm{cng}$ & $5 \mathrm{~d} 5 \mathrm{r}$ \\
\hline
\end{tabular}

${ }^{1}$ Traditionally, the dark dataset is chosen as the reference in calculation of difference Fourier maps.

${ }^{2}$ Dot (.) indicates that these are empty maps.

${ }^{3}$ Dash (-) indicates that these are negated maps.

1415

1416 
Ren: Decomposition of ultrafast changes

\begin{tabular}{lll}
\hline \multicolumn{3}{l}{ Table S2. Dataset labeling } \\
\hline PDB entry & Label & Description \\
\hline $1 \mathrm{bz6}$ & aquomet & Aquomet Mb from sperm whale at room temperature \\
$1 \mathrm{bzp}$ & swMb & Deoxy Mb from sperm whale at room temperature \\
$1 \mathrm{bzr}$ & swMbCO & MbCO from sperm whale at room temperature \\
\hline $1 \mathrm{dwr}$ & $\mathrm{MbCO}$ & MbCO from horse heart at $100 \mathrm{~K}$ \\
$1 \mathrm{dws}$ & $\mathrm{Mb} \mathrm{CO}$ & Photolyzed MbCO from horse heart at $22 \mathrm{~K}$ \\
$1 \mathrm{dwt}$ & $\mathrm{Mb}$ * $\mathrm{CO}$ & Photorelaxed Mb from horse heart at $88 \mathrm{~K}$ \\
\hline $5 \mathrm{cmv}$ & dark & XFEL dark dataset of MbCO from horse heart \\
\hline $5 \mathrm{cn} 4$ & -0.1 & \\
$5 \mathrm{cn} 5$ & 0 & \\
$5 \mathrm{cn} 6$ & 0.1 & \\
$5 \mathrm{cn} 7$ & 0.2 & XFEL light datasets binned by delay time $<1$ ps \\
$5 \mathrm{cn} 8$ & 0.3 & \\
$5 \mathrm{cn} 9$ & 0.4 & \\
$5 \mathrm{cnb}$ & 0.5 & \\
$5 \mathrm{cnc}$ & 0.6 & \\
\hline $5 \mathrm{cnd}$ & 3 & \\
$5 \mathrm{cne}$ & 10 & XFEL light datasets with delay time $>1 \mathrm{ps}$ \\
$5 \mathrm{cnf}$ & 50 & \\
$5 \mathrm{cng}$ & 150 & Deoxy Mb from horse heart at $7^{\circ} \mathrm{C}$ \\
\hline $5 \mathrm{~d} 5 \mathrm{r}$ & $\mathrm{Mb}$ & \\
\hline & & \\
\hline
\end{tabular}




\section{Supplementary Figures and Legends}

1420

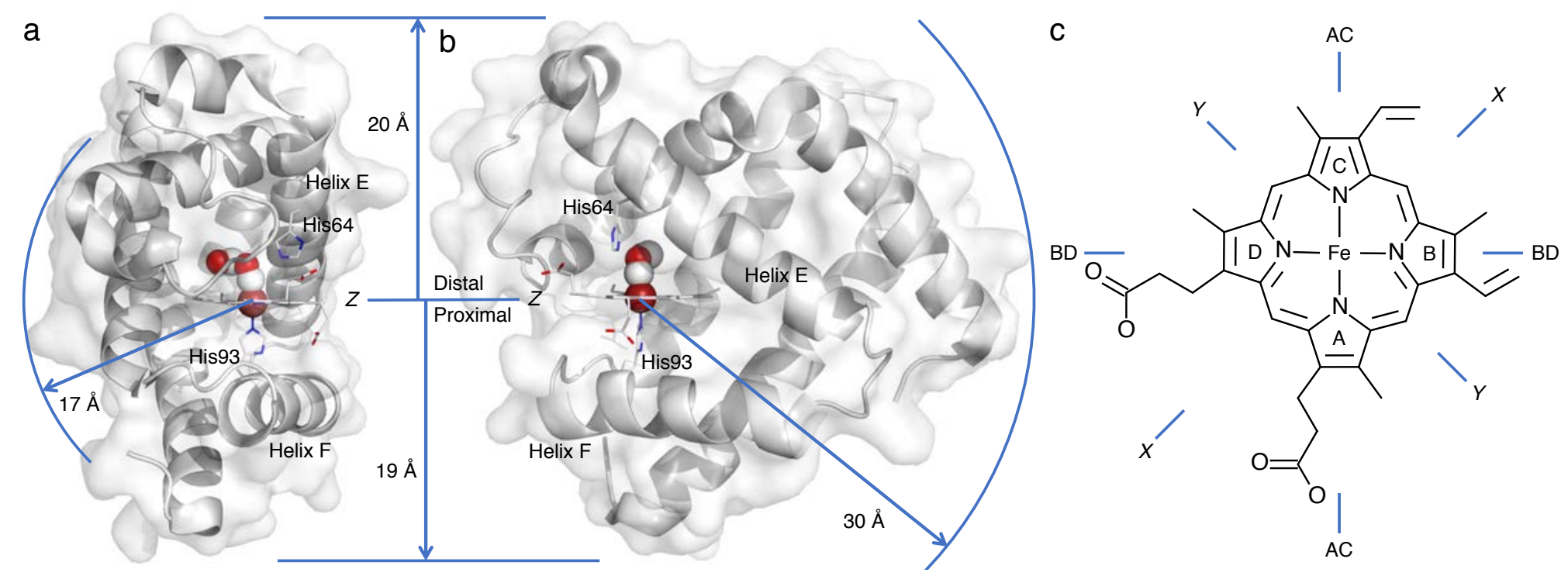

1422

1423 Figure S1. Orientations in myoglobin. Myoglobin $(\mathrm{Mb})$ is a $17 \mathrm{kDa}$ protein in

1424 muscle that carries molecular oxygen $\mathrm{O}_{2}$. The protein structure is rendered in

1425 ribbon and surface models in (a) and (b). The non-protein prosthetic group heme

1426 in stick model is anchored to helix F of the protein through His93 also in sticks.

1427 Two propionic acid side chains of the heme are facing the molecular surface. The

1428 sixth coordination site of the heme iron in sphere could bind $\mathrm{O}_{2}, \mathrm{CO}$, and $\mathrm{NO}$

1429 ligands also in spheres. Both a bound $\mathrm{CO}$ and a dissociated $\mathrm{CO}$ docked nearby

1430 are shown. Traditionally, the anchor side of the heme is called proximal with

1431 respect to the opposite distal side, where the ligands bind. The most remote part

1432 of the protein is $30 \AA$ away from the heme iron. The proximal and distal

1433 directions extend 19 and $20 \AA$ in the protein from the heme iron, respectively.

1434 The tetrapyrrole rings are traditionally named ABCD as marked in (c). In this

1435 paper, the heme plane is called $Z$ cross section. Other cross sections $X, Y, A C$,

1436 and BD are defined in (c).

1437 
bioRxiv preprint doi: https://doi.org/10.1101/634071; this version posted July 12,2019 . The copyright holder for this preprint (which was not certified by peer review) is the author/funder. All rights reserved. No reuse allowed without permission.

Ren: Decomposition of ultrafast changes
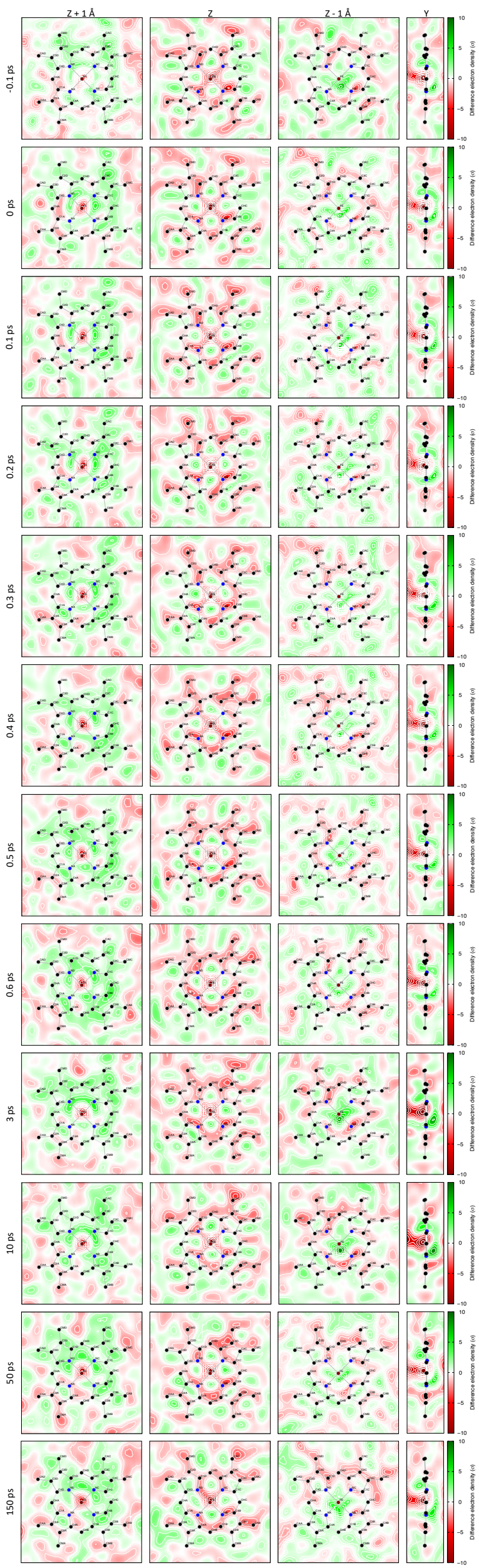
Ren: Decomposition of ultrafast changes

1439 Figure S2. The difference Fourier maps for SVD analysis. Difference maps are 1440 calculated according to the protocol described in Methods. Only the series 1441 from -0.1 to $150 \mathrm{ps}$ with the dark dataset $(5 \mathrm{cmv})$ as the reference is displayed 1442 here. Cross sections are marked on top. See the legends of Figs. S1 and 3 for 1443 definition of cross sections. See Table S1 for the full matrix of difference maps. A 1444 visual inspection of these difference maps at different delay times may conclude 1445 that they are largely identical. Very subtle variations could be seen by a careful 1446 comparison of these maps in a time series. A numerical deconvolution is 1447 necessary to extract the inherent components.

1448

1449 


\section{$\boldsymbol{U}_{1}$ of difference maps at} heme site

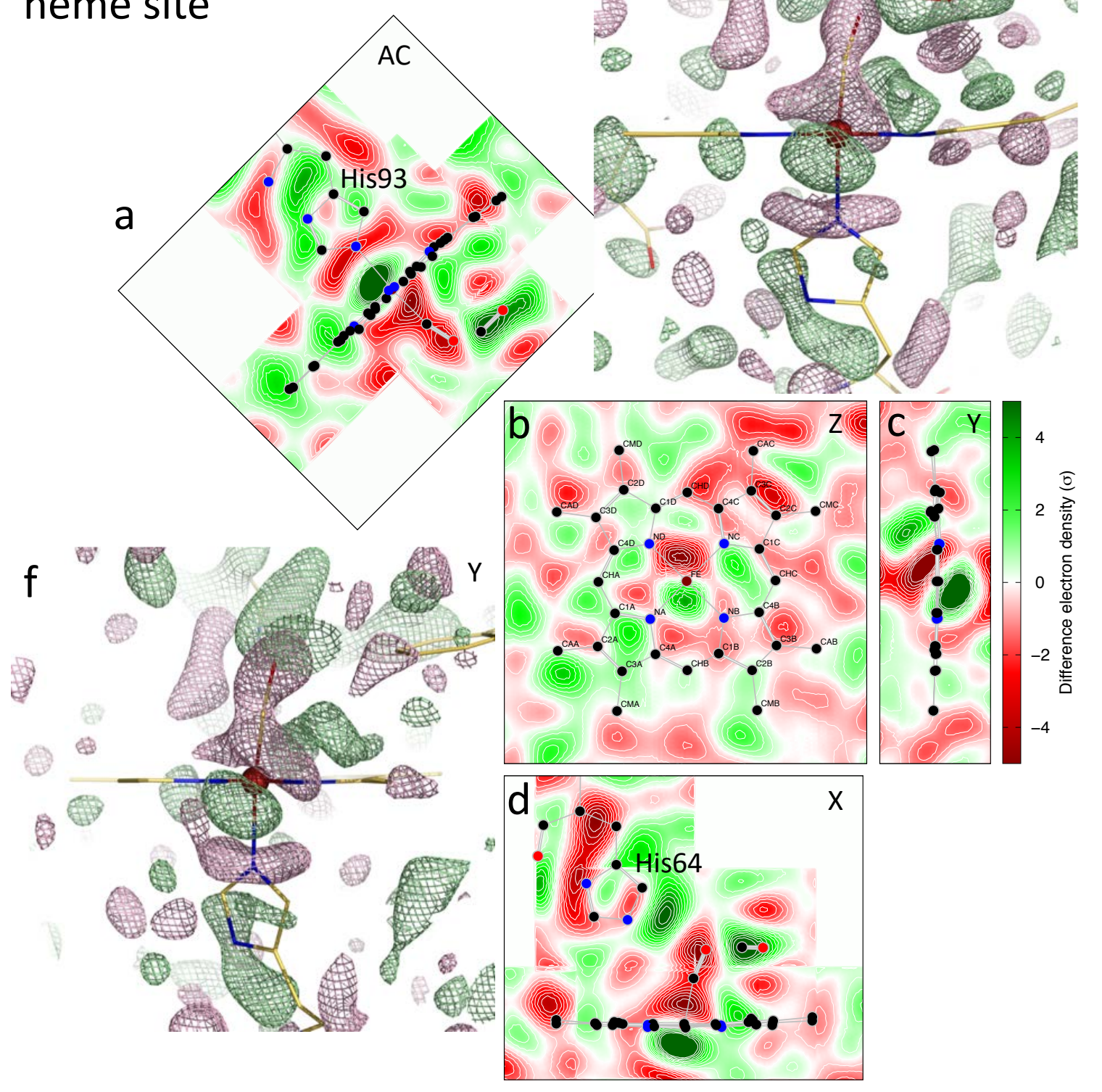

1452 Figure S3. The most significant component $\boldsymbol{U}_{1}$ of the difference maps. No doubt

1453 the most significant event shown in the difference maps is the out-of-plane

1454 displacement of the iron. This component shows the severely skewed iron

1455 displacement. The corresponding coefficient $c_{1}$ is plotted in Fig. 2ac. This

1456 component clearly separates the delays $>1$ ps from those $<1$ ps. (a-d) Images of

1457 two-dimensional cross sections through the first component. (e) and (f) Three-

1458 dimensional mesh contours of the component difference map. Green and red

1459 meshes are positive and negative contours at $\pm 7 \sigma$, respectively. 


\section{$\boldsymbol{U}_{6}$ of difference maps at heme site}
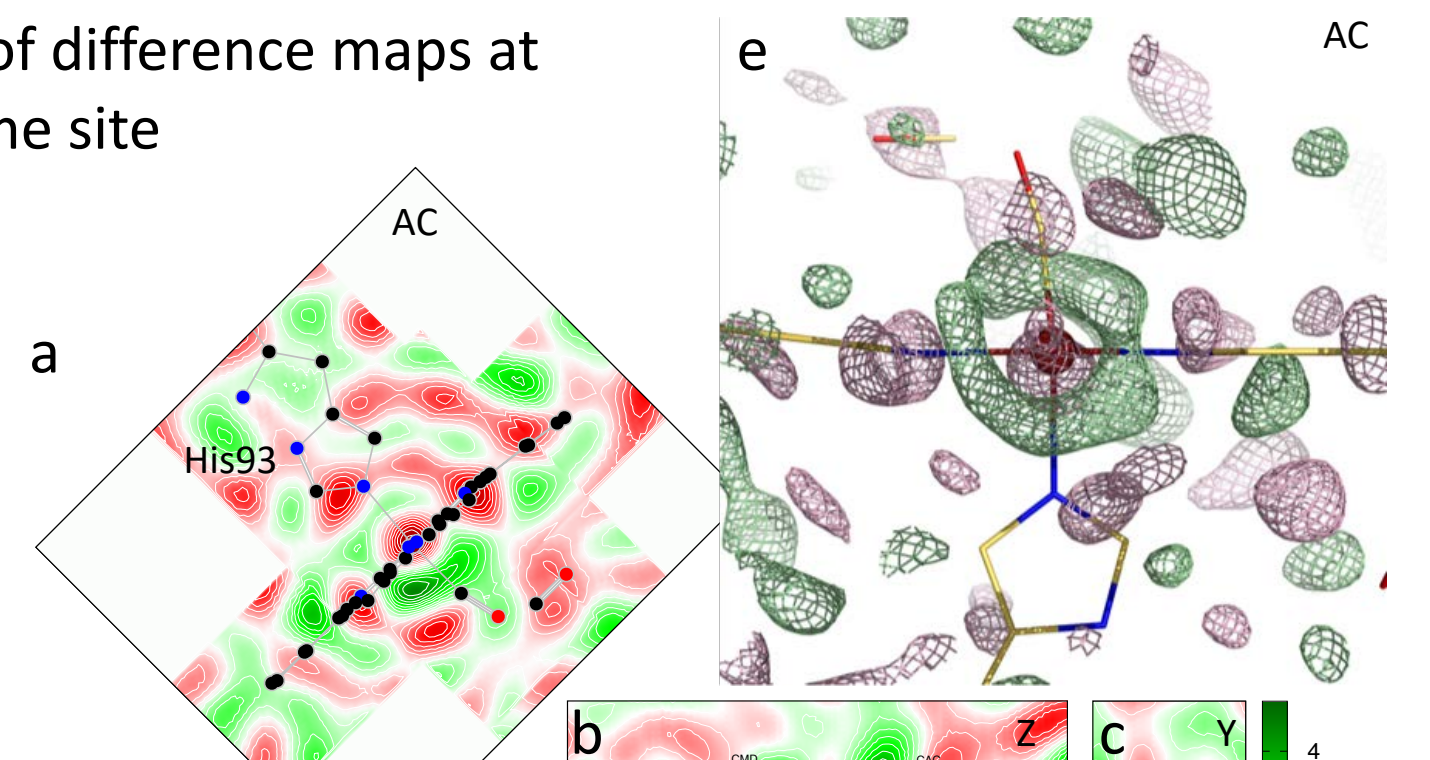

1462 Figure S4. Modification to the cubic positive densities around the iron in the

1463 sixth component $\boldsymbol{U}_{6}$ of the difference maps. The corresponding coefficient $c_{6}$ is

1464 plotted in Fig. 2f. This component and the eighth component $\boldsymbol{U}_{8}$ form a two-

1465 dimensional oscillation that ramps up in the delays $>1$ ps (Fig. $2 \mathrm{f}$ ). The effect of

1466 modulation and oscillation is shown in Movie S1. (a-d) Images of two-

1467 dimensional cross sections through the sixth component. (e) and (f) Three-

1468 dimensional mesh contours of the component difference map. Green and red

1469 meshes are positive and negative contours at $\pm 7 \sigma$, respectively. 
Ren: Decomposition of ultrafast changes
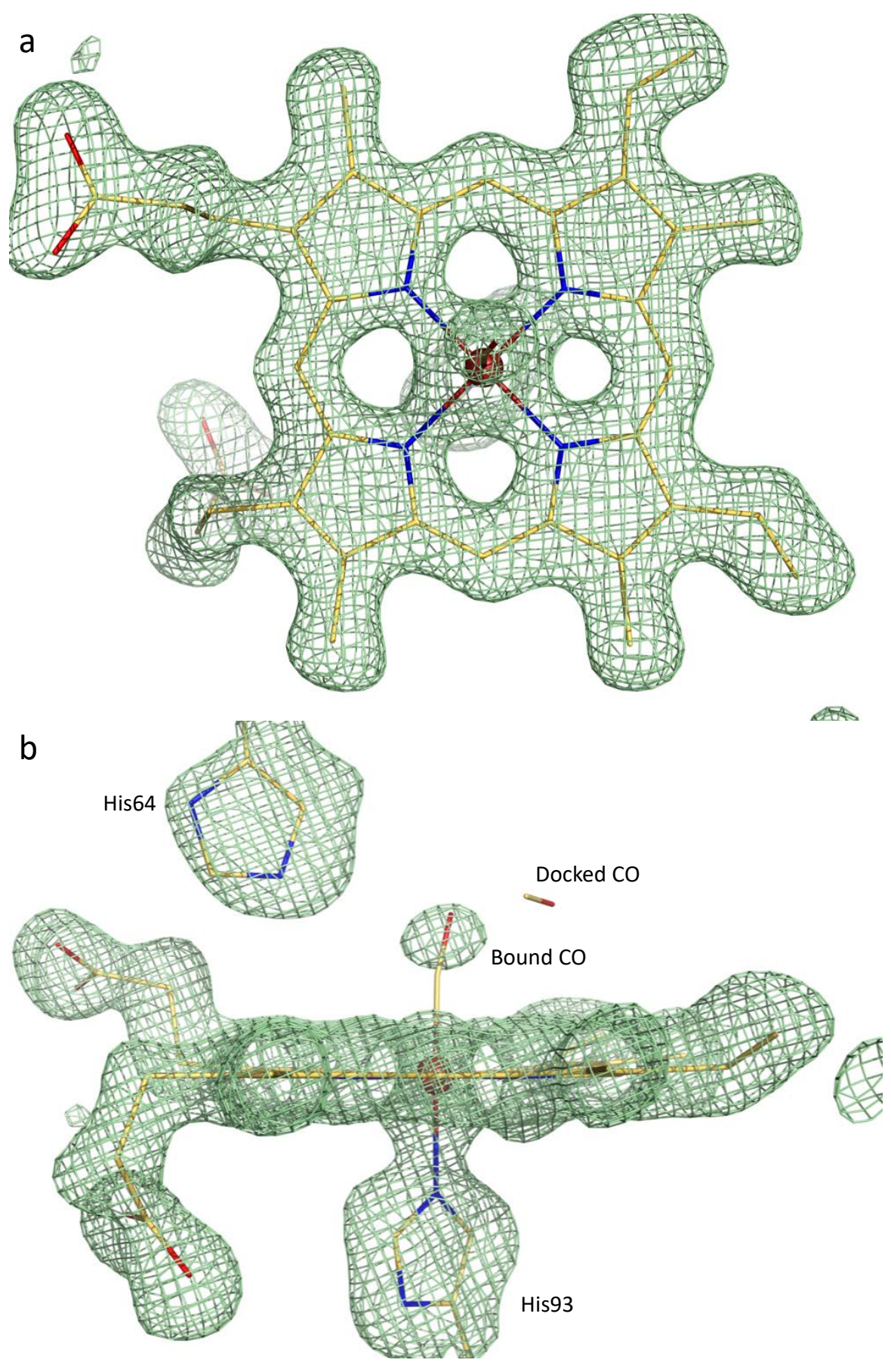

1472 Figure S5. The most significant component of SAOMs. Since an omit map is the 1473 least biased experimental electron density map, the most significant component 1474 of the omit maps, by far (Fig. 4f), is an averaged experimental electron density 1475 map of 20 omit maps. Only the ligand is not clear due to its variation. All other 1476 components will be added onto or subtracted from this average. 
Ren: Decomposition of ultrafast changes
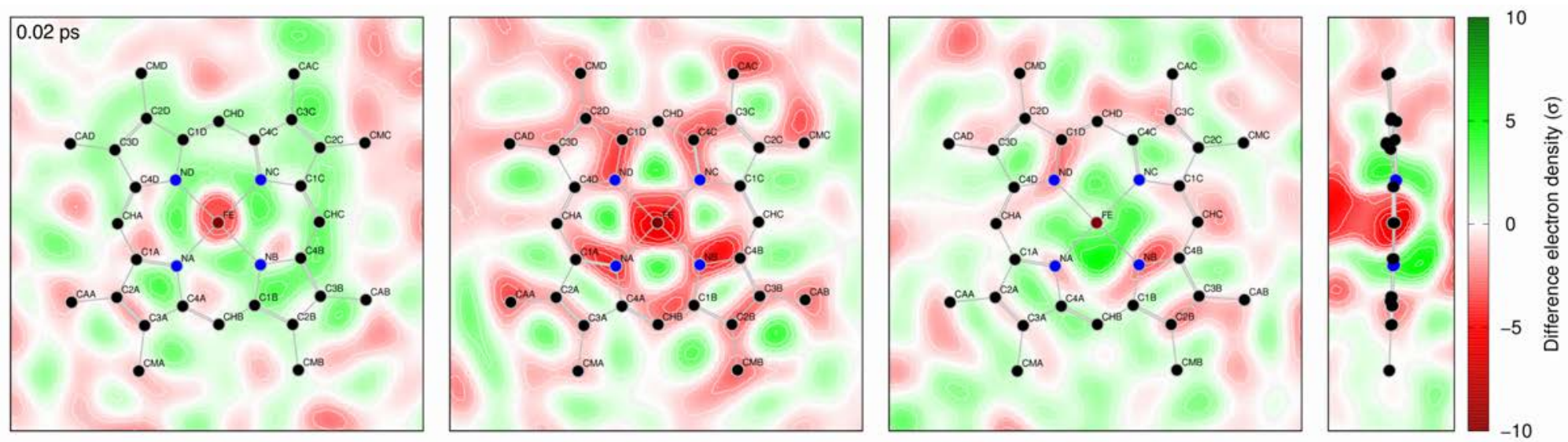

1479

1480 Movie S1. Reconstituted difference maps showing modulation and oscillation.

1481 Linear combination of components $\boldsymbol{U}_{1}, \boldsymbol{U}_{4}, \boldsymbol{U}_{6}$, and $\boldsymbol{U}_{8}$ of the difference maps span

1482 from 0.02 to 500 ps with some extrapolation on both ends. Other major

1483 components carrying systematic difference from the deoxy $\mathrm{Mb}$ or featuring

1484 oscillations in the solvent are not included in the linear combination to produce

1485 this mivie. The four panels are cross sections of $Z+1.1 \AA, Z, Z-1.1 \AA$, and $Y$,

1486 respectively. See Figs. S1 and 3 for the definition of cross sections. The cubic

1487 positive densities first lean towards ring $\mathrm{D}$ from 0.5 to $3 \mathrm{ps}$. Then the position

1488 returns back to the mean position after $50 \mathrm{ps}$, sways in the opposite direction

1489 towards ring $B$, and ramps up after 1 ps into an oscillation of a period of 220 ps

1490 (Fig. 2f). It is clear from this movie that the iron reaches its most out-of-plane

1491 displacement at a few ps and recoils back during 100-200 ps and pops out again

1492 after 200 ps.

1493

1494 
Ren: Decomposition of ultrafast changes
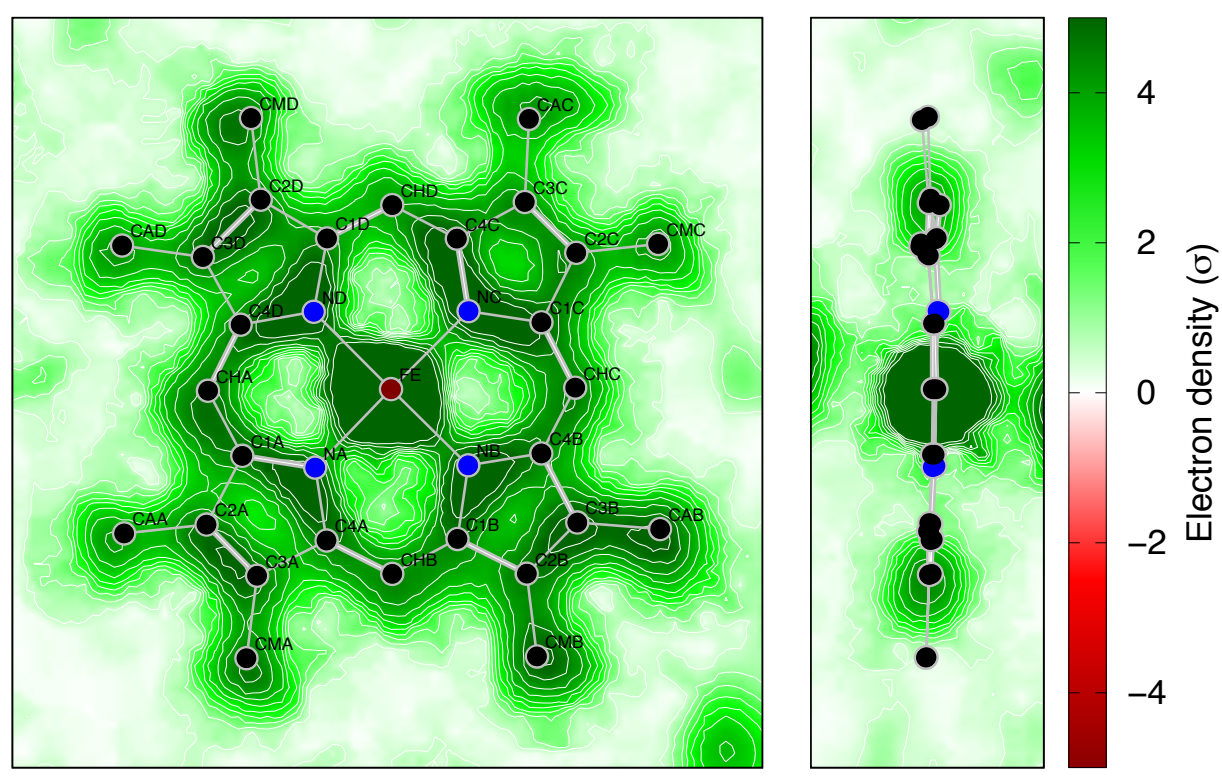

1497 Movie S2. Temperature effect on electron density map of the heme. The first and

1498 third components of the SAOMs are recombined to show the temperature effect

1499 only. The components related to the chemical signals of the photolysis are not

1500 included. Therefore, this movie shows the pure thermal effect. Slight

1501 extrapolation at both ends covers from 0 to $600 \mathrm{~K}$. The two panels are cross

1502 sections $Z$ and $Y$, respectively. See also Fig. $6 a b$ for electron density maps at 0

1503 and $600 \mathrm{~K}$. 

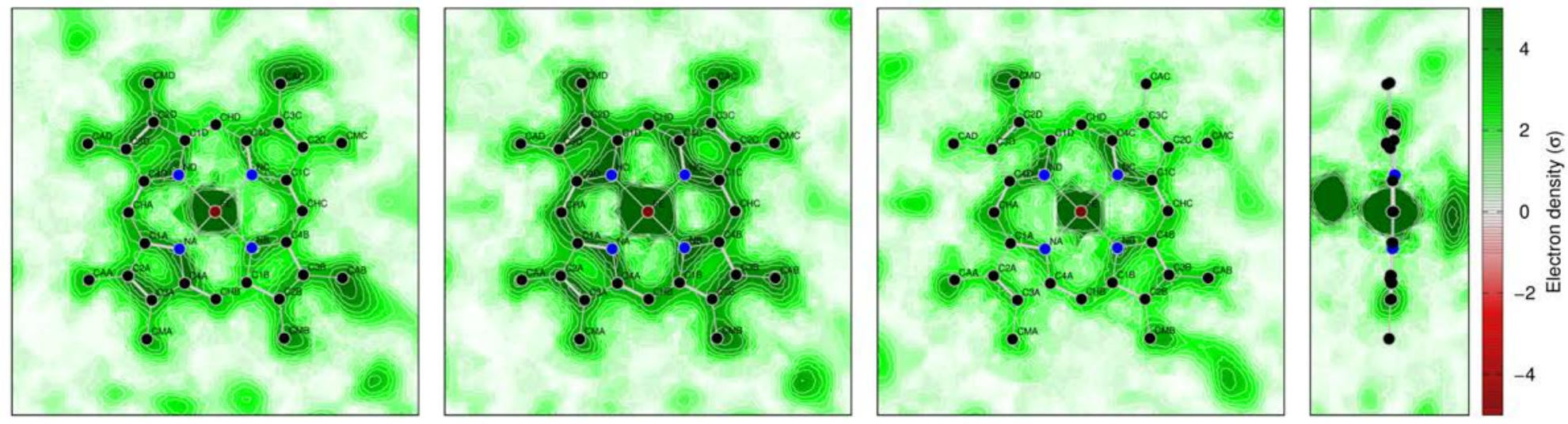

1507

1508 Movie S3. Comparison of electron density of the heme in ligated and unligated

1509 deoxy states. The first, third, and seventh components of the SAOMs are

1510 recombined to directly contrast the electron density maps of the heme in ligated

1511 and deoxy states. The coefficients for the first and third components are constant

1512 in the reconstituted maps at $0 \mathrm{~K}$, that is to say, the thermal effect is excluded.

1513 The coefficient $c 7$ for the seventh components is set to -400 for the ligated state

1514 and +300 for the deoxy state (Fig. 4e). The panels are cross sections of $Z+1.1 \AA, Z$,

$1515 Z-1.1 \AA$, and $Y$, respectively. The ligation state and the iron out-of-plane motion

1516 are shown in cross section $Y$. Cross section $Z+1.1 \AA$ on the distal side clearly

1517 shows greater densities of the heme in deoxy state, signaling the heme doming.

1518 Most importantly, the heme is sliding towards the AB rings in deoxy state. This

1519 is consistent with the skewed displacement of the iron and the motion of the

1520 proximal His93. 


\title{
Ultrafast Structural Changes Decomposed from Serial Crystallographic Data
}

\author{
Zhong Ren
}

Department of Chemistry, University of Illinois at Chicago, Chicago, IL 60607, USA Renz Research, Inc., Westmont, IL 60559, USA

zren@uic.edu

\section{Point-by-point responses to the reviewers' comments}

This manuscript has been extensively reviewed by two reviewers, both of whom evaluated the potential significance of the work and constructively suggested possible improvements. The reviewer 1 identified himself as Vladimir Y. Lunin of Russian Academy of Sciences. The reviewers' comments are copied below verbatim in italics followed by my response. The topics are arranged by relevance to one another. The complete comments are appended at the end.

Reviewer 1: The author challenges an ambitious task to extract tiny structural information possessing X-ray scattering data sets of a resolution only about $1.8 \AA$ while usually this requires data of much higher resolution, atomic 1.0-1.2 А or even ultrahigh, about $0.8 \AA$.

Reviewer 1: In particular, this leads to the fact that individual electrons and hydrogen atoms become distinguishable in Fourier difference syntheses only when working with data sets at resolution of 1.0-1.2 $\AA$ (atomic resolution), while partial charges (density of valence electrons) become visible only at about $0.8 \AA$ (ultra-high resolution) and only under certain restrictions on the structure refinement protocol (Afonine et al., 2004, Acta Cryst. D60, 260274).

Reviewer 2: C. Finally, some aspects of the central results could be presented with greater caution, considering that they are somewhat speculative, and that they depend on interpretation.

1) The author explains that the initial report of the observation of $3 d$ orbitals (Zuo el al 1999) was somewhat controversial. In particular Scerri (2000) states that orbitals of single electrons are fundamentally not observable, and recommends that observations be described 
in terms of electron density. This would be the most cautious language. However, haven't the philosophical arguments now run their course after 20 years, so it should now be safe to speak of the U4 component as revealing the high-spin orbitals? Unfortunately, the author's own description shows that the electron density is only marginally interpretable as a $3 d$ orbital. Line 368 states that the $3 d$ orbital for an isolated Fe(II) has 0.36 Angstrom radius, and line 369 that the orbital density in an Fe4S4 cluster has a maximum radius of 1 Angstrom. Meanwhile, the data shown here place the electron density at 1.7 to 1.9 Angstrom from the iron, depending on which map is considered, so it appears that this analysis is "first of its kind" result, rather than the observation of a $3 d$ orbital by established methods. It is somewhat difficult to understand the author's point of view in the following text (lines 372-394). It is stated that the cubic density is not the probability distribution of a $3 d$ electron, yet the paper states in many places that we observe a high-spin $3 d$ orbital.

Can crystallographic data at the resolution of $1.8 \AA$ reveal the electron density map of a single orbital? Clearly, this becomes one of the central questions to be addressed. I have consolidated previously scattered discussion into one new section in Discussion with a subtitle of "Are atomic orbitals observed?" (lines 635-710). This section should clarify my answer. A simple answer is No. However, the reasons behind this negative answer must be examined. More importantly, an accurate orbital density map of a single electron is not a goal in protein crystallography. These are my main points: First, the reason why an ultrahigh spatial resolution is required in charge-density analysis is because of the overwhelming spherical distribution of the inner electrons. Many techniques other than crystallography can capture images of atomic and molecular orbitals because these techniques are selectively sensitive to certain orbitals but not because they can achieve ultrahigh spatial resolution. If SVD is applied to chargedensity analysis, the requirement for spatial resolution will reduce. Sadly, my conversation with Philip Coppens cannot continue after his pass. Second, if there is no discontinuity in any probability distribution functions of electrons, why ultrahigh frequency terms are required in Fourier synthesis? There is probably no discontinuity in the first derivatives of these distributions either, which means no kinks. Ultrahigh spatial resolution is to describe discontinuities and kinks. Therefore, ultrahigh spatial resolution appears necessary more because of the demand to high accuracy of measurements and less because of the high spatial frequencies. Third, although difference Fourier technique has been used for decades, it did not ease the demand for 
ultrahigh spatial resolution because conformational changes brought even more complexity into the picture, which has prevented aspherical orbital densities from showing up. What makes the difference in revealing the remanence of individual orbitals at a given median resolution is the decomposition and deconvolution (continued below).

Reviewer 1: Creation of such tools would be a great step in development of X-ray research methods. Unfortunately, the results presented do not allow one to agree unconditionally with the author's conclusions about the progress achieved, as well as with the interpretation of the results obtained when processing previously published experimental data. In particular, the annotated author's conclusions on the visualization of the $3 d$ orbitals and structural rearrangements in the spin crossover process raise significant doubts. The suggestions and findings of the author could be a basis for further discussions, but the categorical style of interpretation of the results does not correspond to the level of evidence of these conclusions and may give rise to false illusions for non-experts in X-ray data processing. All this requires a substantial revision of the manuscript and of the conception. Reviewer 2: The author is completely correct (lines 237-239) to describe the literature-reported electron densities with unique shapes as being directly influenced by electrons occupying specific orbitals. However, there is a very large difference between the current diffraction dataset that diffracts to 1.8 Angstrom resolution, and the literature report of Hirano et al. (2016), showing iron $3 d$ and sulfur $3 p$ density at 0.48 Angstrom resolution. It is important to specifically compare the numerical resolution limits for the Hirano and the present work at both lines 236 and 370; in order to fully communicate that the present work is not simply a routine report of orbital density. Rather, the current work is lower-resolution and more speculative in nature.

It has been stated multiple times in the revised manuscript that the captured image is NOT an accurate depiction of the $3 d$ orbitals (lines 387-388 and 703-704), unlike the iron $3 d$ and sulfur $3 p$ orbitals at $0.48 \AA$. The difference in spatial resolution has been mentioned everywhere it can (lines 250 and 639). It is not a proper task for protein crystallography to provide accurate depictions of orbital densities. Instead, any direct evidence, at a good spatial resolution, to indicate a specific electronic event would be a desirable probe into the essence of biochemistry. This is one of the contributions of this work (continued below). 
Reviewer 2: The analysis leads to several astonishing claims highly relevant to myoglobin/CO binding, including that 1) the high-spin $3 d$ orbitals can be visualized throughout the time series, 2) the XFEL pulse heats up the specimen to $500 \mathrm{~K}$, and 3) there is an oscillation with a 220 ps period.

In addition to the visual evidence to indicate the reappearance of high-spin $3 d$ electrons, other conclusions such as the positional modulation, recoiling of the heme iron, the XFEL pulse induced temperature jump, and the low frequency oscillation in the solvent are as novel as the indication of the high-pin orbitals. These results are long awaited visual evidences to validate and to interpret decades of data in spectroscopy and computation. This work represents the highest achievement in serial femtosecond crystallography using XFELs. Each of the conclusions comes from a solid mathematical deduction of the XFEL datasets and corresponds to spectroscopic experiments and computational simulations in the field. However, making a reader "unconditionally" agree with my interpretations is beyond my paygrade.

Reviewer 1: Similarly, the author ignores that, when an electron transfers from one orbital to another, the image of the orbital in a difference map should appear twice: as a negative density of the orbital that has lost an electron, and a positive density of the orbitals that have gained an electron. He does not mention either the question on a principal possibility to see such a subtle change in 1.8 A resolution maps. The absence of the U4 component in the light-light series of difference maps is presented as evidence of the speed of this transition.

This is a very constructive suggestion. Indeed, it would be ideal to see some negative densities showing the loss of the low-spin electrons in the ligated state. However, the loss of the $\mathrm{CO}$ ligand and the drop of the iron cause the displacement of many more electrons than the low-spin electrons. Negative densities in the component $\boldsymbol{U}_{4}$ are overwhelming. At this resolution, the loss of the low-spin electrons cannot be separated from the main events upon photolysis. This note has been added (lines 400405).

Reviewer 2: Investigating the light-triggered release of $\mathrm{CO}$ from myoglobin, the author collectively re-examines electron density maps from room temperature and cryo-trapped 
structures, as well as the time-dependent XFEL paper [Barends et al. (2015)] covering the 0.1 ps to 150 ps time evolution. Mutual difference maps and single-dataset omit maps are analyzed with the Schmidt et al. (2003) method of singular value decomposition (SVD), to identify features in the electron density that change with either time, sample, or temperature. This reviewer does not know if SVD has ever been applied over so many independent variables, and if so it is worth mentioning.

Reviewer 2: 3) It is further stated (lines 390-393) that normal 2Fo-Fc maps cannot reveal small orbital changes, but that SVD analysis is highly sensitive. If true, this would be a critical factor, and everyone should be using SVD to analyze structure comparisons. It would be highly beneficial to lay out the evidence and controls, or provide literature references.

Applications of SVD in protein crystallography have had limited success in the past 20 years until Givens rotations are applied, mainly by me (Ren PLoS One 8, e77141 \& e77363, 2013; Ren Nucleic Acids Res 6, 1, 2016). This is due to the fact that the default solution of SVD guarantees no physical meaning in the basis components. Instead, each component is a linear combination or weighted sum of several meaningful images. SVD guarantees all basis components are orthonormal. However, physically meaningful images are not necessarily orthonormal. Therefore, a satisfactory interpretation of an SVD result is based on luck, which is the "drawback" of SVD.

Reviewer 1: An essential drawback of the suggested approach is the stage of the "manual" rotation of the found set of basic density distributions with unclear criteria of the best choice. This makes it difficult to reproduce the presented results.

Reviewer 1: The author's approach to the study of a series of experimental electron density distributions close to each other is based on a representation of each of these distributions as a weighted sum of a fixed set of density distributions pairwise uncorrelated, considered as a set of basis vectors mutually orthogonal. A visual study of the evolution of electron density under changing of external conditions is substituted by a numeric analysis of the weights with which these basis functions enter into one or another experimental density distribution. Sets of basic vectors can be selected in many ways and the basic distributions themselves may have no structural meaning. The recommendation of the author is to choose the basis in which "... an appropriate perspective can be found to elucidate the relationship between the core data and metadata clearly and concisely". This recommendation is extremely vague, 
opening up the scope for the selection of the "perspective" that confirms most the hypothesis of the researcher, and makes it difficult to reproduce and validate the results obtained. Reviewer 2: 4) The left-singular vectors are not unique - this is correctly described in the supplementary material starting at line 1201. At 1249 it is stated that a Givens rotation is applied so that an appropriate perspective can be found to elucidate the relationship between the core data and metadata. However, this leaves us with a situation where the main results of the paper (e.g., the U4 difference map showing high-spin density) are not based on a deterministic procedure! To paraphrase what I understand from this description, U4 was chosen by manually finding a Givens rotation that most clearly correlates with timedependence. However, the author does not give a protocol to find that rotation, nor a metric to judge when the rotation is optimized.

The concept of rotation was introduced much earlier by Henry \& Hofrichter (in Num Comp Meth 210, 129, 1992). But their protocol specializes in the field of spectroscopy. More importantly, it fails to preserve the orthonormal and least-squares properties of SVD after rotation. This manuscript is the first time that I explicitly describe a rotation procedure with the orthonormal and least-squares properties preserved. That is to say, the rotation procedure finds an alternative solution for SVD. The rotation does not change the internal relationship among datasets except that it finds a different perspective to view the relationship. This rotation procedure may require a user decision. But this is not a drawback; this is a major advance in data analysis of dynamic crystallography. It is this rotation that finally connects the core crystallographic data to the metadata of experimental conditions. This rotation finally accomplishes the deconvolution of physical or chemical factors after decomposition. It is absolutely true that "everyone should be using SVD to analyze structure comparisons." I also believe that this advance is not limited to crystallography. It is going to impact other fields using SVD.

For example in Fig. 2a, there is a large gap between all datasets $<1$ ps and those $>1$ ps, where "large" is with respect to the spacings among the datasets in each group. A rotation does not change this fact except that this gap was not originally along the first dimension $c_{1}$. Therefore, the original $\boldsymbol{U}_{1}$ cannot describe the structural meaning of this gap, that is, the difference between the short delays $<1$ ps and the longer delays $>1$ ps, because the original $\boldsymbol{U}_{1}$ used to be a linear combination of several factors. The criteria of 
the best choice are clear, that is, to separate these two groups along a major dimension, a deterministic solution. However, did I know this large gap before SVD? Of course not. This is where a user input is necessary. Nevertheless, one cannot create this large gap by any rotation. It always exists even I don't see it before a proper rotation is found. In fact, an interactive 3D plotting on the computer screen easily shows this large gap. What if another person decides to rotate in a different way? It is entirely possible that this large gap ends up orienting along another dimension. But the conclusion would not change. What if one decides not to apply any rotation? A linear combination of several components can still explain the inclined large gap with an identical conclusion.

For another example in Fig. $2 \mathrm{~cd}$, all difference maps have $c_{4} \approx 0$ except those calculated with the dark dataset as a reference, the red trace. The criteria of the best rotation are very clear and deterministic so that $\boldsymbol{U}_{4}$ depicts features only in the difference maps with the dark dataset as a reference. Again, the fact cannot be changed with any rotation that light-dark difference maps standout from the other maps except that the other maps were not originally on the plane of $c_{4}=0$. Therefore, the original $\boldsymbol{U}_{4}$ was puzzling with several physical meanings fixed in it. In this sense, it can be stated that the Ren rotation protocol completes a deconvolution of physical and chemical factors after a singular value decomposition. A more concise version of these discussions has been incorporated into Methods (lines 1365-1391).

Reviewer 2: If I were to perform an SVD analysis on a new protein system, how would I go about applying the Ren methodology to this new system? The core issue here is reproducibility - it would be good if more details were given about how the rotations were derived, for this particular work. Would the conclusions have been robust if slightly different U-vectors were chosen?

A satisfactory interpretation in an SVD analysis, the rotation after SVD in particular, highly depends on the scientific question. A major basis component may very likely describe an uninteresting fact that has been known for many years, the so-called trivial component. A major basis may also describe a systematic error, interesting but offtopic. The key to a proper rotation is the correlation between the core data and metadata, the reason why the name meta-analysis (Ren et al. PNAS 109, 107, 2012). 
With a clear scientific question in mind, a rotation is deterministic. However, before the data are evaluated by SVD, it is indeed difficult to predict what SVD may turn out. Since two different physical parameters, say temperature and $\mathrm{pH}$, may not cause orthogonal changes to a structure, a single rotation is not sufficient to satisfy both dimensions. That is to say, multiple rotations, thus multiple perspectives, are needed to answer all the scientific questions. If two parameters are largely orthogonal to each other, the solutions are deterministic and stable. However, if two parameters are highly colinear, that is, they cause very similar structural responses, the solutions could be less stable. Discussion and Methods have been expanded. However, this is not a paper on applications of SVD.

Reviewer 1: A key problem in identifying small changes in the electron density of an object in a series of experiments under variable conditions is that one never has the true distribution of electron density. What a researcher has instead is a Fourier synthesis of electron density, always of a limited resolution. Saying nothing about experimental errors, these syntheses contain larger or smaller distortion, caused by lack of the necessary data. The magnitude of such distortions, called Fourier series truncation errors, can be significant and these distortions can mask the subtle changes in the electron density.

Reviewer 1: Even 0.6 A-resolution syntheses can contain Fourier series truncation errors exceeding the signal level and mimicking structural details. The results presented by the author have been obtained at resolution of $1.8 \AA$, which is intermediate between "medium" and "high" resolutions according to the existing crystallographic classification. At such conditions, sufficiently large distortions of Fourier syntheses are expected. In this regard, the questions arise: "What are the changes studied in the manuscript? Are they those in the electronic structure of the sample or just a game of the Fourier series truncation errors?" The author does not pay any attention to these circumstances. Moreover, the author regularly speaks of "ultrahigh resolution", which can confuse the reader.

The topic of Fourier truncation error is not new. Its impact to electron density maps has been well understood. Errors from this source are not an unpredictable ghost. Therefore, we cannot attribute everything that we don't understand to this error. First, a Fourier truncation error may most likely occur to several static datasets included in this analysis, in which the spatial resolutions are truncated to $1.75 \AA$ to match that of XFEL datasets. However, such errors are not clearly present in these datasets, thanks to 
the difference Fourier technique and the simulated annealing protocol. Second, when the average intensity of an experimental dataset naturally decays as function of the spatial resolution, Fourier truncation errors are damped to a minimum. A low resolution dataset not necessarily contains more severe Fourier truncation errors; it simply lacks more details. Third, difference Fourier technique again self-cancels the remaining errors because both datasets would carry similar Fourier truncation errors. Fourth, the applied weighting scheme severely reduces any large differences in Fourier synthesis (Methods), which also prevents Fourier truncation errors. Fifth, even when Fourier truncation errors occur, such errors may appear as minor components after SVD (Figs. 2e and 4f), therefore isolated from the signals under discussion. Sixth, the SVD rotations validate that the signals under discussion, such as the positive cubic network, is not due to Fourier truncation errors because of its correlation with the metadata. An artifact due to Fourier truncation errors, if so conclude, needs to be explained why it is correlated with a physical or chemical parameter. As a conclusion, Fourier truncation errors are well guarded from. New findings cannot be dismissed under the fear of such errors. Discussion on Fourier truncation errors has been added (lines 679-695).

Reviewer 1: The start data is a set of several series of difference maps. The first series is based on the differences between results of the "dark" (without the use of exciting light pulses) experiment and ones in which the crystals were irradiated both light X-ray pulses ("light" sets), with different time delay

$F \_i \wedge l i g h t-k \_(i, 0) \cdot F_{-} \wedge$ dark $, i=1,2,3, \ldots$

The remaining difference maps were calculated using only "light" data sets $F_{-} i^{\wedge}$ light-k_(i,j).F_j^light $, i=j, j+1, \ldots$ Here $k_{-}(i, j)$ are scaling factors, individual for each map.

I start with a naive crystallographic explanation of the results. The first series of maps is shown in Fig. S2. These maps do not reproduce the typical pattern of difference maps, but rather reproduce a flipped (the one with the sign changed) image of the electron density distribution in the vicinity of the heme (the initial distribution is shown in Fig. S5). This is apparently caused by a imbalance in the difference syntheses of the first series, i.e. by too large values of the coefficients $k_{-}(i, 0)$, that results in the $-F^{\wedge}$ dark component dominating the maps. 
It remains very puzzling to me after many readings of the above comments that Lunin, the first reviewer, calls the difference maps displayed in Fig. S2 "not typical" and "flipped sign". There are two ways to interpret Lunin's comments: The positive and negative densities in the difference maps are mistakenly flipped or the difference maps look like a negation of an electron density map in Fig. S5. Neither makes sense to me. The convention in producing these difference maps is light-dark so that positive densities show the product of light and negative densities show the loss of the dark state. The first column of Fig. S2 Z+1 $\mathrm{A}$ shows a cross section parallel to the heme plane and $1 \AA$ towards to distal side. The second column $Z$ shows the heme plane. It is obvious that $Z+1 \AA$ cross section has more positive densities and $Z$ cross section has more negative densities. This is a typical difference map of light-dark at the heme site and cannot have a flipped sign because the heme buckles towards the distal side as a result of the light illumination. Also, the bound CO ligand is in an intense negative peak indicating the loss of the ligand and an intense positive peak skewed on the proximal side of the iron (right column in Fig. S2). Again, this is typical and without sign flipping. These maps are identical to those originally produced by Barends \& Schlichting (Science 350, 445, 2015) except in an arguably better quality. The scaling of the light and dark datasets is perfectly balanced as judged by equal amounts of positive and negative densities. Alternatively, a sign flipped image from Fig. S5 in Fig. S2? Of course, this is light-dark, the part of -dark should be a sign flipped image (see below). It seems that there exist some fundamental misunderstandings I have not figured out.

Reviewer 1: After decomposition, a balanced differential density distribution appears as the basic U1 distribution (Fig. S3). This map is close to the difference map of $1.8 \AA$ resolution. The excessive presence of the term $-F^{\wedge}$ dark in the initial difference syntheses $F^{\wedge}$ light- $\measuredangle k \cdot F \Omega$ $\wedge$ dark is compensated by U4 component (Fig. 3c), which resembles again the flipped image of density in the vicinity of the heme (Fig. S5). As noted by the author, in light-to-light difference syntheses $F_{-} i^{\wedge}$ light $k \cdot F_{-} j^{\wedge}$ light the U4 component is absent. This can be explained by a better balance of syntheses coefficients when working with data sets obtained under the same experimental conditions (in the presence of both "light" and "dark" X-ray pulses) and, as a result, the absence of a "ghost" corresponding to the initial heme density distribution.

Essentially, Lunin questions a possibility that the light-dark series is not scaled as well as the other light-light series, because the light and dark datasets were obtained 
under slightly different conditions, such as no laser pulse for the dark dataset. As a result, a "ghost" component exists in the light-dark series but not in the light-light series that has been decomposed by SVD as $\boldsymbol{U}_{4}$. The principle in meta-analysis is the correlation between core data and metadata. If the component $\boldsymbol{U}_{4}$ is an artifact due to scaling errors, it must be explained why these errors remain constant for short delays < 1 ps and decay as function of time $>1$ ps. The most significant systematic error should occur to the deoxy dataset collected at a synchrotron instead of an XFEL (marked as Mb in Fig. 2). However, this dataset fits smoothly into the trace. Again, an unusual result cannot be simply dismissed by an unexplained "error", here a scaling error.

Reviewer 1: The author suggests a totally different explanation declaring U4 component as visualization of $3 d$ orbital. An attention is not paid to the similarity of this distribution with the density distributions in the vicinity of the heme, shown in Fig. S2 and S5, as well as to the discrepancy between the size of the orbitals and the size of the density fragments in the U4 component noted by the author.

Again, this comment is very puzzling to me. There is a negative image of Fig. S5 in Fig. S2. This is correct, since Fig. S2 shows light-dark difference maps and Fig. S5 shows an average map of all datasets, which is very close to the dark map. The negative part of Fig. S2 is supposed to be similar to Fig. S5. As the size of the density features, there is not enough spatial resolution to depict an accurate map of the $3 d$ orbitals (see above). However, this is not to say that the net gain of densities further away from the iron is completely meaningless. The shape of the net gain coincides with the calculated orbital densities, which strongly suggests the influence of the high-spin $3 d$ orbitals.

Reviewer 2: 3) It is difficult to understand why the time evolution in Fig. $2 f$ is described as an "oscillation". Not even one full cycle is observed, so it should not be assumed to be a circular progression. If there were more time points available they could prove or disprove the model.

Here an oscillation is only a model that requires the least number of parameters to describe the variation. This can be updated when more observations become available (line 460). 
Reviewer 2: A. The author does a good job at describing how the analysis was done, and providing literature references to place the work in context. However, I am concerned that the data presentation and logical arguments are quite lengthy and detailed, and are challenging to keep straight. To assist the wider audience, a few things could readily be done to enhance the text's accessibility:

This reviewer offered a number of excellent suggestions to make the manuscript better.

Reviewer 2: 2) The claim that the XFEL beam pumps the sample to $500 \mathrm{~K}$ relies on evidence that is quite indirect-Fig $6 i$ assumes that the synchrotron datasets at various thermometer temperatures can be plotted on a straight line with respect to the c3 coefficient, and that the XFEL data can then be placed directly on this line. It would be more cautious if the text admitted that there is no other physical evidence for this claim. In other words, this is an interesting but unique claim for which other evidence should be gathered.

This is a good suggestion. A note has been added to state that the temperature jump is solely based on a linear extrapolation (lines 525-527).

Reviewer 2: B. The subject matter and techniques are extremely specialized, and I believe that more must be done to help the audience place the methods and claims into a larger context. Four specific concerns are:

1) Even for an expert protein crystallographer, the spin states of CO-myoglobin may not be common knowledge. It is important to give enough basic chemistry background in the Introduction so the reader does not erroneously conclude that the data actually distinguish between high spin and low spin electrons. Rather, we know fundamentally that in $\mathrm{MbFe}(\mathrm{II}) \mathrm{CO}, \mathrm{Fe}(\mathrm{II})$ has 6 electrons, all are paired so the total spin is $\mathrm{S}=0$. This is because the $5 d$-orbitals are split by the ligand field into 3 lower $t 2 g+2$ upper eg; so the lower orbitals are all full. On photolysis of $C O$, the ligand field and orbital splitting decrease, so there are four unpaired electrons with total spin $S=2$. Including this textbook material would help to capture a larger audience.

This is an excellent point. I have incorporated a little more into the brief background of spin crossover in Introduction (lines 108-116). 
Reviewer 2: 1) There are exactly 11 novel claims made in Fig. 1, represented by blue lines in the time chart with corresponding text description. It would be good for each of these blue lines to indicate which Figures contain the supporting evidence. For example, the "heme heating up to 500-600K" is clearly a consequence of Fig. 6i. Cross-referencing in this manner would help the reader keep track of the logical arguments.

2) A similar comment applies to the claims in the Introduction. Clearly line 172 "high spin iron" follows from Fig. 3; however, this reviewer cannot locate the evidence for "high frequency vibrational mode" and "low frequency oscillations" in lines 178-179.

\section{I have implemented these cross referencing.}

Reviewer 2: 3) Part of the problem is that the reader does not necessarily have the information about what is "high frequency" and "low frequency" in a particular context; after all, the paper discusses 18-orders of magnitude of time in Fig. 1. It seems safer to avoid the use of "high" and "low" and possibly give specific numerical ranges or functional descriptions.

I have added the corresponding wavenumbers where high or low frequency is mentioned.

Reviewer 2: 4) Also the line 213-214 claim that an oscillation occurs more in the solvent channels than in the protein or heme is similarly difficult to find in the visual evidence. Is it within Figure 6 somewhere? One possible strategy would be to take specific claims out of the Introduction since they are repeated anyway in the sequence of Results.

Fig. 7 is about the oscillation largely in the solvent. Cross referencing has been added.

Reviewer 2: Various typographical and language corrections were found:

Line 56 "of hundreds of $K$ "

Line 80 "first few"

Line 151 "preemptively avoiding"

Line 192 "not sufficient time points"

Line 204 please specify what kind of shift 
bioRxiv preprint doi: https://doi.org/10.1101/634071; this version posted July 12,2019 . The copyright holder for this preprint (which was not certified by peer review) is the author/funder. All rights reserved. No reuse allowed without permission.

\section{Line 577 "features can be found" \\ Line 608 "structural" \\ Line 630 "conceals" \\ Line 929 "first few"}

All fixed. 


\section{Reviewers' comments:}

Reviewer \#1 (Remarks to the Author):

The author challenges an ambitious task to extract tiny structural information possessing $\mathrm{x}$-ray scattering data sets of a resolution only about $1.8 \AA$ while usually this requires data of much higher resolution, atomic 1.0-1.2 $\AA$ or even ultrahigh, about $0.8 \AA$. Creation of such tools would be a great step in development of X-ray research methods. Unfortunately, the results presented do not allow one to agree unconditionally with the author's conclusions about the progress achieved, as well as with the interpretation of the results obtained when processing previously published experimental data. In particular, the annotated author's conclusions on the visualization of the $3 \mathrm{~d}$ orbitals and structural rearrangements in the spin crossover process raise significant doubts. An essential drawback of the suggested approach is the stage of the "manual" rotation of the found set of basic density distributions with unclear criteria of the best choice. This makes it difficult to reproduce the presented results. The suggestions and findings of the author could be a basis for further discussions, but the categorical style of interpretation of the results does not correspond to the level of evidence of these conclusions and may give rise to false illusions for non-experts in $x-$ ray data processing. All this requires a substantial revision of the manuscript and of the conception.

A key problem in identifying small changes in the electron density of an object in a series of experiments under variable conditions is that one never has the true distribution of electron density. What a researcher has instead is a Fourier synthesis of electron density, always of a limited resolution. Saying nothing about experimental errors, these syntheses contain larger or smaller distortion, caused by lack of the necessary data. The magnitude of such distortions, called Fourier series truncation errors, can be significant and these distortions can mask the subtle changes in the electron density. In particular, this leads to the fact that individual electrons and hydrogen atoms become distinguishable in Fourier difference syntheses only when working with data sets at resolution of $1.0-1.2 \AA$ (atomic resolution), while partial charges (density of valence electrons) become visible only at about $0.8 \AA$ (ultra-high resolution) and only under certain restrictions on the structure refinement protocol (Afonine et al., 2004, Acta Cryst. D60, 260-274). Even 0.6 resolution syntheses can contain Fourier series truncation errors exceeding the signal level and mimicking structural details. The results presented by the author have been obtained at resolution of $1.8 \AA$, which is intermediate between "medium" and "high" resolutions according to the existing crystallographic classification. At such conditions, sufficiently large distortions of Fourier syntheses are expected. In this regard, the questions arise: "What are the changes studied in the manuscript? Are they those in the electronic structure of the sample or just a game of the Fourier series truncation errors?" The author does not pay any attention to these circumstances. Moreover, the author regularly speaks of "ultrahigh resolution", which 
can confuse the reader.

The author's approach to the study of a series of experimental electron density distributions close to each other is based on a representation of each of these distributions as a weighted sum of a fixed set of density distributions pairwise uncorrelated, considered as a set of basis vectors mutually orthogonal. A visual study of the evolution of electron density under changing of external conditions is substituted by a numeric analysis of the weights with which these basis functions enter into one or another experimental density distribution. Sets of basic vectors can be selected in many ways and the basic distributions themselves may have no structural meaning. The recommendation of the author is to choose the basis in which "... an appropriate perspective can be found to elucidate the relationship between the core data and metadata clearly and concisely". This recommendation is extremely vague, opening up the scope for the selection of the "perspective" that confirms most the hypothesis of the researcher, and makes it difficult to reproduce and validate the results obtained.

The start data is a set of several series of difference maps. The first series is based on the differences between results of the "dark" (without the use of exciting light pulses) experiment and ones in which the crystals were irradiated both light X-ray pulses ("light" sets), with different time delay

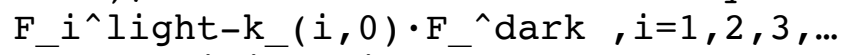
The remaining difference maps were calculated using only "light" data sets $F_{-} i^{\wedge} l i g h t-k_{-}(i, j) \cdot F_{-} j^{\wedge} l i g h t, i=j, j+1, \ldots$ Here $k_{-}(i, j)$ are scäling factors, individual for each map.

I start with a naive crystallographic explanation of the results. The first series of maps is shown in Fig. S2. These maps do not reproduce the typical pattern of difference maps, but rather reproduce a flipped (the one with the sign changed) image of the electron density distribution in the vicinity of the heme (the initial distribution is shown in Fig. S5). This is apparently caused by a imbalance in the difference syntheses of the first series, i.e. by too large values of the coefficients $k_{-}(i, 0)$, that results in the $-F^{\wedge}$ dark component dominating the maps. After decomposition, a balanced differential density distribution appears as the basic U1 distribution (Fig. S3). This map is close to the difference map of $1.8 \AA$ resolution. The excessive presence of the term $-F^{\wedge}$ dark in the initial difference syntheses $\mathrm{F}^{\wedge}$ light- $[\mathrm{k} \cdot \mathrm{F} \rrbracket \wedge$ dark is compensated by $\mathrm{U} 4$ component (Fig. $3 c)$, which resembles again the flipped image of density in the vicinity of the heme (Fig. S5). As noted by the author, in light-tolight difference syntheses $F_{-} i^{\wedge} l i g h t-k \cdot F_{-} j^{\wedge} l i g h t$ the $U 4$ component is absent. This can be explained by a better balance of syntheses coefficients when working with data sets obtained under the same experimental conditions (in the presence of both "light" and "dark" $\mathrm{x}-$ ray pulses) and, as a result, the absence of a "ghost" corresponding to the initial heme density distribution. 
The author suggests a totally different explanation declaring U4 component as visualization of $3 \mathrm{~d}$ orbital. An attention is not paid to the similarity of this distribution with the density distributions in the vicinity of the heme, shown in Fig. S2 and S5, as well as to the discrepancy between the size of the orbitals and the size of the density fragments in the U4 component noted by the author. Similarly, the author ignores that, when an electron transfers from one orbital to another, the image of the orbital in a difference map should appear twice: as a negative density of the orbital that has lost an electron, and a positive density of the orbitals that have gained an electron. He does not mention either the question on a principal possibility to see such a subtle change in $1.8 \AA$ resolution maps. The absence of the U4 component in the light-light series of difference maps is presented as evidence of the speed of this transition.

By this particular example, I would like to illustrate that the results obtained allow different interpretations that could be a subject of discussion, and that the author's interpretation is rather vague and should not be presented as a proof.

Vladimir Y. Lunin

Reviewer \#2 (Remarks to the Author):

Investigating the light-triggered release of $\mathrm{CO}$ from myoglobin, the author collectively re-examines electron density maps from room temperature and cryo-trapped structures, as well as the time-dependent XFEL paper [Barends et al. (2015)] covering the -0.1 ps to 150 ps time evolution. Mutual difference maps and single-dataset omit maps are analyzed with the Schmidt et al. (2003) method of singular value decomposition (SVD), to identify features in the electron density that change with either time, sample, or temperature. This reviewer does not know if SVD has ever been applied over so many independent variables, and if so it is worth mentioning.

The analysis leads to several astonishing claims highly relevant to myoglobin/CO binding, including that 1) the high-spin 3d orbitals can be visualized throughout the time series, 2) the XFEL pulse heats up the specimen to $\sim 500 \mathrm{~K}$, and 3 ) there is an oscillation with a 220 ps period.

A. The author does a good job at describing how the analysis was done, and providing literature references to place the work in context. However, I am concerned that the data presentation and logical arguments are quite lengthy and detailed, and are challenging to keep straight. To assist the wider audience, a few things could readily be done to enhance the text's accessibility:

1) There are exactly 11 novel claims made in Fig. 1, represented by blue lines in the time chart with corresponding text description. It would be good for each of these blue lines to indicate which Figures 
contain the supporting evidence. For example, the "heme heating up to 500-600K" is clearly a consequence of Fig. 6i. Cross-referencing in this manner would help the reader keep track of the logical arguments.

2) A similar comment applies to the claims in the Introduction. Clearly line 172 "high spin iron" follows from Fig. 3; however, this reviewer cannot locate the evidence for "high frequency vibrational mode" and "low frequency oscillations" in lines 178-179.

3) Part of the problem is that the reader does not necessarily have the information about what is "high frequency" and "low frequency" in a particular context; after all, the paper discusses 18-orders of magnitude of time in Fig. 1. It seems safer to avoid the use of "high" and "low" and possibly give specific numerical ranges or functional descriptions.

4) Also the line 213-214 claim that an oscillation occurs more in the solvent channels than in the protein or heme is similarly difficult to find in the visual evidence. Is it within Figure 6 somewhere? One possible strategy would be to take specific claims out of the Introduction since they are repeated anyway in the sequence of Results.

B. The subject matter and techniques are extremely specialized, and I believe that more must be done to help the audience place the methods and claims into a larger context. Four specific concerns are:

1) Even for an expert protein crystallographer, the spin states of COmyoglobin may not be common knowledge. It is important to give enough basic chemistry background in the Introduction so the reader does not erroneously conclude that the data actually distinguish between high spin and low spin electrons. Rather, we know fundamentally that in $\mathrm{MbFe}$ (II)CO, Fe(II) has 6 electrons, all are paired so the total spin is $\mathrm{S}=0$. This is because the 5 d-orbitals are split by the ligand field into 3 lower $t 2 g+2$ upper eg; so the lower orbitals are all full. On photolysis of $\mathrm{CO}$, the ligand field and orbital splitting decrease, so there are four unpaired electrons with total spin $\mathrm{S}=2$. Including this textbook material would help to capture a larger audience.

2) The author is completely correct (lines 237-239) to describe the literature-reported electron densities with unique shapes as being directly influenced by electrons occupying specific orbitals. However, there is a very large difference between the current diffraction dataset that diffracts to 1.8 Angstrom resolution, and the literature report of Hirano et al. (2016), showing iron 3d and sulfur 3p density at 0.48 Angstrom resolution. It is important to specifically compare the numerical resolution limits for the Hirano and the present work at both lines 236 and 370 ; in order to fully communicate that the present work is not simply a routine report of orbital density. Rather, the current work is lower-resolution and more speculative in nature.

3) It is further stated (lines 390-393) that normal 2Fo-Fc maps cannot reveal small orbital changes, but that SVD analysis is highly 
sensitive. If true, this would be a critical factor, and everyone should be using SVD to analyze structure comparisons. It would be highly beneficial to lay out the evidence and controls, or provide literature references.

4) The left-singular vectors are not unique-this is correctly described in the supplementary material starting at line 1201. At 1249 it is stated that a Givens rotation is applied so that an appropriate perspective can be found to elucidate the relationship between the core data and metadata. However, this leaves us with a situation where the main results of the paper (e.g., the U4 difference map showing high-spin density) are not based on a deterministic procedure! To paraphrase what I understand from this description, U4 was chosen by manually finding a Givens rotation that most clearly correlates with time-dependence. However, the author does not give a protocol to find that rotation, nor a metric to judge when the rotation is optimized. If I were to perform an SVD analysis on a new protein system, how would I go about applying the Ren methodology to this new system? The core issue here is reproducibility-it would be good if more details were given about how the rotations were derived, for this particular work. Would the conclusions have been robust if slightly different Uvectors were chosen?

C. Finally, some aspects of the central results could be presented with greater caution, considering that they are somewhat speculative, and that they depend on interpretation.

1) The author explains that the initial report of the observation of 3d orbitals (Zuo el al 1999) was somewhat controversial. In particular Scerri (2000) states that orbitals of single electrons are fundamentally not observable, and recommends that observations be described in terms of electron density. This would be the most cautious language. However, haven't the philosophical arguments now run their course after 20 years, so it should now be safe to speak of the U4 component as revealing the high-spin orbitals? Unfortunately, the author's own description shows that the electron density is only marginally interpretable as a 3d orbital. Line 368 states that the 3d orbital for an isolated $\mathrm{Fe}$ (II) has 0.36 Angstrom radius, and line 369 that the orbital density in an Fe4S4 cluster has a maximum radius of 1 Angstrom. Meanwhile, the data shown here place the electron density at 1.7 to 1.9 Angstrom from the iron, depending on which map is considered, so it appears that this analysis is "first of its kind" result, rather than the observation of a $3 d$ orbital by established methods. It is somewhat difficult to understand the author's point of view in the following text (lines 372-394). It is stated that the cubic density is not the probability distribution of a $3 \mathrm{~d}$ electron, yet the paper states in many places that we observe a high-spin $3 d$ orbital.

2 ) The claim that the XFEL beam pumps the sample to $500 \mathrm{~K}$ relies on evidence that is quite indirect-Fig $6 i$ assumes that the synchrotron datasets at various thermometer temperatures can be plotted on a straight line with respect to the c3 coefficient, and that the XFEL 
data can then be placed directly on this line. It would be more cautious if the text admitted that there is no other physical evidence for this claim. In other words, this is an interesting but unique claim for which other evidence should be gathered.

3) It is difficult to understand why the time evolution in Fig. $2 \mathrm{f}$ is described as an "oscillation". Not even one full cycle is observed, so it should not be assumed to be a circular progression. If there were more time points available they could prove or disprove the model.

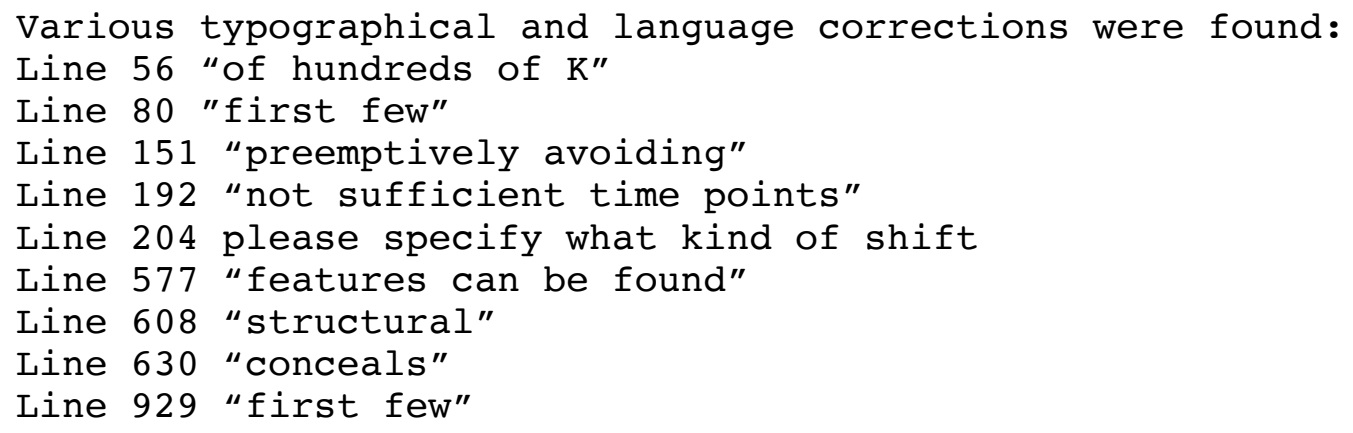

